\title{
Bow varieties and ALF spaces
}

$\operatorname{AUTHOR}(\mathrm{S}):$

TAKAYAMA, YUUYA

CITATION:

TAKAYAMA, YUUYA. Bow varieties and ALF spaces. Mathematical

Proceedings of the Cambridge Philosophical Society 2014, 158(01): 37 -

82

ISSUE DATE:

2014-12-11

URL:

http://hdl.handle.net/2433/198621

\section{RIGHT:}

(C) Cambridge Philosophical Society 2014; This is not the published version. Please cite only the published version.; この論文は出版社版で ありません。引用の際には出版社版をご確認ご利用ください。 


\title{
Bow varieties and ALF spaces
}

\author{
By Yuuya Takayama \\ Research Institute for Mathematical Sciences, Kyoto University \\ e-mail: takayama@kurims.kyoto-u.ac.jp
}

(Received )

Abstract

We introduce bow varieties and construct some ALF spaces as bow varieties.

\section{Introduction}

It is conjectured that noncompact hyper-Kähler 4-manifolds whose curvatures are in $L^{2}$ can be classified into 4 types - ALE, ALF, ALG and ALH - by their volume growth rates. ALE spaces satisfy $\operatorname{Vol}\left(B_{r}\right) \sim c r^{4}$, and it is known that their metrics converge asymptotically to the Euclidean metric at infinity. In 1989, Kronheimer classified and constructed ALE spaces [Kr2], and in 1990, Kronheimer and Nakajima described instantons on them $[\mathbf{K N}]$. It is worth noting that all ALE spaces and the moduli spaces of instantons are examples of quiver varieties introduced later by [Na1].

ALF spaces satisfy $\operatorname{Vol}\left(B_{r}\right) \sim c r^{3}$, and it is known that $S^{1} \times \mathbb{R}^{3}$ and Taub-NUT space are ALF, but their classification is not known. In 2010, Cherkis constructed some ALF spaces and instantons on them as bow varieties [C2]. Bow varieties are made up by a combination of quiver varieties and the moduli spaces of the solutions of Nahm's equations. In particular, bow varieties are constructed as infinite dimensional hyperKähler quotients, while quiver varieties are finite dimensional quotients.

In this paper, we study Cherkis' constructions of ALF spaces as bow varieties from a different point of view. Since hyper-Kähler quotients depend on parameters, Cherkis' bow varieties also depend on such parameters. First we conjecture that when all parameters are 0 , these bow varieties are isometric to Taub-NUT $/ \Gamma$ for some finite groups $\Gamma$. Indeed Kronheimer's 0-parameter hyper-Kähler quotients are isometric to $\mathbb{C}^{2} / \Gamma$. To prove this conjecture is the first step to establish that Cherkis' bow varieties are ALF. On the other hand, Taub-NUT/ $\Gamma$ is obtained by taking fixed points of a symmetric product of Taub-NUT space by the $\Gamma$-action. Thus, as hyper-Kähler quotients, we try to construct a symmetric product of Taub-NUT space and its fixed points by the $\Gamma$-action. And then we compare our hyper-Kähler quotients with Cherkis' bow varieties, and prove that Cherkis' bow varieties are ALF when their parameters are 0 .

We first introduce elemental bow varieties as basic building blocks, and study their properties. For example, we show that elemental bow varieties are isomorphic to certain quiver varieties as algebraic varieties, and are isomorphic to symmetric products of TaubNUT space as hyper-Kähler manifolds. Notice that it is not clear that infinite dimensional hyper-Kähler quotients are algebraic. We overcome this difficulty by using Kronheimer's theorem $[\mathbf{K r} \mathbf{1}]$ stating that the moduli space of the solutions of Nahm's equations on a 


\section{YUUYA TAKAYAMA}

closed interval is holomorphic symplectomorphic to $T^{*} G L(n, \mathbb{C})$. Then we can use a wellknown relation between hyper-Kähler quotients and geometric invariant theory (GIT) in a finite dimensional setting.

Next we construct some ALF spaces by using elemental bow varieties. We prove that the automorphism group of Taub-NUT space as hyper-Kähler manifolds preserving the origin is $\mathbb{Z}_{2} \ltimes S^{1}$. Therefore only type $A_{n}$ or $D_{n}$ group acts on Taub-NUT space among the $A D E$ classification of finite subgroups $\Gamma$ of $S U(2)$. Then taking fixed points of elemental bow varieties by the $\Gamma$-action, we obtain Taub-NUT/ $\Gamma$. Furthermore, by using the McKay correspondence, Taub-NUT/ $\Gamma$ can be constructed as bow varieties which associates with $A_{n}$-type bow (6.1) or $D_{n}$-type bow (7.1). We study the properties of these bow varieties. These things are summarised as follows:

TheOREM 0.1. Taub-NUT/An and their resolutions of singularities are constructed as $A_{n}$-type bow varieties and $A L F$. Taub-NUT/ $D_{n}$ are constructed as $D_{n}$-type bow varieties and $A L F$.

For $A_{n}$-type bow varieties, this result is well-known as mentioned in [C2]. $D_{n}$-type bow varieties we obtain in this paper is a little bit different from that of Cherkis. Details of this point are written in Remark 7.2 (iii). And the resolutions of singularities of Taub-NUT $/ D_{n}$ are also constructed as $D_{n}$-type bow varieties but we could not prove they are ALF.

This paper is organised as follows. In $\S 1$, we recall the definition of hyper-Kähler quotients and GIT quotients. In particular, we take care to deal with them in infinite dimensional setting. Since Nahm's equations are ordinary differential equations, we state analytical facts about them.

In $\S 2$, we define and study Nahm's equations. It is known that the moduli space of the solutions of Nahm's equations is regarded as a hyper-Kähler quotient $\mathcal{M}_{\mathrm{hK}}:=\mu_{I}^{-1}(0) \cap$ $\mu_{J}^{-1}(0) \cap \mu_{K}^{-1}(0) / \mathcal{G}$. And in order to research the relation between $\mathcal{M}_{\mathrm{hK}}$ and $T^{*} G L$, we consider another space $\mathcal{M}_{\text {sy }}:=\mu_{\mathbb{C}}^{-1}(0) / \mathcal{G}^{\mathbb{C}}$, where $\mu_{\mathbb{R}}=\mu_{I}$ and $\mu_{\mathbb{C}}=\mu_{J}+\sqrt{-1} \mu_{K}$. Then we study three spaces $\mathcal{M}_{\mathrm{hK}}, \mathcal{M}_{\mathrm{sy}}$ and $T^{*} G L$, and maps between them precisely.

In $\S 3$, we show the properties of the maps and give a proof of Kronheimer's theorem, for completeness.

In $\S 4$, we define elemental bow varieties. By their construction, elemental bow varieties have hyper-Kähler structures. We first prove elemental bow varieties are actually algebraic varieties by using Kronheimer's theorem. Moreover, we consider the relation between elemental bow varieties and particular quiver varieties, and prove elemental bow varieties are isomorphic to symmetric products of Taub-NUT space.

In $\S 5$, we calculate automorphisms of Taub-NUT preserving the origin. Then by the result of $\S 4$, they induce the action on elemental bow varieties. In this situation, finite group actions on elemental bow varieties are classified into $A_{n}$-type and $D_{n}$-type. For $\Gamma=A_{n}$ or $D_{n}$ we take the $\Gamma$-fixed points of elemental bow varieties. Then we have "Dynkin bows" in the same way as Kronheimer $[\mathbf{K r 2}]$. And we can obtain $A_{n}$-type and $D_{n}$-type ALF spaces but cannot obtain $E_{n}$-type ALF spaces in this way by the classification.

In $\S 6$, we study $A_{n}$-type bow varieties more precisely. These varieties are not elemental bow varieties, but the above arguments still work and have the same properties as elemental bow varieties.

In $\S 7$, we study $D_{n}$-type bow varieties in the same way as $\S 6$. 


\section{Preliminaries}

The moduli space of the solutions of Nahm's equations is regarded as a hyper-Kähler quotient. Since the acting group is infinite dimensional and noncompact, we carefully recall what properties of the group action guarantee the quotient to be a smooth manifold.

Then we review GIT quotients, which are used to study quiver varieties. And last we recall facts of ordinary differential equations we will use in this paper.

\subsection{Hyper-Kähler quotients}

We first recall definitions of hyper-Kähler manifolds and hyper-Kähler quotients with action of a (not necessary compact) Lie group.

Definition 1.1. ( $M, g, I, J, K)$ is called a hyper-Kähler manifold, if $(M, g)$ is a Riemann manifold and equipped with three complex structures $I, J$ and $K$ which satisfies the equations

$$
\nabla I=\nabla J=\nabla K=0 \text { and } I J=K,
$$

where $\nabla$ is the Levi-Civita connection with respect to the metric $g$.

Let $G$ be a Lie group acting smoothly on $M$ so as to preserve the metric $g$ and complex structures $I, J$ and $K$. A map $\mu=\left(\mu_{I}, \mu_{J}, \mu_{K}\right): M \rightarrow \mathfrak{g}^{*} \otimes \mathbb{R}^{3}$ is called a hyper-Kähler moment map if it satisfies

$$
\begin{aligned}
\left\langle d \mu_{A}(v), \xi\right\rangle & =g(A \tilde{\xi}, v), \quad v \in T M, \xi \in \mathfrak{g}, A=I, J, K, \\
\mu_{A}(g \cdot x) & =\operatorname{Ad}_{g}^{*} \mu_{A}(x), \quad x \in M, g \in G, A=I, J, K,
\end{aligned}
$$

where $\mathfrak{g}^{*}$ is the dual space of $\mathfrak{g}, \mathrm{Ad}^{*}: \mathfrak{g}^{*} \rightarrow \mathfrak{g}^{*}$ is the coadjoint map, $\langle$,$\rangle denotes the dual$ pairing between $\mathfrak{g}$ and $\mathfrak{g}^{*}$, and $\tilde{\xi}$ denotes a vector field induced by $\xi \in \mathfrak{g}$.

Put $Z=\left\{\zeta \in \mathfrak{g}^{*} \otimes \mathbb{R}^{3} \mid \operatorname{Ad}_{g}^{*}(\zeta)=\zeta\right.$ for all $\left.g \in G\right\}$ and take $\zeta \in Z$. In general, a hyper-Kähler moment map is not unique, because when $\mu$ is a hyper-Kähler moment map then $\mu+\zeta$ is also. Afterward we fix one of these hyper-Kähler moment maps and describe it as $\mu$ when we consider a hyper-Kähler moment map. Therefore a quotient space $\mu^{-1}(\zeta) / G$ is called a hyper-Kähler quotient. Considering the quotient, we assume the following conditions are satisfied:

Condition 1.2 .

(1) $\Gamma_{(M, G)}:=\{(x, g x) \in M \times M \mid x \in M, g \in G\}$ is closed in $M \times M$,

(2) $\gamma_{(M, G)}$ is a homeomorphism from $M \times G$ onto $\Gamma$, where $\gamma(x, g)=(x, g x)$,

(3) $G$ acts freely on $\mu^{-1}(\zeta)$.

The first condition is equivalent to that the quotient space is a Hausdorff, and the second and third give a structure of a smooth manifold on the quotient $[\mathbf{V}, \S 2.9]$. The second condition is equivalent to the properness of $\gamma$ because of the smoothness of the action, the Hausdorffness of $M$ and $G$, and the third condition. Once the quotient becomes a manifold, the argument in $[\mathbf{H K L R}]$ or $[\mathbf{G N}]$ works, and it has a structure of a hyperKähler manifold. These things are summarised as follows:

Proposition 1.3. If Condition 1.2 is satisfied, a hyper-Kähler quotient $\mu^{-1}(\zeta) / G$ is a (smooth) hyper-Kähler manifold. 
Therefore we will check these three conditions for Nahm's equations in $\S 2.1$.

REMARK 1.4. The third condition is necessary to be smooth, so if $G$ does not act freely on $p \in \mu^{-1}(\zeta)$, the quotient space $\mu^{-1}(\zeta) / G$ has a singular point at $G \cdot p$.

\subsection{GIT quotients and analytic stability}

There exists a way to treat quotient spaces in the algebraic geometry, it is called geometric invariant theory (GIT). First we review the way to construct quotient spaces in GIT, and next we mention the relation between GIT quotients and Kähler quotients.

\subsubsection{GIT quotients}

Let $X$ be an affine variety over $\mathbb{C}$ and $G^{\mathbb{C}}$ be a reductive algebraic group acting on $X$. An affine algebro-geometric quotient $X / / G^{\mathbb{C}}$ is defined as $\operatorname{Spec}\left(A(X)^{G^{\mathbb{C}}}\right)$, where $A(X)$ is the coordinate ring of $X$ and $A(X)^{G^{\mathbb{C}}}$ is the ring of invariants by the $G^{\mathbb{C}}$-action. By the theorem of Nagata, $A(X)^{G^{\mathbb{C}}}$ is a finitely generated algebra, so Spec is defined. In this situation, the following theorem is well-known (see $[$ MFK]).

TheOREM 1.5. (1) There exists a surjective morphism

$$
\phi: X \rightarrow X / / G^{\mathbb{C}}
$$

induced by the inclusion $A(X)^{G^{\mathbb{C}}} \subset A(X)$. Moreover, $\phi(x)=\phi(y)$ if and only if

$$
x \sim y \Leftrightarrow \overline{G^{\mathbb{C}} \cdot x} \cap \overline{G^{\mathbb{C}} \cdot y} \neq \emptyset,
$$

where "—" denotes the closure.

(2) The underlying space of $X / / G^{\mathbb{C}}$ is the set of closed $G^{\mathbb{C}}$-orbits modulo the equivalence relation defined by $x \sim y$ if and only if above relation holds.

By this theorem, we consider the closed orbits only. We say $x \in X$ is stable if $G^{\mathbb{C}} \cdot x$ is closed and its stabiliser is finite. Then $X / / G^{\mathbb{C}}$ is $X^{s} / \sim$, where $X^{s}$ denotes the set of stable points in $X$.

The idea of the affine algebro-geometric quotient can be modified. Let $\chi: G \rightarrow U(1)$ be a character, and $\chi$ also denotes its complexification,

$$
\chi: G^{\mathbb{C}} \rightarrow \mathbb{C}^{*} .
$$

Consider the trivial line bundle $X \times \mathbb{C}$ over $X$. Using $\chi$, we lift the $G^{\mathbb{C}}$-action to $X \times \mathbb{C}$ by

$$
g \cdot(x, z)=\left(g \cdot x, \chi(g)^{-1} z\right) \text { for }(x, z) \in X \times \mathbb{C} .
$$

Let $A(X)^{G^{\mathbb{C}}}, \chi^{n}$ be the space of polynomials satisfying $f(g \cdot x)=\chi(g)^{n} f(x)$. It can be identified with the space of $G^{\mathbb{C}}$-invariant sections of the above line bundle. If we set $\tilde{f}(x, z)=f(x) z^{n}, \tilde{f}$ is an element of $A(X \times \mathbb{C})^{G^{\mathbb{C}}}$. Then the direct sum

$$
\bigoplus_{n \geq 0} A(X)^{G^{\mathbb{C}}, \chi^{n}}
$$

is a finitely generated graded algebra. Hence we can define

$$
X / / \chi G^{\mathbb{C}}:=\operatorname{Proj}\left(\bigoplus_{n \geq 0} A(X)^{G^{\mathbb{C}}, \chi^{n}}\right) .
$$


This is called a geometric invariant theory quotient. The inclusion $A(X)^{G^{\mathbb{C}}} \oplus \oplus A(X)^{G^{\mathbb{C}}, \chi^{n}}$ induces a projective morphism

$$
X / /{ }_{\chi} G^{\mathbb{C}} \rightarrow X / / G^{\mathbb{C}}
$$

We say $x \in X$ is $\chi$-semistable if there exists $f \in A(X)^{G^{\mathbb{C}}}, \chi^{n}$ with $n \geq 1$ such that $f(x) \neq 0$. This happens if and only if the closure of $G^{\mathbb{C}}(x, z)$ does not intersect with $X \times\{0\}$ for $z \neq 0$. Let $X^{s s}(\chi)$ be the set of $\chi$-semistable points. We introduce an equivalence relation $\sim$ on $X^{s s}(\chi)$ by defining $x \sim y$ if and only if $\overline{G^{\mathbb{C}} \cdot x}$ and $\overline{G^{\mathbb{C}} \cdot y}$ intersects in $X^{s s}(\chi)$. The quotient space $X^{s s}(\chi) / \sim$ is bijective to the set of orbits $G^{\mathbb{C}} \cdot x$ such that $G^{\mathbb{C}} \cdot(x, z)$ is closed for $z \neq 0$. Then $X / / \chi G^{\mathbb{C}}$ is $X^{s s}(\chi) / \sim$.

\section{1·2·2. Relation between GIT quotients and Kähler quotients}

Here we show the relation in the same way as [Na2, Proposition 3.21]. A line bundle plays a main role in GIT, so we also need to consider a line bundle on a Kähler quotient.

Let $(M, \omega)$ be a Kähler manifold and $\pi: L \rightarrow M$ be a holomorphic hermitian line bundle. If its curvature form coincides with the Kähler form $\omega$, the pair $(M, L)$ is called a prequantisation of the Kähler manifold $M$. Explicitly, a function $h: L \rightarrow \mathbb{R}^{+}$is defined by the hermitian structure:

$$
h\left(z_{p}\right):=\left\|z_{p}\right\|_{p}^{2},
$$

where $z_{p} \in \pi^{-1}(p)$ and $\|\cdot\|_{p}$ is the hermitian norm. Then the connection form $\alpha$ of $L$ is defined as

$$
\alpha=\frac{1}{\pi \sqrt{-1}}(\partial-\bar{\partial})(\log h)=d^{c} \log h,
$$

and the curvature form is defined as the exterior derivative of $\alpha$ :

$$
d \alpha=\frac{1}{2 \pi \sqrt{-1}} \bar{\partial} \partial \log h=d d^{c} \log h .
$$

The definition of the prequantised Kähler manifold needs $\omega$ coincides with $s^{*}(d \alpha)$, where $s: M \rightarrow L$ is the 0 -section. We assume a compact Lie group $G$ acts on $M$.

Definition 1.9. A linearisation of the $G^{\mathbb{C}}$-action on $M$ is a holomorphic action of $G^{\mathbb{C}}$ on $L$ covering the action on $M$, and such that $G$ acts unitarily on the fibres.

We consider a Kähler quotient $\mu^{-1}(0) / G$. Then the following proposition is well-known.

Proposition 1.10. (Bryan $[\mathbf{B r}]$ ) A choice of a linearisation uniquely determines a moment map and conversely a choice of a moment map uniquely determines a linearisation.

In this situation, we say a point $p \in M$ is analytically semistable for a linearised action of $G^{\mathbb{C}}$ on $M$ if for every non-zero $z_{p} \in L_{p}$ a function $g \mapsto h\left(g \cdot z_{p}\right)$ is proper as a function on $G^{\mathbb{C}} / G_{p}^{\mathbb{C}}$. Here $G_{p}^{\mathbb{C}}$ denotes the stabiliser of $p$. We describe the set of analytically semistable points of $M$ as $M^{\text {ass }}$.

LEMma 1.11. (Bryan $[\mathbf{B r}])$ An orbit $G^{\mathbb{C}} \cdot p \subset M$ is analytically semistable if and only if $G^{\mathbb{C}} \cdot p$ meets $\mu=0$. Furthermore the set $G^{\mathbb{C}} \cdot p \cap \mu^{-1}(0)$ consists of exactly one $G$-orbit.

Thus we have a biholomorphism $\mu^{-1}(0) / G \cong M^{\text {ass }} / G^{\mathbb{C}} \cdot M^{\text {ass }} / G^{\mathbb{C}}$ is similar to the GIT 


\section{YUUYA TAKAYAMA}

quotient $M / / G^{\mathbb{C}}$, so we want to know their relation. Since the author does not know a general theory about this, we consider it in particular cases later.

For a later purpose, we quote the way how a moment map is determined by a linearisation from Bryan. For $\xi \in \mathfrak{g}$ we describe the induced vector field on $M$ as $\tilde{\xi}$ and the induced vector field on $L$ as $\tilde{\tilde{\xi}}$, that is, $\pi_{*} \tilde{\tilde{\xi}}=\tilde{\xi}$. Put $\mu_{\xi}=\langle\mu, \xi\rangle$. Define $\mu$ by the equation $\mu_{\xi}=s^{*}\left(\iota \tilde{\xi}^{\alpha)}\right.$. Then we have

$$
\begin{aligned}
g(I \tilde{\xi},-)=\iota_{\tilde{\xi}} \omega & =s^{*}\left(\iota_{\tilde{\xi}} d \alpha\right) \\
& =s^{*}\left(d_{\tilde{\tilde{\xi}}} \alpha+\mathfrak{L}_{\tilde{\xi}} \alpha\right)=d \mu_{\xi},
\end{aligned}
$$

where $\mathfrak{L}_{\tilde{\tilde{\xi}}} \alpha=0$ since $G$ acts unitarily on the fibres. Thus $\mu$ satisfies $d \mu_{\xi}=\iota_{\tilde{\xi}} \omega$. And we have

$$
\begin{aligned}
\mu_{[a, \xi]} & =s^{*}\left(\iota_{\widetilde{[a, \xi]}}^{\underset{\sim}{\sim}} \alpha\right)=s^{*}\left(\iota_{[\tilde{\tilde{\xi}}, \tilde{\tilde{a}}]} \alpha\right) \\
& =-s^{*}\left(\mathfrak{L}_{\tilde{\tilde{a}}} \iota \tilde{\tilde{\xi}} \alpha-\iota_{\tilde{\xi}} \mathfrak{\mathfrak { L } _ { \tilde { a } }} \alpha\right) \\
& =-\mathfrak{L}_{\tilde{\tilde{a}}} \mu_{\xi} .
\end{aligned}
$$

This implies $\mu(g \cdot x)=\operatorname{Ad}_{g}^{*} \mu(x)$, thus $\mu$ defines a moment map.

In this paper, we describe a point $p \cdot G$ of a quotient space $M / G$ as $[p]_{G}$.

\section{Nahm's equations and three spaces}

In this section we prepare to prove Kronheimer's theorem. Therefore we endow three spaces $\mathcal{M}_{\mathrm{hK}}, \mathcal{M}_{\text {sy }}$ and $T^{*} G L$ with $\mathcal{C}^{\infty}$ structures, integrable almost complex structures, and symplectic structures. And next we define maps between them.

\subsection{Nahm's equations and space $\mathcal{M}_{h K}$}

We define the space $\mathcal{M}_{\mathrm{hK}}$ as the moduli space of the solutions of Nahm's equations. We start from giving the definition of Nahm's equations.

Let $\mathcal{I}=[0,1]$ be an interval parameterised by $s$, and $L_{1}^{2}(\mathcal{I}, \mathfrak{u}(n))$ be a set of $\mathfrak{u}(n)$ valued $L_{1}^{2}$ functions on $\mathcal{I}$. We describe the norm of $\mathfrak{u}(n)$ as $\|U\|^{2}=\operatorname{tr} U U^{*}$ and the norm of $L_{1}^{2}(\mathcal{I}, \mathfrak{u}(n))$ as $\|T\|_{L_{1}^{2}}^{2}=\int\|T(s)\|^{2} d s+\int\|d T / d s\|^{2} d s$. Put $\mathcal{H}=\left\{\left(T_{0}, T_{1}, T_{2}, T_{3}\right) \in\right.$ $\left.L_{1}^{2}(\mathcal{I}, \mathfrak{u}(n))^{\oplus 4}\right\}$. We give a flat metric on $\mathcal{H}: d s^{2}=\int_{0}^{1} d T_{0} d T_{0}^{*}+d T_{1} d T_{1}^{*}+d T_{2} d T_{2}^{*}+$ $d T_{3} d T_{3}^{*} d s$, i.e. for $u=\left(\tau_{0}, \tau_{1}, \tau_{2}, \tau_{3}\right), u^{\prime}=\left(\tau^{\prime}{ }_{0}, \tau^{\prime}{ }_{1}, \tau^{\prime}{ }_{2}, \tau^{\prime}{ }_{3}\right) \in T \mathcal{H}$,

$$
d s^{2}\left(u, u^{\prime}\right)=\int_{0}^{1} \operatorname{tr}\left(\tau_{0}{\tau^{\prime}}_{0}^{*}+\tau_{1}{\tau^{\prime}}_{1}^{*}+\tau_{2} \tau_{2}^{\prime *}+\tau_{3} \tau_{3}^{\prime *}\right) d s .
$$

We define complex structures on $\mathcal{H}$ by

$$
\begin{aligned}
I:\left(\tau_{0}, \tau_{1}, \tau_{2}, \tau_{3}\right) & \mapsto\left(-\tau_{1}, \tau_{0},-\tau_{3}, \tau_{2}\right), \\
J:\left(\tau_{0}, \tau_{1}, \tau_{2}, \tau_{3}\right) & \mapsto\left(-\tau_{2}^{*}, \tau_{3}^{*}, \tau_{0}^{*},-\tau_{1}^{*}\right), \\
& =\left(\tau_{2},-\tau_{3},-\tau_{0}, \tau_{1}\right), \\
K:\left(\tau_{0}, \tau_{1}, \tau_{2}, \tau_{3}\right) & \mapsto\left(\tau_{3}, \tau_{2},-\tau_{1},-\tau_{0}\right) .
\end{aligned}
$$

A group $\mathcal{G}_{00}:=\left\{u \in L_{2}^{2}(\mathcal{I}, U(n)) \mid u(0)=u(1)=\mathrm{id}\right\}$ acts on $\mathcal{H}$ as follows:

$$
\begin{aligned}
& u \cdot T_{0}=u T_{0} u^{-1}-\frac{d u}{d s} u^{-1}, \\
& u \cdot T_{k}=u T_{k} u^{-1}, \quad k=1,2,3 .
\end{aligned}
$$


This action preserves the metric and the complex structures.

REMARK 2.3. On the interval $\mathcal{I}$, there is a compact inclusion $L_{1}^{2} \hookrightarrow \mathcal{C}^{0}$. Hence a map $f(s, u)=T_{0}(s) u$ satisfies the Lipschitz condition

$$
\left\|T_{0}(s) u-T_{0}(s) u^{\prime}\right\|_{\infty} \leq \sup _{s}\left\|T_{0}(s)\right\|_{\infty}\left\|u-u^{\prime}\right\|_{\infty},
$$

where $\sup \left\|T_{0}\right\|$ is independent of $s, u$ and $u^{\prime}$. So we can solve the equation $u \cdot T_{0}=$ 0 (i.e. $\frac{d}{d s} u=T_{0} u$ ) with respect to a given initial value of $u$ at $s=0$. In other words, any $\left(T_{0}, T_{1}, T_{2}, T_{3}\right)$ can be sent to $\left(0, T^{\prime}{ }_{1}, T^{\prime}{ }_{2}, T^{\prime}{ }_{3}\right)$ by an element of $\mathcal{G}_{0 *}=\left\{u \in L_{2}^{2}(\mathcal{I}, U(n)) \mid\right.$ $u(0)=\mathrm{id}\}$.

DEFINITION 2.4. We consider the following ordinary differential equations (Nahm's equations)

$$
\left\{\begin{array}{l}
\frac{d}{d s} T_{1}+\left[T_{0}, T_{1}\right]+\left[T_{2}, T_{3}\right]=0 \\
\frac{d}{d s} T_{2}+\left[T_{0}, T_{2}\right]+\left[T_{3}, T_{1}\right]=0 \\
\frac{d}{d s} T_{3}+\left[T_{0}, T_{3}\right]+\left[T_{1}, T_{2}\right]=0 .
\end{array}\right.
$$

We denote left hand sides by $\mu_{I}, \mu_{J}$ and $\mu_{K}$ respectively.

These equations are preserved by the $\mathcal{G}_{00}$-action, and $\mu=\left(\mu_{I}, \mu_{J}, \mu_{K}\right)$ is a hyper-Kähler moment map. In fact, for example with respect to $I$, we have

$$
\begin{aligned}
\left\langle d \mu_{I}(u), \xi\right\rangle & =\int_{0}^{1} \operatorname{tr} d\left\{\frac{d T_{1}}{d s} \xi^{*}+\left[T_{0}, T_{1}\right] \xi^{*}+\left[T_{2}, T_{3}\right] \xi^{*}\right\}\left(\tau_{0}, \tau_{1}, \tau_{2}, \tau_{3}\right) d s \\
& =\int_{0}^{1} \operatorname{tr}\left\{-\frac{d \tau_{1}}{d s} \xi-\left[\tau_{0}, T_{1}\right] \xi-\left[T_{0}, \tau_{1}\right] \xi-\left[\tau_{2}, T_{3}\right] \xi-\left[T_{2}, \tau_{3}\right] \xi\right\} d s \\
& =\left[\operatorname{tr}\left\{-\tau_{1} \xi\right\}\right]_{0}^{1}+\int_{0}^{1} \operatorname{tr}\left\{\tau_{1} \frac{d}{d s} \xi-\left[\tau_{0}, T_{1}\right] \xi-\left[T_{0}, \tau_{1}\right] \xi-\left[\tau_{2}, T_{3}\right] \xi-\left[T_{2}, \tau_{3}\right] \xi\right\} d s \\
& =\int_{0}^{1} \operatorname{tr}\left\{-\left(\left[\xi, T_{1}\right]\right) \tau_{0}^{*}+\left(\left[\xi, T_{0}\right]-\frac{d}{d s} \xi\right) \tau_{1}^{*}+\left(\left[\xi, T_{3}\right]\right) \tau_{2}^{*}+\left(\left[\xi, T_{2}\right]\right) \tau_{3}^{*}\right\} d s \\
& =g(I \tilde{\xi}, u),
\end{aligned}
$$

where we used integration by parts in the third equality. Therefore, $\mathcal{M}_{\mathrm{hK}}$ is defined as a hyper-Kähler quotient.

Remark 2.5. Put $\mathcal{G}_{* *}=L_{2}^{2}(\mathcal{I}, U(n))$. $\mathcal{G}_{* *}$ acts on $\mathcal{H}$ in the same way as $\mathcal{G}_{00}$. In this case, the hyper-Kähler moment map is replaced by $\mu_{A}+\delta_{0} T_{A}(0)-\delta_{1} T_{A}(1)$ for $A=I, J, K$. This is because for $\xi \in$ Lie $\mathcal{G}_{* *}$ we have

$$
\begin{aligned}
{\left[\operatorname{tr}\left\{-\tau_{1} \xi\right\}\right]_{0}^{1} } & =\operatorname{tr}\left\{-\tau_{1}(1) \xi(1)+\tau_{1}(0) \xi(0)\right\} \\
& =\left\langle\delta_{1} d T_{1}(1)\left(\tau_{1}\right)-\delta_{0} d T_{1}(0)\left(\tau_{1}\right), \xi\right\rangle .
\end{aligned}
$$

And when we change coordinate $T_{A}(s) \mapsto T_{A}(s)+f_{A}(s)$ id by $f_{A} \in L_{1}^{2}(\mathcal{I}, \sqrt{-1} \mathbb{R})$, we have

$$
\mu_{A}+\delta_{0} T_{A}(0)-\delta_{1} T_{A}(1) \mapsto \mu_{A}+\frac{d f_{A}}{d s} \mathrm{id}+\delta_{0}\left(T_{A}(0)+f_{A}(0) \mathrm{id}\right)-\delta_{1}\left(T_{A}(1)+f_{A}(1) \mathrm{id}\right)
$$

In order to see $\mathcal{M}_{\mathrm{hK}}$ is a hyper-Kähler manifold, we check Condition 1.2. 
Lemma 2.6. $\Gamma_{\left(\mathcal{H}, \mathcal{G}_{00}\right)}=\left\{(x, g x) \in \mathcal{H} \times \mathcal{H} \mid x \in \mathcal{H}, g \in \mathcal{G}_{00}\right\}$ is closed in $\mathcal{H} \times \mathcal{H}$.

Proof. Let $\left\{\left(T_{0}^{n}, T_{k}^{n}\right),\left(T^{\prime n}, T^{\prime \prime}{ }_{k}^{n}\right)\right\}(k=1,2,3)$ be a convergent sequence in $\mathcal{H} \times \mathcal{H}$, where $T^{\prime \prime}{ }_{0}^{n}=u^{n} \cdot T_{0}, T_{k}^{\prime n}=u^{n} \cdot T_{k}$. Put

$$
\lim _{n \rightarrow \infty}\left\{\left(T_{0}^{n}, T_{k}^{n}\right),\left({T^{\prime}}_{0}^{n}, T^{\prime n}{ }_{k}\right)\right\}=\left\{\left(T_{0}^{\infty}, T_{k}^{\infty}\right),\left(T_{0}^{\prime \infty}, T_{k}^{\prime \infty}\right)\right\} .
$$

We define $v^{n}=u^{n} /\left\|u^{n}\right\|_{L_{2}^{2}}$, then we have $\left\|v^{n}\right\|_{L_{2}^{2}}=1$ for all $n$. We may assume $v^{n}$ converges in the $\mathcal{C}^{1}$ sense by taking a subsequence, because $L_{2}^{2}$ is embedded compactly in $\mathcal{C}^{1}$ on $\mathcal{I}$. Hence both sides of the equations

$$
\begin{aligned}
v^{n} T_{0}^{n}-\frac{d}{d s} v^{n} & =T_{0}^{\prime n} v^{n}, \\
v^{n} T_{k}^{n} & =T^{\prime \prime}{ }_{k}^{n} v^{n}, \quad k=1,2,3
\end{aligned}
$$

converge as $n \rightarrow \infty$, at least in the $\mathcal{C}^{0}$ sense:

$$
\begin{aligned}
v^{\infty} T_{0}^{\infty}-\frac{d}{d s} v^{\infty} & ={T^{\prime}}_{0}^{\infty} v^{\infty}, \\
v^{\infty} T_{k}^{\infty} & ={T^{\prime}}_{k}^{\infty} v^{\infty}, \quad k=1,2,3 .
\end{aligned}
$$

We have to prove that $\left({T^{\prime}}_{0}^{\infty}, T^{\prime}{ }_{k}^{\infty}\right)$ is in the $\left(T_{0}^{\infty}, T_{k}^{\infty}\right)$-orbit.

First we suppose $\lim \left\|u^{n}\right\|=\infty$. According to $v^{n}(0)=u^{n}(0) /\left\|u^{n}\right\|=\mathrm{id} /\left\|u^{n}\right\|$, we have $v^{\infty}(0)=\mathrm{id} / \lim \left\|u^{n}\right\|$. Hence, $v^{\infty}(0)$ is 0 . By $(2.7), v^{\infty}$ is 0 for any $s$, so we can put $\sup \left\|v^{n}\right\|, \sup \left\|d v^{n} / d s\right\|<\varepsilon$ for enough large $n$. We obtain

$$
\begin{aligned}
\left\|v^{n}-v^{\infty}\right\|_{L_{2}^{2}}^{2} & =\left\|v^{n}\right\|_{L_{1}^{2}}^{2}+\left\|\frac{d^{2}}{d s^{2}} v^{n}\right\|_{L^{2}}^{2} \\
& \leq \sup _{s}\left(\left\|v^{n}(s)\right\|^{2}+\left\|\frac{d}{d s} v^{n}(s)\right\|^{2}\right)+\left\|\frac{d}{d s}\left(v^{n} T_{0}^{n}-T_{0}^{\prime n} v^{n}\right)\right\|^{2} \\
& \leq \varepsilon+\sup _{s}\left\|\left(\frac{d v^{n}}{d s} T_{0}^{n}\right)^{2}+\left(v^{n} \frac{d}{d s} T_{0}^{n}\right)^{2}+\left(\frac{d v^{n}}{d s} T_{0}^{\prime n}\right)^{2}+\left(v^{n} \frac{d}{d s} T_{0}^{\prime n}\right)^{2}\right\| \\
& \leq \varepsilon\left\{1+\sup \left\|T_{0}^{n}+\frac{d}{d s} T_{0}^{n}+T_{0}^{\prime n}+\frac{d}{d s} T_{0}^{\prime n}\right\|\right\},
\end{aligned}
$$

so $v^{n}$ converges to $v^{\infty}=0$ in the $L_{2}^{2}$ sense. This contradicts with $\left\|v^{\infty}\right\|_{L_{2}^{2}}=\lim \left\|v^{n}\right\|_{L_{2}^{2}}=1$.

Next we suppose $\lim \left\|u^{n}\right\|=c\left(\geq 1=\left\|u^{n}(0)\right\|\right)$. In this case, we solve (2.7) with $v^{\infty}(0)=\mathrm{id} / c$. We claim that the solution $v^{\infty}(s)$ is nondegenerate for any $s$. If so, $c v^{\infty}$ enters in $\mathcal{G}_{00}$, and hence $\left(T_{0}^{\prime \infty}, T_{k}^{\prime \infty}\right)$ is in the $\left(T_{0}^{\infty}, T_{k}^{\infty}\right)$-orbit as required.

We prove the claim. Let $A(s), B(s)$ be a solution of the following equations:

$$
\begin{aligned}
& \frac{d}{d s} A(s)=-T_{0}^{\prime \infty}(s) A(s), \quad A(0)=\mathrm{id}, \\
& \frac{d}{d s} B(s)=B(s) T_{0}^{\infty}(s), \quad B(0)=\mathrm{id} .
\end{aligned}
$$

Then the solution $v^{\infty}(s)$ of $(2.7)$ can be described as $A(s) v^{\infty}(0) B(s)$, and for all $s$ we get $\operatorname{det} A(s) \neq 0$ and $\operatorname{det} B(s) \neq 0$. This is because of the following argument. Put $A(s)=\left(a_{1}(s), a_{2}(s), \ldots, a_{n}(s)\right)$, where $a_{i}(s) \mathrm{s}$ are column vectors and satisfy

$$
\frac{d}{d s} a_{i}(s)=-T_{0}^{\prime \infty}(s) a_{i}(s), \quad a_{i}(0)=\delta_{i j} .
$$

If $\operatorname{det} A(s)=0$ for some $s$, we have $\sum_{i} k_{i} a_{i}(s)=0, k_{i} \in \mathbb{C}$ with $\left(k_{i}\right) \in \mathbb{C}^{n} \backslash 0$. From $(2.8)$ 
we have

$$
\frac{d}{d s}\left(\sum_{i} k_{i} a_{i}(s)\right)=-T_{0}^{\prime \infty}(s)\left(\sum_{i} k_{i} a_{i}(s)\right),
$$

hence $\sum_{i} k_{i} a_{i}(s)=0$ becomes a solution for any $s$ by the uniqueness of solutions. This contradicts with $A(0)=$ id.

We check the remaining two conditions.

Proposition 2.9. The moduli space of the solutions of Nahm's equations is a hyperKähler manifold.

Proof. We check the third of Condition 1.2. Assume $u \cdot T_{0}=T_{0}$. There exists $u_{0} \in \mathcal{G}_{0 *}$ such that $u_{0} \cdot 0=T_{0}$ by Remark 2.3. Then we have $\left(u_{0}^{-1} u u_{0}\right) \cdot 0=0$ i.e. $\frac{d}{d s}\left(u_{0}^{-1} u u_{0}\right)=0$ by the assumption. This implies $u=\mathrm{id}$, because of $\left(u_{0}^{-1} u u_{0}\right)(s)=\left(u_{0}^{-1} u u_{0}\right)(0)=\mathrm{id}$.

Let us check the second condition. When an orbit $\left(T_{0}^{\prime n}, T_{k}^{\prime n}\right)=\left(u^{n} \cdot T_{0}, u^{n} \cdot T_{k}\right)$ converges to $\left(T^{\prime}{ }_{0}^{\infty}, T^{\prime}{ }_{k}^{\infty}\right)$, then $u^{n}$ converges to $u^{\infty}$ (by taking a subsequence) from the proof of Lemma 2.6. This means $\gamma$ is proper.

We describe the tangent space of this manifold in order to write down complex structures and Kähler structures of $\mathcal{M}_{\mathrm{hK}}$. Let $\left(\tau_{0}, \tau_{1}, \tau_{2}, \tau_{3}\right)$ be an element of the tangent space of $\mathcal{M}_{\mathrm{hK}}$ at $\left[\left(T_{0}, T_{1}, T_{2}, T_{3}\right)\right]_{\mathcal{G}_{00}}$, where $\left[\left(T_{0}, T_{1}, T_{2}, T_{3}\right)\right]_{\mathcal{G}_{00}}$ denotes a point represented by $\left(T_{0}, T_{1}, T_{2}, T_{3}\right) \in \mathcal{H}$. We regard $\tau_{k}$ as an element in $\left.L_{1}^{2}(\mathcal{I}, \mathfrak{u}(n))\right)$. In general, a tangent space of a hyper-Kähler quotient is given by $\operatorname{Ker} d \mu_{I} \cap \operatorname{Ker} d \mu_{J} \cap \operatorname{Ker} d \mu_{K} \cap \operatorname{Im} \iota^{\perp}$, where $\iota$ is the differential of the group action. And $\perp$ is the orthogonal complement by the induced Kähler metric. From the results of calculation, Ker $d \mu_{I}$ is given by $\frac{d}{d s} \tau_{1}+\left[T_{0}, \tau_{1}\right]-$ $\left[T_{1}, \tau_{0}\right]+\left[T_{2}, \tau_{3}\right]-\left[T_{3}, \tau_{2}\right]=0$ and $\operatorname{Im} \iota$ is given by $\left\{\left(\left[h, T_{0}\right]-\frac{d}{d s} h,\left[h, T_{1}\right],\left[h, T_{2}\right],\left[h, T_{3}\right]\right) \in\right.$ $T \mathcal{M}_{\mathrm{hK}} \mid h \in$ Lie $\left.\mathcal{G}_{00}\right\}$. Hence, $\left(\tau_{0}, \tau_{1}, \tau_{2}, \tau_{3}\right) \in T_{\left[\left(T_{0}, T_{1}, T_{2}, T_{3}\right)\right]} \mathcal{M}_{\mathrm{hK}}$ satisfies the following equations:

$$
\left\{\begin{array}{l}
\frac{d}{d s} \tau_{0}+\left[T_{0}, \tau_{0}\right]+\left[T_{1}, \tau_{1}\right]+\left[T_{2}, \tau_{2}\right]+\left[T_{3}, \tau_{3}\right]=0 \\
\frac{d}{d s} \tau_{1}+\left[T_{0}, \tau_{1}\right]-\left[T_{1}, \tau_{0}\right]+\left[T_{2}, \tau_{3}\right]-\left[T_{3}, \tau_{2}\right]=0 \\
\frac{d}{d s} \tau_{2}+\left[T_{0}, \tau_{2}\right]-\left[T_{1}, \tau_{3}\right]-\left[T_{2}, \tau_{0}\right]+\left[T_{3}, \tau_{1}\right]=0 \\
\frac{d}{d s} \tau_{3}+\left[T_{0}, \tau_{3}\right]+\left[T_{1}, \tau_{2}\right]-\left[T_{2}, \tau_{1}\right]-\left[T_{3}, \tau_{0}\right]=0 .
\end{array}\right.
$$

These linear equations can be solved with respect to a given initial value. Thus the dimension of the solution space of these equations is $2 n^{2}$, and this means the dimension of $\mathcal{M}_{\mathrm{hK}}$ is also $2 n^{2}$. The metric and complex structures are induced by (2.1) and (2.2), and they are described as the restriction of (2.1) and (2.2) to $T \mathcal{M}_{\mathrm{hK}}$. Note that three Kähler forms are described as follows:

$$
\begin{aligned}
& \omega_{I}=\int_{0}^{1} \operatorname{tr}\left\{-d T_{0} \wedge d T_{1}-d T_{2} \wedge d T_{3}\right\} d s \\
& \omega_{J}=\int_{0}^{1} \operatorname{tr}\left\{d T_{0} \wedge d T_{2}-d T_{1} \wedge d T_{3}\right\} d s \\
& \omega_{K}=\int_{0}^{1} \operatorname{tr}\left\{d T_{0} \wedge d T_{3}+d T_{1} \wedge d T_{2}\right\} d s .
\end{aligned}
$$

Here $\omega_{J}+\sqrt{-1} \omega_{K}$ is a holomorphic symplectic form with respect to $I$. 


\section{YUUYA TAKAYAMA}

\subsection{Space $\mathcal{M}_{\text {sy }}$}

We construct the second space $\mathcal{M}_{\mathrm{sy}}=\mu_{\mathbb{C}}^{-1}(0) / \mathcal{G}^{\mathbb{C}}$ in this subsection. We use the same space $\mathcal{H}$ as in the last subsection. We focus on the complex structure $I$, we put

$$
\alpha:=\frac{1}{2}\left(T_{0}+\sqrt{-1} T_{1}\right), \quad \beta:=\frac{1}{2}\left(T_{2}+\sqrt{-1} T_{3}\right),
$$

so $\alpha$ and $\beta$ are $\mathfrak{g l}(n)$-valued $L_{1}^{2}$ functions. By using them, Nahm's equations can be described as

$$
\begin{aligned}
\frac{d}{d s} \beta+2[\alpha, \beta] & =0 \text { (The "complex equation"), } \\
\hat{F}(\alpha, \beta):=\frac{d}{d s}\left(\alpha+\alpha^{*}\right)+2\left(\left[\alpha, \alpha^{*}\right]+\left[\beta, \beta^{*}\right]\right) & =0 \text { (The "real equation"). }
\end{aligned}
$$

The complex equation and the real equation correspond to $\mu_{J}+\sqrt{-1} \mu_{K}=0$ and $\mu_{I}=0$ respectively. We put $\mu_{\mathbb{C}}=\mu_{J}+\sqrt{-1} \mu_{K}$ and $\mu_{\mathbb{R}}=\mu_{I}$. We introduce the complexification of $\mathcal{G}_{00}: \mathcal{G}_{00}^{\mathbb{C}}=\left\{g \in L_{2}^{2}(\mathcal{I}, G L(n)) \mid g(0)=g(1)=\right.$ id $\}$. It acts on $\mathcal{H}$ as follows:

$$
\begin{aligned}
& g \cdot \alpha=g \alpha g^{-1}-\frac{1}{2} \frac{d g}{d s} g^{-1}, \\
& g \cdot \beta=g \beta g^{-1} .
\end{aligned}
$$

This action preserves only the complex equation. And it is clear that $\mu_{\mathbb{C}}$ is a moment map with respect to the symplectic form $\omega_{J}+\sqrt{-1} \omega_{K}$ and the $\mathcal{G}_{00}^{\mathbb{C}}$-action.

Therefore we define the space $\mathcal{M}_{\text {sy }}$ as the symplectic quotient $\mu_{\mathbb{C}}^{-1}(0) / \mathcal{G}_{00}^{\mathbb{C}}$. This space is also a manifold, by the same argument as in Lemma 2.6 and Proposition 2.9. Note that the compactness of the group $U(n)$ was not used in the proofs.

Let us consider the tangent space of this space and the complex structure. There is no metric preserved by the complex action, the tangent space is not defined as an orthogonal complement. Instead the tangent space of $\mathcal{M}_{\mathrm{sy}}$ is given as the middle cohomology of the following short exact sequence:

$$
\operatorname{Lie} \mathcal{G}_{00}^{\mathbb{C}} \stackrel{\iota_{C}}{\longrightarrow} L_{1}^{2}\left(\mathcal{I}, \mathfrak{g l}^{\oplus 2}\right) \stackrel{d \mu_{\mathbb{C}}}{\longrightarrow} \operatorname{Lie} \mathcal{G}_{00}^{\mathbb{C}},
$$

that is $T_{[(\alpha, \beta)]} \mathcal{M}_{\mathrm{sy}} \cong \operatorname{Ker}_{(\alpha, \beta)} d \mu_{\mathbb{C}} / \operatorname{Im}_{(\alpha, \beta)} \iota \mathbb{C}$. And Ker $d \mu_{\mathbb{C}}$ and $\operatorname{Im} \iota_{\mathbb{C}}$ are given respectively by

$$
\begin{gathered}
\left\{(A, B) \mid \frac{d}{d s} B+2[A, \beta]+2[\alpha, B]=0\right\}, \\
\left\{\left([h, \alpha]-\frac{1}{2} \frac{d}{d s} h,[h, \beta]\right) \mid h \in \operatorname{Lie} \mathcal{G}_{00}^{\mathbb{C}}\right\} .
\end{gathered}
$$

Put $\mathcal{G}_{* *}^{\mathbb{C}}=L_{2}^{2}(\mathcal{I}, G L(n)) . \mathcal{G}_{* *}^{\mathbb{C}}$ acts on $\mathcal{H}$ and preserves the complex equation similarly to $\mathcal{G}_{00}^{\mathbb{C}}$. Then $G L \times G L$ acts on $\mathcal{M}_{\text {sy }}$ because of the isomorphism between $\mathcal{G}_{* *}^{\mathbb{C}} / \mathcal{G}_{00}^{\mathbb{C}}$ and $G L \times G L$. The action induces an isomorphism between $T_{[(\alpha, \beta)]} \mathcal{M}_{\mathrm{sy}}$ and $T_{[([g] \cdot \alpha,[g] \cdot \beta)]} \mathcal{M}_{\mathrm{sy}}$. For Ker $d \mu_{\mathbb{C}}$ and $\operatorname{Im} \iota_{\mathbb{C}}$, this isomorphism can be written as follows:

$$
\begin{aligned}
{[g] \cdot(A, B) } & =\left(g A g^{-1}, g B g^{-1}\right), \\
{[g] \cdot\left([h, \alpha]-\frac{1}{2} \frac{d}{d s} h,[h, \beta]\right) } & =\left(\left[g h g^{-1}, g \cdot \alpha\right]-\frac{1}{2} \frac{d}{d s}\left(g h g^{-1}\right),\left[g h g^{-1}, g \cdot \beta\right]\right),
\end{aligned}
$$

where $[g]$ is an element of $G L \times G L$ represented by $g \in \mathcal{G}_{* *}^{\mathbb{C}}$. The symplectic form on $\mathcal{M}_{\text {sy }}$ 
is induced by the symplectic form $\omega=\omega_{J}+\sqrt{-1} \omega_{K}$ on $\mathcal{H}$. It can be described as

$$
\begin{aligned}
\omega\left((A, B),\left(A^{\prime}, B^{\prime}\right)\right) & :=4 \int_{0}^{1} \operatorname{tr}(d \alpha \wedge d \beta) d s\left((A, B),\left(A^{\prime}, B^{\prime}\right)\right) \\
& =4 \int_{0}^{1} \operatorname{tr}\left(A B^{\prime}-A^{\prime} B\right) d s .
\end{aligned}
$$

Let $\xi$ be an element of Lie $\mathcal{G}_{00}^{\mathbb{C}}$ and $v$ of Ker $d \mu_{\mathbb{C}}$, then we have

$$
\omega\left(\xi^{*}, v\right)=\left\langle d \mu_{\mathbb{C}}(v), \xi\right\rangle=0 .
$$

Thus the symplectic form $\omega$ is well-defined on $\operatorname{Ker} d \mu_{\mathbb{C}} / \operatorname{Im} \iota_{\mathbb{C}}$.

REMARK 2.11. The author does not know how to calculate the dimension of $\mathcal{M}_{s y}$ directly. It will be cleared up that it is also equal to $2 n^{2}$, according to the existence of a homeomorphism to $\mathcal{M}_{h K}$ or $T^{*} G L$.

\subsection{Space $T^{*} G L$}

A space $T^{*} G L$ is the cotangent bundle of the complex general linear group $G L(n, \mathbb{C})$. This is a complex manifold and an affine variety. The topology of this space is induced as a variety. Furthermore, as a cotangent bundle, $T^{*} G L$ has a symplectic structure. We define 2 -form $\Omega$ at $(u, \eta) \in T^{*} G L=G L \times \mathfrak{g}$ by

$$
\Omega\left((U, H),\left(U^{\prime}, H^{\prime}\right)\right)=-2 \operatorname{tr}\left\{\left(H U^{\prime}-H^{\prime} U\right)+\left[U, U^{\prime}\right] \eta\right\},
$$

where $(U, H) \in \mathfrak{g l}^{\oplus 2}$ is identified with a right invariant vector field $\left(\tilde{U}_{(u, \eta)}, \tilde{H}_{(u, \eta)}\right) \in$ $T_{(u, \eta)} T^{*} G L$.

LEMMA 2.13. $\Omega$ is a symplectic form on $T^{*} G L$.

Proof. Let $\pi: T^{*} G L \rightarrow G L$ be the projection. Then, in general, the fundamental form $\theta$ is defined as follows:

$$
\begin{aligned}
\theta_{(u, \eta)}((U, H)) & =-\eta(d \pi(U, H)) \\
& =-\operatorname{tr}\left(\eta d u u^{-1}\right)(U, H),
\end{aligned}
$$

where $d u u^{-1}$ is the Maurer-Cartan form of $G L$. Thus we have a symplectic form

$$
d \theta_{(u, \eta)}=-\operatorname{tr}\left(d \eta \wedge d u u^{-1}+\eta d u u^{-1} \wedge d u u^{-1}\right),
$$

and $2 d \theta$ is also a symplectic form.

\subsection{Maps between three spaces}

We define maps $F, F^{-1}, G$ and $G^{-1}$ in this subsection.

$$
\begin{aligned}
& \mu_{\mathbb{R}}^{-1}(0) \cap \underset{\mathbb{C}}{\mu_{\mathbb{C}}^{-1}(0) / \mathcal{G}_{00}} \underset{F^{-1}}{\longleftarrow} \mu_{\mathbb{C}}^{-1}(0) / \mathcal{G}_{00}^{\mathbb{C}} \underset{\Psi}{\longleftarrow} T_{\Psi}^{*} G L \\
& {\left[\left(T_{0}, T_{1}, T_{2}, T_{3}\right)\right]_{\mathcal{G}_{00}} \quad[(\alpha, \beta)]_{\mathcal{G}_{00}^{\complement}} \quad(u, \eta) .}
\end{aligned}
$$

\subsubsection{Maps $F$ and $F^{-1}$}

Both $\mu_{\mathbb{R}}$ and $\mu_{\mathbb{C}}$ are defined on $\mathcal{H}$, so the map $F$ can be defined as the inclusion map from $\mathcal{M}_{\mathrm{hK}}$ to $\mathcal{M}_{\mathrm{sy}}$ :

$$
F\left(\left[\left(T_{0}, T_{1}, T_{2}, T_{3}\right)\right]_{\mathcal{G}_{00}}\right)=\left[\left(\frac{1}{2}\left(T_{0}+\sqrt{-1} T_{1}\right), \frac{1}{2}\left(T_{2}+\sqrt{-1} T_{3}\right)\right)\right]_{\mathcal{G}_{00}^{\complement}} .
$$




\section{YUUYA TAKAYAMA}

In order to consider the inverse map of $F$, we solve the real equation $\mu_{\mathbb{R}}=0$.

Proposition 2.14. Let $(\alpha, \beta)$ be an element of $\mathcal{H}$ (not necessary of $\mu_{\mathbb{C}}^{-1}(0)$ ). There exists at least one $g \in \mathcal{G}_{00}^{\mathbb{C}}$ such that $g \cdot(\alpha, \beta) \in \mu_{\mathbb{R}}^{-1}(0)$.

Proof. The idea of this proof follows from $[\mathbf{K i}]$ and $[\mathbf{N a 1}]$. We consider the gradient flow of $f=\left\|\mu_{\mathbb{R}}\right\|^{2}$ starting from $(\alpha, \beta)$. The path is described as $g^{t} \cdot(\alpha, \beta)$ by using some $g^{t} \in \mathcal{G}_{00}^{\mathbb{C}}$. And according to Neeman $[\mathbf{N e}], g^{t} \cdot(\alpha, \beta)$ converges to $\left(\alpha^{\infty}, \beta^{\infty}\right)$. At this time the convergence of $g^{t}$ follows from Lemma 2.6.

Put $\mu_{\mathbb{R}}\left(\alpha^{\infty}, \beta^{\infty}\right)=\xi \in \operatorname{Lie} \mathcal{G}_{00}$. The limit $\left(\alpha^{\infty}, \beta^{\infty}\right)$ is a critical point of $f$, hence the vector field generated by $\xi$ vanishes at $\left(\alpha^{\infty}, \beta^{\infty}\right)$. The vanishing of the vector field can be described as

$$
\left[\xi, \alpha^{\infty}\right]-\frac{1}{2} \frac{d}{d s} \xi=0, \quad\left[\xi, \beta^{\infty}\right]=0 .
$$

But $\xi(0)=0$, so we have $\xi \equiv 0$ and $\mu_{\mathbb{R}}\left(\alpha^{\infty}, \beta^{\infty}\right)=0$. Then $g^{\infty}$ is the required element of $\mathcal{G}_{00}^{\mathbb{C}}$.

Theorem 2.15. (Donaldson [Do]) Furthermore, if $(\alpha, \beta)$ in $\mu_{\mathbb{C}}^{-1}(0), g \in \mathcal{G}_{00}^{\mathbb{C}}$ at Proposition 2.14 is unique up to $\mathcal{G}_{00}$.

Proof. We sketch the proof by Donaldson. In order to remove an ambiguity of the $\mathcal{G}_{00}$-action, put $h=g^{*} g \in L_{2}^{2}(\mathcal{I}, G L(n) / U(n))$. And define

$$
\Phi(h):=\log \max (\text { eigenvalue of } h) .
$$

Then according to [Do, Lemma 2.10], we have

$$
\begin{aligned}
\frac{d^{2}}{d s^{2}} \Phi(h) & \geq-2\left(\|\hat{F}(\alpha, \beta)\|+\left\|\hat{F}\left(\alpha^{\prime}, \beta^{\prime}\right)\right\|\right), \\
\frac{d^{2}}{d s^{2}} \Phi\left(h^{-1}\right) & \geq-2\left(\|\hat{F}(\alpha, \beta)\|+\left\|\hat{F}\left(\alpha^{\prime}, \beta^{\prime}\right)\right\|\right),
\end{aligned}
$$

where $\left(\alpha^{\prime}, \beta^{\prime}\right)=g \cdot(\alpha, \beta)$. Suppose both $(\alpha, \beta)$ and $\left(\alpha^{\prime}, \beta^{\prime}\right)$ satisfy the real equation, then the right hand sides of (2.16) are 0. This means that the maximal eigenvalue of $h$ is not larger than 1 on $\mathcal{I}$ and that the minimal eigenvalue of $h$ is not less than 1 on $\mathcal{I}$. They imply $h=$ id.

In this way, the map $F^{-1}$ is defined.

\subsubsection{Maps $G$ and $G^{-1}$}

By Remark 2.3, we can solve the equation

$$
g \cdot 0=-\frac{1}{2} \frac{d g}{d s} g^{-1}=\alpha, \quad g(0)=\mathrm{id} .
$$

When $g^{-1} \cdot \alpha=0, g^{-1} \cdot \beta$ becomes constant because of the complex equation. Therefore the solution $(\alpha, \beta)$ of the complex equation is sent to $(0, \eta)$, where $\eta$ is a constant $\beta(0)$, by the action of a group $\mathcal{G}_{0 *}^{\mathbb{C}}:=\left\{g \in L_{2}^{2}(\mathcal{I}, G L) \mid g(0)=\right.$ id $\}$. Since $\mathcal{G}_{0 *}^{\mathbb{C}} / \mathcal{G}_{00}^{\mathbb{C}} \cong G L(n)$, the map can be defined,

$$
G(\alpha, \beta):=(g(1), \eta),
$$

where $g$ is the solution of (2.17). 
Conversely, we define $G^{-1}$ as follows. Take $(u, \eta) \in G L \times \mathfrak{g l}$ and $g \in \mathcal{G}_{0 *}^{\mathbb{C}}$ which satisfy $g(0)=\operatorname{id}$ and $g(1)=u$. Then the definition of $G^{-1}$ is

$$
G^{-1}(u, \eta):=g \cdot(0, \eta)=\left(-\frac{1}{2} \frac{d g}{d s} g^{-1}, g \eta g^{-1}\right) .
$$

Needless to say, $[g \cdot(0, \eta)]_{\mathcal{G}_{00}^{\mathbb{C}}} \in \mu_{\mathbb{C}}^{-1}(0) / \mathcal{G}_{00}^{\mathbb{C}}$ is independent on the choice of $g \in \mathcal{G}_{0 *}^{\mathbb{C}}$.

\section{Detailed proof of Kronheimer's theorem}

By using the results in the last section, we give a detailed proof of Kronheimer's theorem.

Theorem 3.1. (Kronheimer $[\mathbf{K r} \mathbf{1}]) \mathcal{M}_{h K}$ is holomorphic symplectomorphic to the cotangent bundle of the general linear group $T^{*} G L(n, \mathbb{C})$.

And by this morphism, $T^{*} G L(n, \mathbb{C})$ can be regarded as a hyper-Kähler manifold.

First we show $\mathcal{M}_{\mathrm{hK}}, \mathcal{M}_{\mathrm{sy}}$ and $T^{*} G L$ are homeomorphic. To do this, we should pay attention to their topologies. Bijective maps are already obtained, so we prove their continuities.

Next we prove they are diffeomorphic, holomorphic and symplectomorphic. Since these properties are defined among their tangent bundles, we should calculate the differential of the maps, and check they preserve the structures of three manifolds.

\subsection{Homeomorphy}

The next proposition and lemma are essential.

Proposition 3.2. The maps Fand $G$ are bijective and continuous.

Proof. The bijectivities are already known. Since $F$ is the inclusion map, its continuity is clear. To show the continuity of $G$ at $\left(\alpha_{0}, \beta_{0}\right) \in \mu_{\mathbb{C}}^{-1}(0)$, we check the Lipschitz condition. Let $(\alpha, \beta)$ be in the $\delta$-neighborhood of $\left(\alpha_{0}, \beta_{0}\right)$. Since $L_{1}^{2}$ is compactly embedded in $\mathcal{C}^{0}$, it means $\sup _{s}\left\|\alpha(s)-\alpha_{0}(s)\right\| \leq \delta, \sup _{s}\left\|\beta(s)-\beta_{0}(s)\right\| \leq \delta$, and moreover

$$
\left\|\alpha(s)-\alpha_{0}(s)\right\|_{\infty} \leq \delta,\left\|\beta(s)-\beta_{0}(s)\right\|_{\infty} \leq \delta, \text { for any } s \in \mathcal{I},
$$

where $\|X\|_{\infty}=\max _{i, j}\left|X_{i j}\right|$ as before. Then we have the following inequality:

$$
\begin{aligned}
\left\|\alpha(s) g-\alpha(s) g^{\prime}\right\|_{\infty} & =\max _{i, j}\left|\left\{\alpha(s)\left(g-g^{\prime}\right)\right\}_{i j}\right| \\
& \leq \max \left|\alpha(s)_{i j}\right| \max \left|\left(g-g^{\prime}\right)_{i j}\right| \\
& \left.\leq \sup _{s}\left\|\alpha_{0}\right\|_{\infty}+\delta\right)\left\|g-g^{\prime}\right\|_{\infty} .
\end{aligned}
$$

sup $\left\|\alpha_{0}\right\|+\delta$ is independent of $\alpha$, so the Lipschitz condition is satisfied.

Lemma 3.3. The map $G \circ F$ is proper.

Proof. We have a trivial inequality:

$$
\left\|\prod_{i=1}^{k} A_{i}\right\| \leq n^{k} \prod_{i=1}^{k}\left\|A_{i}\right\|, \quad A_{i} \in G L(n) .
$$

This follows from the estimate of all the entries of $A_{i}$ by $\left\|A_{i}\right\|$.

The map $(G \circ F)^{-1}$ is given as follows. Take $(u, \eta) \in T^{*} G L$ and $\left(T_{0}, T_{1}, T_{2}, T_{3}\right)=(G \circ$ 


\section{YUUYA TAKAYAMA}

$F)^{-1}(u, \eta) \in \mu_{\mathbb{R}}^{-1}(0) \cap \mu_{\mathbb{C}}^{-1}(0) / \mathcal{G}_{00}$. In this situation, $\left(\left(T_{0}+\sqrt{-1} T_{1}\right) / 2,\left(T_{2}+\sqrt{-1} T_{3}\right) / 2\right)$ can be described as $g \cdot(0, \eta)$, where $g$ satisfies the differential equation,

$$
\frac{d}{d s}\left(h^{-1} \frac{d h}{d s}\right)+2\left[\eta, h^{-1} \eta^{*} h\right]=0, \text { where } h=g^{*} g, g(0)=\mathrm{id}, \text { and } g(1)=u^{-1} .
$$

The existence and uniqueness of the solution of this equation follows from Theorem 2.15. This equation is preserved by the $\mathcal{G}_{0 *}$-action, so we can assume $T_{0}=0$ from the beginning. Then in order to prove this lemma, first we show that $\left\|T_{1}(s)\right\|,\left\|T_{2}(s)\right\|$ and $\left\|T_{3}(s)\right\|$ are estimated by $\|u\|,\left\|u^{-1}\right\|$ and $\|\eta\|$ independent of $s$. Then from Nahm's equations we have

$$
\begin{aligned}
\left\|\frac{d}{d s} T_{1}\right\| & \leq\left\|\left[T_{2}, T_{3}\right]\right\| \leq 2 n^{2}\left\|T_{2}\right\|\left\|T_{3}\right\|, \\
\left\|\frac{d^{2}}{d s^{2}} T_{1}\right\| & \leq\left\|\left[\frac{d}{d s} T_{2}, T_{3}\right]\right\|+\left\|\left[T_{2}, \frac{d}{d s} T_{3}\right]\right\| \leq 4 n^{4}\left\|T_{1}\right\|\left(\left\|T_{2}\right\|^{2}+\left\|T_{3}\right\|^{3}\right),
\end{aligned}
$$

so the first and second derivatives are also estimated by $\|u\|,\left\|u^{-1}\right\|$ and $\|\eta\|$. Thus $T_{k}$ s are in $L_{2}^{\infty}$, and the compactness of the inclusion $L_{2}^{\infty} \hookrightarrow L_{1}^{2}$ implies that the subset $\left\{\left(\sqrt{-1} T_{1}, T_{2}+\sqrt{-1} T_{3}\right) \mid\right.$ estimated as above $\}$ is compact if the subset $\{(u, \eta)\}$ is compact.

From the relation between $T_{1}, T_{2}, T_{3}, h$ and $\eta$, we have inequalities

$$
\begin{gathered}
\left\|T_{1}\right\|^{2}=\operatorname{tr}\left(h^{-1} \frac{d h}{d s}\right)^{2} \leq\left\|h^{-1} \frac{d h}{d s}\right\|^{2}, \\
\left\|T_{2}+\sqrt{-1} T_{3}\right\|^{2}=\operatorname{tr}\left(\eta h^{-1} \eta^{*} h\right) \leq n^{4}\|\eta\|^{2} \sup _{s}\left\|h^{-1}\right\| \sup _{s}\|h\| .
\end{gathered}
$$

Therefore it is enough to estimate $h$ and $h^{-1}$. By Theorem 2.15, we have

$$
\frac{d^{2}}{d s^{2}} \Phi(h) \geq-2\left\|\left[\eta, \eta^{*}\right]\right\| \text {. }
$$

Put $f(s)=\Phi\left(\left(u u^{*}\right)^{-1}\right) s+\left\|\left[\eta, \eta^{*}\right]\right\| s(1-s)$, we get $\frac{d^{2}}{d s^{2}}(f-\Phi(h)) \leq 0$ and $f-\Phi(h)=0$ at $s=0,1$. Hence we have

$$
\Phi(h) \leq \Phi\left(\left(u u^{*}\right)^{-1}\right) s+\left\|\left[\eta, \eta^{*}\right]\right\| s(1-s) .
$$

In general, $\max \left(\right.$ eigenvalue of $\left.X X^{*}\right) \leq\|X\|^{2} \leq n \max \left(\right.$ eigenvalue of $\left.X X^{*}\right)$ holds, and since $h$ is a self-adjoint matrix, we have (the $i$-th eigenvalue of $\left.h h^{*}\right)=($ the $i$-th eigenvalue of $h)^{2}$. This leads to

$$
\begin{aligned}
\sup _{s}\|h\| & \leq \sqrt{n} \sup _{s} \exp \Phi(h) \\
& \leq \sqrt{n} \sup _{s}\left\{\exp \left(\Phi\left(\left(u u^{*}\right)^{-1}\right) s\right) \exp \left(\left\|\left[\eta, \eta^{*}\right]\right\| s(1-s)\right)\right\} \\
& \leq \sqrt{n}\left\|u^{-1}\right\|^{2} \exp \left(\frac{n^{2}}{2}\|\eta\|^{2}\right) .
\end{aligned}
$$

Similarly, we have $\sup _{s}\left\|h^{-1}\right\| \leq \sqrt{n}\|u\|^{2} \exp \left(\frac{n^{2}}{2}\|\eta\|^{2}\right)$. Then,

$$
\left\|T_{2}+\sqrt{-1} T_{3}\right\|^{2} \leq n^{5}\left\|u^{-1}\right\|^{2}\|u\|^{2}\|\eta\|^{2} e^{n^{2}\|\eta\|^{2}} .
$$

Integrating (3.4), we get

$$
\begin{aligned}
\left\|h^{-1} \frac{d h}{d s}-\left.h^{-1} \frac{d h}{d s}\right|_{s=0}\right\| & =\left\|-2 \int_{0}^{s}\left[\eta, h^{-1} \eta^{*} h\right] d s\right\| \\
& \leq 2 \int_{0}^{s}\left(\left\|\eta h^{-1} \eta^{*} h\right\|+\left\|h^{-1} \eta^{*} h \eta\right\|\right) d s .
\end{aligned}
$$


As a consequence, we have

$$
\begin{aligned}
\left\|h^{-1} \frac{d h}{d s}\right\| & \leq 4 n^{4}\|\eta\|^{2} \sup \|h\| \sup \left\|h^{-1}\right\|+\left\|\left.\frac{d h}{d s}\right|_{s=0}\right\| \\
& \leq 4 n^{5}\|\eta\|^{2}\left\|u^{-1}\right\|^{2}\|u\|^{2} e^{n^{2}\|\eta\|^{2}}+\left\|\left.\frac{d h}{d s}\right|_{s=0}\right\| .
\end{aligned}
$$

We estimate the last term. $d h /\left.d s\right|_{s=0}$ means $\lim _{s \rightarrow+0} \frac{1}{s}(h(s)-\mathrm{id})$, and we have the following inequality:

$$
\begin{aligned}
\|h-\mathrm{id}\|^{2} & \leq n(\exp \Phi(h)-1)^{2} \\
& \leq n\left\{\left(\Phi\left(\left(u u^{*}\right)^{-1}\right)+\left\|\left[\eta, \eta^{*}\right]\right\|\right) s+O\left(s^{2}\right)\right\}^{2} .
\end{aligned}
$$

Hence we get

$$
\begin{aligned}
\left\|\left.\frac{d h}{d s}\right|_{s=0}\right\| & \leq \lim _{s \rightarrow+0} \frac{\sqrt{n}}{s}\left\{\left(\log \left\|u^{-1}\right\|^{2}+\frac{n^{2}}{2}\|\eta\|^{2}\right) s+O\left(s^{2}\right)\right\} \\
& \leq \sqrt{n}\left(\log \left\|u^{-1}\right\|^{2}+\frac{n^{2}}{2}\|\eta\|^{2}\right) .
\end{aligned}
$$

Summarising them, we can conclude.

Proposition 3.5. The maps Fand $G$ are homeomorphic.

Proof. The continuities of $F$ and $G$ are by (3.2). From (3.3), $G \circ F$ is proper, so $G \circ F$ becomes homeomorphic. Then $F^{-1}=(G \circ F)^{-1} \circ G$ and $G^{-1}=F \circ(G \circ F)^{-1}$ are continuous.

\subsection{Other properties}

We consider the differential of $F$ and $G$, which are the maps between the tangent bundles of three spaces. Since $F$ is the inclusion map, $F_{*}$ is given by

$$
F_{*}\left(\tau_{0}, \tau_{1}, \tau_{2}, \tau_{3}\right)=\left[\left(\frac{1}{2}\left(\tau_{0}+\sqrt{-1} \tau_{1}\right), \frac{1}{2}\left(\tau_{2}+\sqrt{-1} \tau_{3}\right)\right)\right]_{\mathcal{G}_{00}^{\complement}},
$$

where $\left(\tau_{0}, \tau_{1}, \tau_{2}, \tau_{3}\right) \in T \mathcal{M}_{\mathrm{hK}}$ as $(2.10)$.

Let $\left(\alpha^{t}, \beta^{t}\right)$ be any path in $\mu_{\mathbb{C}}^{-1}(0)$ through $(\alpha, \beta)$ at $t=0$. Then the differential of $\left(\alpha^{t}, \beta^{t}\right)$ at $t=0$ gives $\left(\dot{\alpha}^{0}, \dot{\beta}^{0}\right) \in T_{(\alpha, \beta)} \mu_{\mathbb{C}}^{-1}(0)$. By definition, $G_{*}$ is given by the differential at $t=0$ of the solution of (2.17). So, we should solve

$$
\dot{\alpha}^{0}=-\frac{1}{2}\left(\frac{d \dot{g}^{0}}{d s} g^{-1}-\frac{d g}{d s} g^{-1} \dot{g}^{0} g^{-1}\right), \quad\left(\text { i.e. } \frac{d}{d s} \dot{g}^{0}+2 \dot{\alpha}^{0} g+2 \alpha \dot{g}^{0}=0\right),
$$

with $\dot{g}^{0}(0)=0$. Hence, by using the solution $\dot{g}^{0}$ of (3.6), we get

$$
G_{*}\left(\dot{\alpha}^{0}, \dot{\beta}^{0}\right)=\left(\dot{g}^{0}(1), \dot{\beta}^{0}(0)\right) .
$$

For a later purpose, we also calculate $G_{*}^{-1}$. Put $\left(u^{t}, \eta^{t}\right):=\left(u e^{t U}, \eta+t H\right),(H, U \in \mathfrak{g l})$. For $g \in \mathcal{G}_{0 *}^{\mathbb{C}}$ which satisfies $g(1)=u$, put $g^{t}=g e^{s t U}$. Then $g^{t}$ defines the map $G^{-1}$ for $\left(u^{t}, \eta^{t}\right)$ because $g^{t}$ satisfies $g^{t}(1)=u^{t}$. Then differentiating

$$
\begin{aligned}
\alpha^{t} & =-\frac{1}{2} \frac{d g^{t}}{d s} g^{t-1}, \\
\beta^{t} & =g^{t} \eta^{t} g^{t},
\end{aligned}
$$


at $t=0$, we find that

$$
G_{*}^{-1}(U, H)=\left(\dot{\alpha}^{0}, \dot{\beta}^{0}\right),
$$

where

$$
\begin{aligned}
\dot{\alpha}^{0} & =-\frac{1}{2} \frac{d \dot{g}^{0}}{d s} g^{-1}+\frac{1}{2} \frac{d g}{d s} g^{-1} \dot{g}^{0} g^{-1} \\
& =-\frac{1}{2} g U g^{-1}, \\
\dot{\beta}^{0} & =g \dot{\eta}^{0} g^{-1}+\dot{g}^{0} \eta g^{-1}-g \eta g^{-1} \dot{g}^{0} g^{-1} \\
& =g H g^{-1}+s g U \eta g^{-1}-s g \eta U g^{-1} .
\end{aligned}
$$

First, we show the next proposition by using these equations.

Proposition 3.8. The homeomorphisms $F$ and $G$ are diffeomorphisms.

Proof. In order to prove $F$ and $G$ are diffeomorphisms, we show $F_{*}$ and $G_{*}$ are isomorphisms between the tangent spaces.

First, we show $G_{*}$ is isomorphic. We take $g \in \mathcal{G}_{0 *}^{\mathbb{C}}$ which sends $(\alpha, \beta)$ to $(0, \eta)$, then $g$ induces an isomorphism from $T_{[(\alpha, \beta)]} \mathcal{M}_{\text {sy }}$ to $T_{[(0, \eta)]} \mathcal{M}_{\text {sy }}$. Hence it is enough to check $G_{*[(0, \eta)]}$ is isomorphic. Now we have

$$
\begin{aligned}
\operatorname{Ker}_{(0, \eta)} d \mu_{\mathbb{C}} & \cong\left\{(A, B) \mid \frac{d}{d s} B+2[A, \eta]=0\right\}, \\
\operatorname{Im}_{(0, \eta)} \iota \mathbb{C} & \cong\left\{\left(-\frac{1}{2} \frac{d}{d s} h,[h, \eta]\right) \mid h \in \operatorname{Lie} \mathcal{G}_{00}^{\mathbb{C}}\right\},
\end{aligned}
$$

and $G_{*[(0, \eta)]}: T_{[(0, \eta)]} \mathcal{M}_{\mathrm{sy}} \rightarrow T_{(\mathrm{id}, \eta)} T^{*} G L$ can be described as $(A(s), B(s)) \mapsto\left(\int_{0}^{1} A(s) d s\right.$, $B(0))$ from (3.6) and (3.7).

Suppose $G_{*[(0, \eta)]}(A, B)=(0,0)$, i.e. $\int_{0}^{1} A d s=0, B(0)=0$. Put $h(s):=-2 \int_{0}^{s} A\left(s^{\prime}\right) d s^{\prime}$, $h$ is in Lie $\mathcal{G}_{00}^{\mathbb{C}}$ because of $h(0)=h(1)=0$. Then we have

$$
\begin{aligned}
-\frac{1}{2} \frac{d}{d s} h & =A(s), \\
{[h, \eta] } & =-\int_{0}^{s} 2\left[A\left(s^{\prime}\right), \eta\right] d s^{\prime} \\
& =-\int_{0}^{s}\left(-\frac{d}{d s^{\prime}} B\right) d s^{\prime}=B(s),
\end{aligned}
$$

and hence $(A, B) \in \operatorname{Im} \iota_{\mathbb{C}}$. Conversely, for any $(U, H) \in T T^{*} G L$, the element $(U,-s[U, \eta]$ $+H)$ is sent to $(U, H)$. Therefore $G_{*}$ is isomorphic. (In fact, the dimensions of $\mathcal{M}_{\text {sy }}$ and $T^{*} G L$ are $2 n^{2}$, so the surjectivity follows from the injectivity.)

Next, we show $F_{*}$ is isomorphic. Suppose $\left[\left(\left(\tau_{0}+\sqrt{-1} \tau_{1}\right) / 2,\left(\tau_{2}+\sqrt{-1} \tau_{3}\right) / 2\right)\right]_{\mathcal{G}_{00}^{\mathbb{c}}}=0$ i.e. $\left(\left(\tau_{0}+\sqrt{-1} \tau_{1}\right) / 2,\left(\tau_{2}+\sqrt{-1} \tau_{3}\right) / 2\right) \in \operatorname{Im} \iota_{\mathbb{C}}$. Then, by some $h \in$ Lie $\mathcal{G}_{00}^{\mathbb{C}}$, we can write

$$
\begin{aligned}
& \frac{1}{2}\left(\tau_{0}+\sqrt{-1} \tau_{1}\right)=[h, \alpha]-\frac{1}{2} \frac{d}{d s} h, \\
& \frac{1}{2}\left(\tau_{2}+\sqrt{-1} \tau_{3}\right)=[h, \beta] .
\end{aligned}
$$

Since $\left(\tau_{0}, \tau_{1}, \tau_{2}, \tau_{3}\right) \in T \mathcal{M}_{\mathrm{hK}}$, they satisfy $\frac{d}{d s}\left(\tau_{0}+\sqrt{-1} \tau_{1}\right)+2\left[\tau_{0}+\sqrt{-1} \tau_{1}, \alpha^{*}\right]+2\left[\tau_{2}+\right.$ 
$\left.\sqrt{-1} \tau_{3}, \beta^{*}\right]=0$. Then we have

$$
\begin{aligned}
0 & =\int_{0}^{1} \operatorname{tr}\left\{\frac{d}{d s}\left([h, \alpha]-\frac{1}{2} \frac{d}{d s} h\right)+2\left[[h, \alpha]-\frac{1}{2} \frac{d}{d s} h, \alpha^{*}\right]+2\left[[h, \beta], \beta^{*}\right]\right\} h^{*} d s \\
& =\int_{0}^{1} \operatorname{tr}\left\{-\left([h, \alpha]-\frac{1}{2} \frac{d}{d s} h\right) \frac{d}{d s} h^{*}-\left[\frac{d}{d s} h, \alpha^{*}\right] h^{*}+2\left[[h, \alpha], \alpha^{*}\right] h^{*}+2\left[\left[h, \beta^{*}\right], \beta\right] h^{*}\right\} d s \\
& =\int_{0}^{1} \operatorname{tr}\left\{2\left(-\frac{1}{2} \frac{d}{d s} h+[h, \alpha]\right)\left(-\frac{1}{2} \frac{d}{d s} h^{*}+[h, \alpha]^{*}\right)+2[h, \beta][h, \beta]^{*}\right\} d s \\
& =2\left\|\left([h, \alpha]-\frac{1}{2} \frac{d}{d s} h,[h, \beta]\right)\right\|_{L^{2}}^{2},
\end{aligned}
$$

where we used integration by parts in the second equality. This implies the injectivity of $F_{*}$. The surjectivity of $F_{*}$ follows from the injectivity.

Next, the holomorphy of $F$ and $G$ follows from the commutativity of the diagram

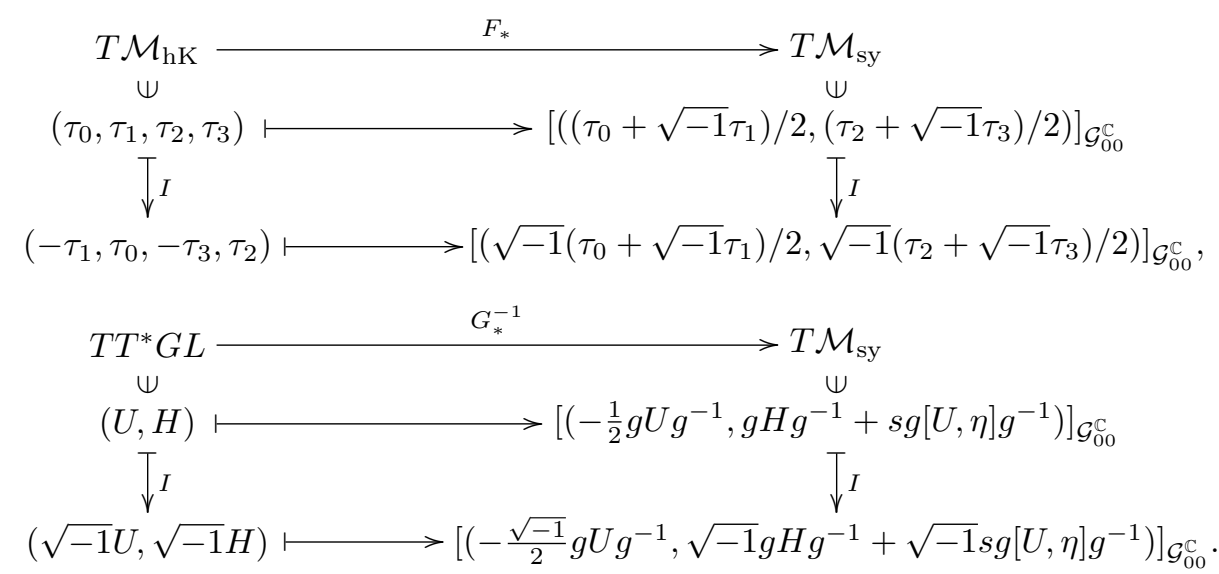

Hence we have the following proposition.

Proposition 3.9. The diffeomorphisms Fand $G$ are holomorphic.

Last, we consider the correspondence of their symplectic forms. The following argument also appears in $[\mathbf{B i}]$.

Lemma 3.10. The holomorphic maps $F$ and $G$ preserve the symplectic forms.

Proof. The assertion for $F$ is clear. Let us check the symplectic form of $\mathcal{M}_{\text {sy }}$ is sent to that of $T^{*} G L$ by $G^{-1^{*}}$. We have

$$
\begin{aligned}
G^{-1^{*}} & \omega\left((U, H),\left(U^{\prime}, H^{\prime}\right)\right) \\
& =\omega\left(\left(-\frac{1}{2} g U g^{-1}, g H g^{-1}+s g[U, \eta] g^{-1}\right),\left(-\frac{1}{2} g U^{\prime} g^{-1}, g H^{\prime} g^{-1}+s g\left[U^{\prime}, \eta\right] g^{-1}\right)\right) \\
& =2 \int_{0}^{1} \operatorname{tr}\left\{-g U g^{-1}\left(g H^{\prime} g^{-1}+s g\left[U^{\prime}, \eta\right] g^{-1}\right)+g U^{\prime} g^{-1}\left(g H g^{-1}+s g[U, \eta] g^{-1}\right)\right\} d s \\
& =-2 \operatorname{tr}\left\{\left(U H^{\prime}-U^{\prime} H\right)+\left(\left[U, U^{\prime}\right]\right) \eta\right\},
\end{aligned}
$$

this coincides with (2.12).

Therefore, the proof of Kronheimer's theorem is completed. 
Corollary 3.12. Take $l \in \mathbb{R}_{>0}$ and suppose the symplectic form of $T^{*} G L$ is given by $l \Omega$ instead of $\Omega$. Then $T^{*} G L$ is symplectomorphic to the moduli space of the solutions of Nahm's equations on the interval $[0, l]$.

Proof. On $[0, l]$ instead of $\mathcal{I}=[0,1]$, the integrand at (3.11) is replaced by

$$
\operatorname{tr}\left\{-g U H^{\prime} g^{-1}+g U^{\prime} H g^{-1}+\frac{s}{l} g\left(-U\left[U^{\prime}, \eta\right]+[U, \eta] U^{\prime}\right) g^{-1}\right\} .
$$

Integrating from 0 to $l$, we have

$$
\begin{aligned}
G^{-1^{*}} \omega\left((U, H),\left(U^{\prime}, H^{\prime}\right)\right) & =-2 l \operatorname{tr}\left\{\left(U H^{\prime}-U^{\prime} H\right)+\left(\left[U, U^{\prime}\right]\right) \eta\right\} \\
& =l \Omega\left((U, H),\left(U^{\prime}, H^{\prime}\right)\right) .
\end{aligned}
$$

Proposition 3.13. In the case of $n=1$ and on $[0, l]$, the biholomorphism $\mathcal{M}_{h K} \cong$ $T^{*} \mathbb{C}^{*} \cong S^{1} \times \mathbb{R}^{3}$ is explicitly given by

$$
\left[\left(T_{0}, T_{1}, T_{2}, T_{3}\right)\right]_{\mathcal{G}_{00}} \mapsto\left(\exp \left(\int_{0}^{l} T_{0} d s\right),-\sqrt{-1} T_{1}(0),-\sqrt{-1} T_{2}(0),-\sqrt{-1} T_{3}(0)\right) \in S^{1} \times \mathbb{R}^{3},
$$

and the symplectic form is given by $\omega=-l\left(d \int_{0}^{l} T_{0} d s+\sqrt{-1} d T_{1}\right) \wedge\left(-d T_{2}+\sqrt{-1} d T_{3}\right)$.

Proof. In this case, Nahm's equations are written by $d T_{k} / d s=0(k=1,2,3)$, so $\left\{\left(T_{1}, T_{2}, T_{3}\right)\right\} \cong(\sqrt{-1} \mathbb{R})^{3}$. By the $\mathcal{G}_{00}$-action, we can regard $T_{0}$ is constant. Put $t_{k}=$ $-\sqrt{-1} T_{k} \in \mathbb{R}$ for $k=0,1,2,3$. $\exp (2 \pi \sqrt{-1} s / l) \in \mathcal{G}_{00}$ sends $t_{0}$ to $t_{0}-2 \pi l^{-1}$ so $\left[t_{0}\right]$ defines an element of $\mathbb{R} / 2 \pi l^{-1} \mathbb{Z}$. Note that we have $\left[\sqrt{-1} t_{0}\right]=\left[\int_{0}^{l} T_{0} d s\right]$. Let $g$ be a solution of $g \cdot 0=\sqrt{-1} t_{0}-t_{1}, g(0)=$ id. Then we have $g(s)=\exp \left\{-\left(\sqrt{-1} t_{0}-t_{1}\right) s\right\}$, $u=g(l)=\exp \left\{-l\left(\sqrt{-1} t_{0}-t_{1}\right)\right\}$ and $\eta=\left(\sqrt{-1} t_{2}-t_{3}\right) / 2$. The symplectic form is given by

$$
\omega=2 u^{-1} d u \wedge d \eta=-l\left(\sqrt{-1} d t_{0}-d t_{1}\right) \wedge\left(\sqrt{-1} d t_{2}-d t_{3}\right) .
$$

\section{Elemental bow varieties}

In this section, we introduce elemental bow varieties and research them in the same way as $[\mathbf{N a 2}]$. The definition of elemental bow varieties is based on Cherkis' work ([C1, $\S 2],[\mathbf{C 2}, \S 2])$. By using elemental bow varieties we will construct ALF spaces in the following section.

In this paper, we define ALF (i.e. Asymptotically Locally Flat) spaces as follows:

Definition 4.1 (c.f. $[\mathbf{M}]$ ). A connected complete hyper-Kähler 4-manifold $(M, g)$ is called an ALF space if there exist $c_{1}$ and $c_{2}\left(0<c_{1} \leq c_{2}\right)$ such that for any $x \in M$ and for any $r \geq 1$, the metric $g$ satisfies

$$
c_{1} r^{3} \leq \operatorname{Vol}_{g} B(x, r) \leq c_{2} r^{3} .
$$

For example, $\mathbb{R}^{3} \times S^{1}$ is an ALF space. For nontrivial example, Taub-NUT space is ALF. Taub-NUT space is homeomorphic to $\mathbb{C}^{2}$ and has a hyper-Kähler structure, and its metric is given by

$$
g_{\mathrm{TN}}=\left(\lambda+\frac{1}{|x|}\right) d x^{2}+\left(\lambda+\frac{1}{|x|}\right)^{-1}(d \psi+\omega)^{2},
$$


where $x \in \mathbb{R}^{3}, \psi \in S^{1}, d \omega=* d \frac{1}{|x|}$ and $\lambda>0$ is a parameter. As $|x| \rightarrow \infty$, we have $g_{\mathrm{TN}} \rightarrow d x^{2}+(d \psi+\omega)^{2}$, so the metric $g_{\mathrm{TN}}$ actually satisfies the condition about the volume growth.

\subsection{Elemental bows}

For $l>0$, we consider the following diagram:

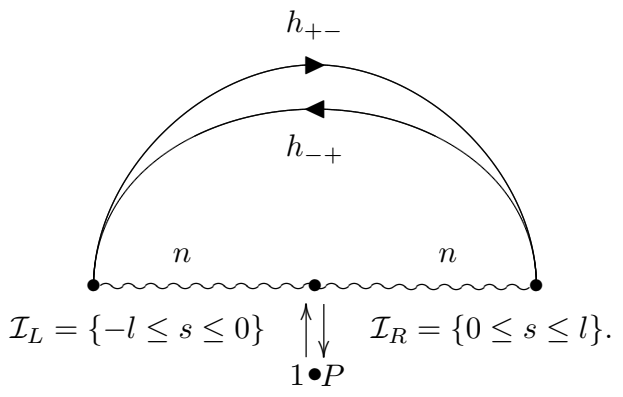

We call this diagram (with the following data) an elemental bow. We give a rank- $n$ hermitian vector space $W \cong \mathbb{C}$ on $P$ and a trivial hermitian vector bundle $\pi: V \rightarrow$ $\mathcal{I}_{L} \cup \mathcal{I}_{R}$, and describe $\pi^{-1}(s)$ as $V(s)$. Let us define complex vector spaces $\mathbb{M}_{\Omega}, \mathbb{M}_{\bar{\Omega}}$ and $\mathbb{M}$ respectively by

$$
\begin{aligned}
\mathbb{M}_{\Omega} & =\operatorname{Hom}(V(l), V(-l)) \oplus \operatorname{Hom}(W, V(0))=\left\{\left(B_{-+}, i\right) \in M(n, n ; \mathbb{C}) \oplus M(1, n ; \mathbb{C})\right\}, \\
\mathbb{M}_{\bar{\Omega}} & =\operatorname{Hom}(V(-l), V(l)) \oplus \operatorname{Hom}(V(0), W)=\left\{\left(B_{+-}, j\right) \in M(n, n ; \mathbb{C}) \oplus M(n, 1 ; \mathbb{C})\right\}, \\
\mathbb{M} & =\mathbb{M}_{\Omega} \oplus \mathbb{M}_{\bar{\Omega}} \oplus \mathcal{H}_{L} \oplus \mathcal{H}_{R},
\end{aligned}
$$

where $\mathcal{H}_{\bullet} \mathrm{s}$ are as in $\S 2.1$. The metric of $\mathbb{M}$ is given by

$$
\begin{aligned}
d s^{2}=\operatorname{tr}\left\{d B_{-+} d B_{-+}^{*}+\right. & \left.d B_{+-} d B_{+-}^{*}+d i d i^{*}+d j^{*} d j\right\} \\
& +\sum_{A=L, R} \int_{\mathcal{I}_{A}} \operatorname{tr}\left\{d T_{0}^{A} d\left(T_{0}^{A}\right)^{*}+d T_{k}^{A} d\left(T_{k}^{A}\right)^{*}\right\} d s,
\end{aligned}
$$

where the meaning of the integrand is as (2.1) and $X^{*}$ means Hermitian adjoint ${ }^{t} \bar{X}$. We define a complex structure $J$ as $J\left(B_{-+}, B_{+-}, i, j\right):=\left(-B_{+-}^{*}, B_{-+}^{*},-j^{*}, i^{*}\right)$, then $\mathbb{M}$ has a hyper-Kähler structure. The Kähler forms are written as

$$
\begin{aligned}
\omega_{I}= & \frac{\sqrt{-1}}{2} \operatorname{tr}\left\{d B_{-+} \wedge d B_{-+}^{*}+d B_{+-} \wedge d B_{+-}^{*}+d i \wedge d i^{*}+d j \wedge d j^{*}\right\} \\
& +\sum_{A=L, R} \int_{\mathcal{I}_{A}}-\operatorname{tr}\left\{d T_{0}^{A} \wedge d T_{1}^{A}+d T_{2}^{A} \wedge d T_{3}^{A}\right\} d s, \\
\omega_{J}+\sqrt{-1} \omega_{K}= & \operatorname{tr}\left\{d B_{-+} \wedge d B_{+-}+d i \wedge d j\right\} \\
& +\sum_{A=L, R} \int_{\mathcal{I}_{A}} \operatorname{tr}\left\{\left(d T_{0}^{A}+\sqrt{-1} d T_{1}^{A}\right) \wedge\left(d T_{2}^{A}+\sqrt{-1} d T_{3}^{A}\right)\right\} d s .
\end{aligned}
$$

A group $\mathcal{G}=\left\{g=\left(g_{L}, g_{R}\right) \in L_{2}^{2}\left(\mathcal{I}_{L}, U(n)\right) \times L_{2}^{2}\left(\mathcal{I}_{R}, U(n)\right) \mid g_{L}(0)=g_{R}(0)\right\}$ acts on $\mathbb{M}$ by

$$
\begin{aligned}
g \cdot( & \left.B_{-+}, B_{+-}, i, j, T_{0}^{A}(s), T_{k}^{A}(s)\right) \\
\quad & =\left(g(-l) B_{-+} g(l)^{-1}, g(l) B_{+-} g(-l)^{-1}, g(0) i, j g(0)^{-1}, g T_{0}^{A} g^{-1}-\frac{d g}{d s} g^{-1}, g T_{k}^{A} g^{-1}\right),
\end{aligned}
$$


for $k=1,2,3, A=L, R$. This action preserves the hyper-Kähler structure. Then we have a hyper-Kähler moment map $\mu$ (see also Remark 2.5 and $[\mathbf{K r} \mathbf{2}]$ ). The explicit form is as follows:

$$
\begin{aligned}
\mu_{\mathbb{R}}= \begin{cases}\frac{-\sqrt{-1}}{2}\left(B_{-+} B_{-+}^{*}-B_{+-}^{*} B_{+-}\right)+T_{1}^{L}(-l) & \text { at } s=-l \\
\frac{d}{d s} T_{1}^{A}+\left[T_{0}^{A}, T_{1}^{A}\right]+\left[T_{2}^{A}, T_{3}^{A}\right] & \text { at } s \in(-l, 0),(0,-l) \\
\frac{-\sqrt{-1}}{2}\left(i i^{*}-j^{*} j\right)-T_{1}^{L}(0)+T_{1}^{R}(0) & \text { at } s=0 \\
\frac{-\sqrt{-1}}{2}\left(B_{+-} B_{+-}^{*}-B_{-+}^{*} B_{-+}\right)-T_{1}^{R}(l) & \text { at } s=l,\end{cases} \\
\mu_{\mathbb{C}}= \begin{cases}B_{-+} B_{+-}+\left(T_{2}^{l}+\sqrt{-1} T_{3}^{l}\right)(-l) & \text { at } s=-l \\
\frac{d}{d s}\left(T_{2}^{A}+\sqrt{-1} T_{3}^{A}\right)+\left[T_{0}^{A}+\sqrt{-1} T_{1}^{A}, T_{2}^{A}+\sqrt{-1} T_{3}^{A}\right] & \text { at } s \in(-l, 0),(0,-l) \\
i j-\left(T_{2}^{L}+\sqrt{-1} T_{3}^{L}\right)(0)+\left(T_{2}^{R}+\sqrt{-1} T_{3}^{R}\right)(0) & \text { at } s=0 \\
-B_{+-} B_{-+}-\left(T_{2}^{R}+\sqrt{-1} T_{3}^{R}\right)(l) & \text { at } s=l .\end{cases}
\end{aligned}
$$

Put $Z=\left\{\zeta \in \operatorname{Lie}(U(n))^{*} \otimes(\mathbb{R} \oplus \mathbb{C}) \mid \operatorname{Ad}_{g(0)}^{*}(\zeta)=\zeta\right.$ for all $\left.g \in \mathcal{G}\right\}$. Choose an element $\zeta=\left(\zeta_{\mathbb{R}}, \zeta_{\mathbb{C}}\right) \in Z$, and define a hyper-Kähler quotient $\mathcal{M}_{\zeta}(n, l)$ of $\mathbb{M}$ by $\mathcal{G}$ as follows:

$$
\mathcal{M}_{\zeta}(n, l):=\left\{(B, i, j, T) \in \mathbb{M} \mid \mu(B, i, j, T)=\zeta \delta_{0}\right\} / \mathcal{G} .
$$

Here $\delta_{0}$ is Dirac's delta on $\mathcal{I}_{L} \cup \mathcal{I}_{R}$ i.e. $\delta_{0}(0)=1$ and $\delta_{0}(s)=0$ for $s \neq 0$. We call this hyper-Kähler manifold $\mathcal{M}_{\zeta}(n, l)$ an elemental bow variety.

REMARK 4.5. (i) As a usual hyper-Kähler quotient, we may have to change $Z$ into $Z^{\prime}=\left\{\zeta^{\prime} \in\right.$ Lie $\mathcal{G}^{*} \otimes(\mathbb{R} \oplus \mathbb{C}) \mid \operatorname{Ad}_{g}^{*}\left(\zeta^{\prime}\right)=\zeta^{\prime}$ for all $\left.g \in \mathcal{G}\right\}$. However, by a change of coordinates, we get $\mathcal{M}_{\zeta^{\prime}} \cong \mathcal{M}_{\zeta}$ for a certain $\zeta \in Z$. Thus we do not need $Z^{\prime}$ and at insides of intervals $\mu(B, i, j, T)=\zeta \delta_{0}$ means $\{T\}$ satisfies Nahm's equations (see also Remark 2.5).

(ii) $\zeta$ can be written as $\left(\zeta_{\mathbb{R}}, \zeta_{\mathbb{C}}\right)=\left(c_{\mathbb{R}} \sqrt{-1} \mathrm{id}, c_{\mathbb{C}}\right.$ id $)$, where $c_{\mathbb{R}}=\operatorname{tr} \zeta_{\mathbb{R}} / n \sqrt{-1} \in \mathbb{R}, c_{\mathbb{C}}=$ $\operatorname{tr} \zeta_{\mathbb{C}} / n \in \mathbb{C}$.

We apply Kronheimer's theorem to $\mathcal{M}_{\zeta}(n, l)$, we have

$$
\mathcal{M}_{\zeta}(n, l) \cong\left\{(B, i, j, u, \eta) \in \mathbb{M}_{\mathbb{C}} \mid \mu(B, i, j, u, \eta)=\zeta \delta_{0}\right\} / G,
$$

where we put $\mathbb{M}_{\mathbb{C}}=\mathbb{M}_{\Omega} \oplus \mathbb{M}_{\bar{\Omega}} \oplus T^{*} G L(n) \oplus T^{*} G L(n)$ and $G=U(n) \times U(n) \times U(n)$. We describe these data as the following diagram:

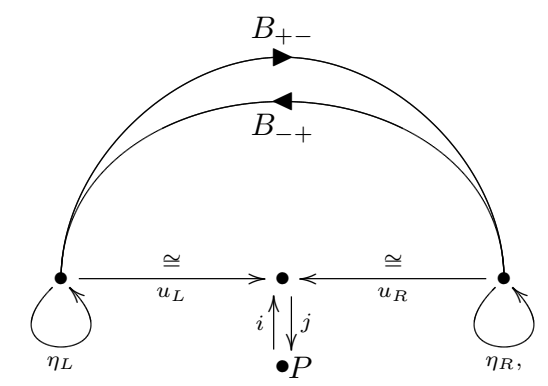

where $\cong$ means Ker $u_{A}=0$. The real moment map cannot be described explicitly by 
using $u$ and $\eta$, but the complex moment map can be described as

$$
\mu_{\mathbb{C}}(B, i, j, u, \eta)= \begin{cases}B_{-+} B_{+-}+2 \eta_{L} & \text { at } s=-l \\ i j-2 u_{L} \eta_{L} u_{L}^{-1}+2 u_{R} \eta_{R} u_{R}^{-1} & \text { at } s=0 \\ -B_{+-} B_{-+}-2 \eta_{R} & \text { at } s=l .\end{cases}
$$

And the group $G$ acts on $\{(u, \eta)\}$ as

$$
\left(g_{-}, g_{0}, g_{+}\right):\left(u_{L}, \eta_{L}, u_{R}, \eta_{R}\right) \mapsto\left(g_{0} u_{L} g_{-}^{-1}, g_{-} \eta_{L} g_{-}^{-1}, g_{0} u_{R} g_{+}^{-1}, g_{+} \eta_{R} g_{+}^{-1}\right) .
$$

\subsection{General properties of elemental bow varieties}

In the last subsection, we claimed that elemental bow varieties can be constructed as finite dimensional hyper-Kähler quotient. By considering the relation between finite dimensional hyper-Kähler quotient and GIT quotient, we prove the following proposition.

Proposition 4.7. The elemental bow variety $\mathcal{M}_{\zeta}(n, l)$ is algebraic.

Proof. We prove the hyper-Kähler quotient $\mu_{\mathbb{R}}^{-1}\left(\zeta_{\mathbb{R}}\right) \cap \mu_{\mathbb{C}}^{-1}\left(\zeta_{\mathbb{C}}\right) / G$ is biholomorphic to the GIT quotient $\mu_{\mathbb{C}}^{-1}\left(\zeta_{\mathbb{C}}\right) /{ }_{\chi} G_{\mathbb{C}}$. By the same argument as [Kr2, Corollary 3.12], it is enough to consider in the case of $\zeta_{\mathbb{C}}=0$. According to the argument in $\S 1 \cdot 2 \cdot 2$, first we consider a prequantisation of $\mu_{\mathbb{C}}^{-1}(0)$.

We take a trivial line bundle $L$ on $\mu_{\mathbb{C}}^{-1}(0)$ and give a hermitian structure by a function $h\left(z_{p}\right)=|z|^{2} e^{f(p)}$, where $z_{p}=(p, z) \in \mu_{\mathbb{C}}^{-1}(0) \times \mathbb{C} \cong L$. Here we give $f(B, i, j, u, \eta)=$ $f_{1}(u, \eta)+f_{2}(B)+f_{3}(i, j)$, and

$$
\begin{aligned}
f_{1}(u, \eta) & =\frac{1}{2} \sum_{A=L, R} \int_{\mathcal{I}_{A}}\left\|\alpha_{A}(s)+\alpha_{A}^{*}(s)\right\|^{2}+2\left\|\beta_{A}(s)\right\|^{2} d s, \\
f_{2}\left(B_{-+}, B_{+-}\right) & =\frac{1}{4}\left(\left\|B_{-+}\right\|^{2}+\left\|B_{+-}\right\|^{2}\right), \\
f_{3}(i, j) & =\frac{1}{4}\left(\|i\|^{2}+\|j\|^{2}\right),
\end{aligned}
$$

where $(\alpha, \beta)$ satisfies Nahm's equation and $G(\alpha, \beta)=(u, \eta) \in T^{*} G L$ (c.f. $\left.\S 2.4\right)$. In fact this line bundle $(L, h)$ is a prequantisation of $\mu_{\mathbb{C}}^{-1}(0)$. It is because we have

$$
\begin{aligned}
(\partial-\bar{\partial}) f_{1}(u, \eta) & =\int \operatorname{tr}\left\{\left(\alpha+\alpha^{*}\right)\left(d \alpha-d \alpha^{*}\right)+d \beta \beta^{*}-\beta d \beta^{*}\right\} d s, \\
d(\partial-\bar{\partial}) f_{1}(u, \eta) & =\int \operatorname{tr}\left\{\left(d \alpha+d \alpha^{*}\right) \wedge\left(d \alpha-d \alpha^{*}\right)-d \beta \wedge d \beta^{*}-d \beta \wedge d \beta^{*}\right\} d s \\
& =-\int 2 \operatorname{tr}\left\{d \alpha \wedge d \alpha^{*}+d \beta \wedge d \beta^{*}\right\} d s \\
& =-\sqrt{-1} \int \operatorname{tr}\left\{d T_{0} \wedge d T_{1}+d T_{2} \wedge d T_{3}\right\} d s, \\
(\partial-\bar{\partial}) f_{2}\left(B_{-+}, B_{+-}\right) & =\frac{1}{4} \operatorname{tr}\left\{\left(d B_{-+} B_{-+}^{*}-B_{-+} d B_{-+}^{*}\right)+\left(d B_{+-} B_{+-}^{*}-B_{+-} d B_{+-}^{*}\right)\right\}, \\
d(\partial-\bar{\partial}) f_{2}\left(B_{-+}, B_{+-}\right) & =-\frac{1}{2} \operatorname{tr}\left\{d B_{-+} \wedge d B_{-+}^{*}+d B_{+-} \wedge d B_{+-}^{*}\right\}, \\
d(\partial-\bar{\partial}) f_{3}(i, j) & =-\frac{1}{2} \operatorname{tr}\left\{d i \wedge d i^{*}+d j \wedge d j^{*}\right\},
\end{aligned}
$$

and the summation of the fourth, sixth and seventh of right hand sides coincide with $\sqrt{-1} \omega_{I}$. 
On the other hand, since $L$ is trivial, a linearisation of the $G_{\mathbb{C}}$-action can be described as $g \cdot(p, z)=\left(g \cdot p, \chi(g)^{-1} z\right)$, where $\chi$ is a complexification of a character $\chi^{\mathbb{R}}: G \rightarrow U(1)$. Suppose

$$
\chi(g)^{-1}=\left(\operatorname{det} g_{-}\right)^{c_{-}}\left(\operatorname{det} g_{0}\right)^{c_{0}}\left(\operatorname{det} g_{+}\right)^{c_{+}}, \quad c_{\bullet} \in \mathbb{R} .
$$

By Proposition 1.10, this linearisation determines the moment map $\mu_{\chi}$.

Recall that $\left\langle\mu_{\chi}(B, i, j, u, \eta), \xi\right\rangle$ is given by $s^{*} \iota_{\tilde{\xi}} A$, where $s: M \rightarrow L$ is the 0 -section. Here the connection form $A$ of $L$ (see (1.8)) is given by

$$
\begin{aligned}
A=\frac{1}{4 \sqrt{-1}}\left(4 \frac{d z}{z}-4 \frac{d \bar{z}}{\bar{z}}\right. & +\sum_{A=L, R} \int_{\mathcal{I}_{A}} 4 \operatorname{tr}\left\{\left(\alpha_{A}+\alpha_{A}^{*}\right)\left(d \alpha_{A}-d \alpha_{A}^{*}\right)+d \beta_{A} \beta_{A}^{*}-\beta_{A} d \beta_{A}^{*}\right\} d s \\
& +\operatorname{tr}\left\{\left(d B_{-+} B_{-+}^{*}-B_{-+} d B_{-+}^{*}\right)+\left(d B_{+-} B_{+-}^{*}-B_{+-} d B_{+-}^{*}\right)\right\} \\
& \left.+\operatorname{tr}\left\{\left(d i i^{*}-i d i^{*}\right)+\left(d j j^{*}-j d j^{*}\right)\right\}\right) .
\end{aligned}
$$

We identify $\exp (\xi) \in G$ with $\exp (\xi(s)) \in \mathcal{G} / \mathcal{G}_{000}$, where $\mathcal{G}_{000}=\{g \in \mathcal{G} \mid g(-l)=g(0)=$ $g(l)=\mathrm{id}\}$. Since $\xi^{*}=-\xi$, we have

$$
\begin{aligned}
-\iota_{\tilde{\xi}} d z & =\left(c_{-} \operatorname{tr} \xi(-l)+c_{0} \operatorname{tr} \xi(0)+c_{+} \operatorname{tr} \xi(l)\right) z, \\
-\iota_{\tilde{\xi}} d \bar{z} & =-\left(c_{-} \operatorname{tr} \xi(-l)+c_{0} \operatorname{tr} \xi(0)+c_{+} \operatorname{tr} \xi(l)\right) \bar{z}, \\
-\iota_{\tilde{\xi}} d \alpha & =[\xi(s), \alpha(s)]-\frac{1}{2} \frac{d}{d s} \xi(s), \\
-\iota_{\tilde{\xi}} d \beta & =[\xi(s), \beta(s)], \\
-\iota_{\tilde{\xi}} d B_{-+} & =\xi(-l) B_{-+}-B_{-+} \xi(l), \\
-\iota_{\tilde{\xi}} d i & =\xi(0) i,
\end{aligned}
$$

Hence we get

$$
\begin{aligned}
\left\langle 4 \sqrt{-1} \mu_{\chi}, \xi\right\rangle= & 8\left(c_{-} \operatorname{tr} \xi(-l)+c_{0} \operatorname{tr} \xi(0)+c_{+} \operatorname{tr} \xi(l)\right) \\
& +\sum_{A=L, R} \int_{\mathcal{I}_{A}} 4 \operatorname{tr}\left\{\left(\alpha_{A}+\alpha_{A}^{*}\right)\left(\left[\xi, \alpha_{A}\right]-\frac{1}{2} \frac{d}{d s} \xi+\left[\alpha_{A}^{*}, \xi\right]-\frac{1}{2} \frac{d}{d s} \xi\right)\right. \\
& \left.+\left[\xi, \beta_{A}\right] \beta_{A}^{*}+\beta_{A}\left[\beta_{A}^{*}, \xi\right]\right\} d s \\
+ & \operatorname{tr}\left\{\left(\xi(-l) B_{-+}-B_{-+} \xi(l)\right) B_{-+}^{*}-B_{-+}\left(\xi(l) B_{-+}^{*}-B_{-+}^{*} \xi(-l)\right)\right. \\
& \left.\quad+\left(\xi(l) B_{+-}-B_{+-} \xi(-l)\right) B_{+-}^{*}-B_{+-}\left(\xi(-l) B_{+-}^{*}+B_{+-}^{*} \xi(l)\right)\right\} \\
& +\operatorname{tr}\left\{\xi(0) i i^{*}-i\left(-i^{*} \xi(0)\right)+(-j \xi(0)) j^{*}-j \xi(0) j^{*}\right\} \\
= & \operatorname{tr}\left\{8 c_{+}-4\left(\alpha_{R}(l)+\alpha_{R}^{*}(l)\right)+2\left(B_{+-} B_{+-}^{*}-B_{-+}^{*} B_{-+}\right)\right\} \xi(l) \\
& +\operatorname{tr}\left\{8 c_{0}+4\left(\alpha_{R}(0)+\alpha_{R}^{*}(0)\right)-4\left(\alpha_{L}(0)+\alpha_{L}^{*}(0)\right)+2\left(i i^{*}-j^{*} j\right)\right\} \xi(0) \\
& +\operatorname{tr}\left\{8 c_{-}+4\left(\alpha_{L}(-l)+\alpha_{L}^{*}(-l)\right)+2\left(B_{-+} B_{-+}^{*}-B_{+-}^{*} B_{+-}\right)\right\} \xi(-l) .
\end{aligned}
$$

Here we used integration by parts and the assumption that $(\alpha, \beta)$ is the solution of Nahm's equations. This implies

$$
\mu_{\chi}(B, i, j, u, \eta)=\left\{\begin{array}{lc}
\mu_{\mathbb{R}}-2 c_{-} \sqrt{-1} \text { id } \quad \text { at } s=-l \\
\mu_{\mathbb{R}}-2 c_{0} \sqrt{-1} \text { id } \quad \text { at } s=0 \\
\mu_{\mathbb{R}}-2 c_{+} \sqrt{-1} \text { id } \quad \text { at } s=l .
\end{array}\right.
$$


Bow varieties and $A L F$ spaces

Instead of (4.8), we define a linearisation by putting

$$
\chi(g)^{-1}=\left(\operatorname{det} g_{0}\right)^{\frac{\operatorname{tr} \zeta_{\mathbb{R}}}{2 n \sqrt{-1}}} .
$$

Then we apply Lemma 1.11 to this linearisation, we have a biholomorphism

$$
\left\{\mu_{\mathbb{C}}^{-1}(0)\right\}^{\text {ass }} / G_{\mathbb{C}} \cong \mu_{\chi}^{-1}(0) \cap \mu_{\mathbb{C}}^{-1}(0) / G=\mu_{\mathbb{R}}^{-1}\left(\zeta_{\mathbb{R}}\right) \cap \mu_{\mathbb{C}}^{-1}(0) / G=\mathcal{M}_{\zeta}(n, l) .
$$

On the other hand, we can consider the GIT quotient $\mu_{\mathbb{C}}^{-1}(0) / /{ }_{\chi} G_{\mathbb{C}}$, because the above linearisation is trivial. We claim that $\mu_{\mathbb{C}}^{-1}(0)^{\text {ass }}$ coincides with $\mu_{\mathbb{C}}^{-1}(0)^{s s}$. We can make the similar argument as [Na2, Proposition 3.9., 3.21.] to prove this claim. The difference is that $\|x\|$ is replaced by $f$. In [Na2, p.32, 35], he used the properness of $x \mapsto\|x\|$ so it is enough to check the properness of $f$. For $f_{2}$ and $f_{3}$ it is clear, and for $f_{1}$ the compactness of the subset $\left\{(u, \eta) \mid f_{1}(u, \eta) \leq C\right\}$ follows from the argument of Lemma 3.3.

Therefore we have

$$
\mathcal{M}_{\zeta}(n, l)=\mu_{\mathbb{R}}^{-1}\left(\zeta_{\mathbb{R}}\right) \cap \mu_{\mathbb{C}}^{-1}(0) / G \cong \mu_{\mathbb{C}}^{-1}(0) / \chi_{\chi} G_{\mathbb{C}},
$$

and the elemental bow variety $\mathcal{M}_{\zeta}(n, l)$ is actually an algebraic variety because $\mu_{\mathbb{C}}^{-1}(0)$ $\| / G_{\mathbb{C}}$ is algebraic. (When $\zeta_{\mathbb{R}}=0, \mu_{\mathbb{C}}^{-1}(0) / / G_{\mathbb{C}}$ is regarded as an affine algebrogeometric quotient because $\chi=\mathrm{id}$.)

We give another characterisation to the (semi)stability condition of the GIT quotient $\mu_{\mathbb{C}}^{-1}(0) / / \chi G_{\mathbb{C}}$. Here we recall that $(B, i, j, u, \eta)$ is called a $(\chi-)$ semistable point when $G_{\mathbb{C}} \cdot(B, i, j, u, \eta, z)$ is closed for $z \neq 0$, and $(B, i, j, u, \eta)$ is called a stable point when $G_{\mathbb{C}} \cdot(B, i, j, u, \eta)$ is closed and its stabiliser is finite (see also $\left.\$ 1.2\right)$.

Definition 4.9. For $V=\{V(-l), V(0), V(l)\}$, a set of three subspaces $S=\{S(-l)$, $S(0), S(l)\},(S(s) \subset V(s))$ is called invariant for $(B, i, j, u, \eta)$ if it satisfies the following conditions:

$$
\begin{aligned}
& B_{-+}(S(l)) \subset S(-l), \quad u_{L}(S(-l))=S(0), \quad \eta_{L}(S(-l)) \subset S(-l), \\
& B_{+-}(S(-l)) \subset S(l), \quad u_{R}(S(l))=S(0), \quad \eta_{R}(S(l)) \subset S(l) .
\end{aligned}
$$

Theorem 4.10. Put $x=(B, i, j, u, \eta) \in \mu_{\mathbb{C}}^{-1}(0)$ and $c=\frac{\operatorname{tr} \zeta_{\mathbb{R}}}{2 n \sqrt{-1}} \in \mathbb{R}$.

(i) When $c<0,(x, z)$ is semistable if and only if there exists no set of proper subspaces $S \subsetneq V$ such that $S$ is invariant for $x$ and $\operatorname{Im} i \subset S(0)$.

(ii) When $c>0,(x, z)$ is semistable if and only if there exists no set of nonzero subspaces $0 \neq S \subset V$ such that $S$ is invariant for $x$ and $S(0) \subset \operatorname{Ker} j$.

(iii) When $c=0, x$ is stable if and only if there exists no set of nonzero proper subspaces $0 \neq S \subsetneq V$ such that $S$ is invariant for $x$ and $\operatorname{Im} i \subset S(0) \subset \operatorname{Ker} j$.

Proof. The proofs of three assertions are analogous. First we suppose the orbit $G_{\mathbb{C}}$. $(x, z)$ is closed for $z \neq 0$ and there exists an invariant set of subspaces $S \subset V$. If $\operatorname{Im} i \subset S(0)$, we take

$$
\begin{aligned}
g_{-}^{t} & =\left(\mathrm{id}, t^{-1} \mathrm{id}\right) \in G L(S(-l)) \times G L\left(S(-l)^{\perp}\right) \subset G L(V(-l)), \\
g_{0}^{t} & =\left(\mathrm{id}, t^{-1} \mathrm{id}\right) \in G L(S(0)) \times G L\left(S(0)^{\perp}\right) \subset G L(V(0)), \\
g_{+}^{t} & =\left(\mathrm{id}, t^{-1} \mathrm{id}\right) \in G L(S(l)) \times G L\left(S(l)^{\perp}\right) \subset G L(V(l)) .
\end{aligned}
$$


Then we have $g_{0}^{t} u_{L}\left(g_{-}^{t}\right)^{-1}=u_{L}, g_{0}^{t} u_{R}\left(g_{+}^{t}\right)^{-1}=u_{R}$ and

$$
\begin{aligned}
\left.g_{-}^{t} B_{-+}\left(g_{+}^{t}\right)^{-1}\right|_{S(l)^{\perp} \rightarrow S(-l)} & =\left.t B_{-+}\right|_{S(l)^{\perp} \rightarrow S(-l)}, \\
\left.g_{+}^{t} B_{+-}\left(g_{-}^{t}\right)^{-1}\right|_{S(-l)^{\perp} \rightarrow S(l)} & =\left.t B_{+-}\right|_{S(-l)^{\perp} \rightarrow S(l)}, \\
\left.g_{-}^{t} \eta_{L}\left(g_{-}^{t}\right)^{-1}\right|_{S(-l)^{\perp} \rightarrow S(-l)} & =\left.t \eta_{L}\right|_{S(-l)^{\perp} \rightarrow S(-l)}, \\
\left.g_{+}^{t} \eta_{R}\left(g_{+}^{t}\right)^{-1}\right|_{S(l)^{\perp} \rightarrow S(l)} & =\left.t \eta_{R}\right|_{S(l)^{\perp} \rightarrow S(l)}, \\
g_{0}^{t} i=i,\left.\quad j\left(g_{0}^{t}\right)^{-1}\right|_{S(0)^{\perp} \rightarrow W} & =\left.t j\right|_{S(0)^{\perp} \rightarrow W}, \\
\chi\left(g^{t}\right)^{-1} z & =t^{-c \operatorname{dim} S(0)^{\perp} .}
\end{aligned}
$$

And if $\operatorname{Ker} j \supset S(0)$, we take

$$
\begin{aligned}
& g^{\prime t}=(t \text { id, id }) \in G L(S(-l)) \times G L\left(S(-l)^{\perp}\right) \subset G L(V(-l)), \\
& g^{\prime t}=(t \text { id, id }) \in G L(S(0)) \times G L\left(S(0)^{\perp}\right) \subset G L(V(0)), \\
& g^{\prime t}{ }_{+}=(t \text { id, id }) \in G L(S(l)) \times G L\left(S(l)^{\perp}\right) \subset G L(V(l)) .
\end{aligned}
$$

Then we have the same equations for $u_{L}, u_{R}, B_{-+}, B_{+-}, \eta_{L}$ and $\eta_{R}$ as above and

$$
\left.g_{0}^{\prime t} i\right|_{W \rightarrow S(0)}=\left.t i\right|_{W \rightarrow S(0)}, \quad j\left(g_{0}^{\prime t}\right)^{-1}=j, \quad \chi\left(g^{\prime t}\right)^{-1} z=t^{c \operatorname{dim} S(0)} .
$$

Combined with these things, when $c<0$ and $\operatorname{Im} i \subset S(0), S(0)^{\perp} \neq 0$ leads to a contradiction and when $c>0$ and $\operatorname{Ker} j \supset S(0), S(0) \neq 0$ also does.

And when $c=0, \operatorname{Im} i \subset S(0) \subset \operatorname{Ker} j$ implies there exists $x=(B, i, j, u, \eta) \in \mu_{\mathbb{C}}^{-1}(0)$ which satisfies

$$
\left.B_{-+}\right|_{S(l) \perp \rightarrow S(-l)}=\left.B_{+-}\right|_{S(-l)^{\perp} \rightarrow S(l)}=\left.\eta_{L}\right|_{S(-l)^{\perp} \rightarrow S(-l)}=\left.\eta_{R}\right|_{S(l)^{\perp} \rightarrow S(l)}=i=j=0,
$$

by taking the limit $t \rightarrow 0$. The stabiliser of this element is infinite, so this is a contradiction.

Conversely, we suppose that the (semi)stability condition is satisfied and that the orbit is not closed for $z \neq 0$. Then there exists $\lambda=\left(\lambda_{-}, \lambda_{0}, \lambda_{+}\right): \mathbb{C}^{*} \rightarrow G_{\mathbb{C}}$, such that $\lim _{t \rightarrow 0} \lambda(t) \cdot(x, z)$ exists and this limit is contained in $\overline{G_{\mathbb{C}} \cdot(x, z)} \backslash G_{\mathbb{C}} \cdot(x, z)$. Let us take a weight decomposition of $V$ with respect to $\lambda$ :

$$
V(-l)=\oplus_{\alpha} V\left(-l, m_{-, \alpha}\right), V(0)=\oplus_{\alpha} V\left(0, m_{0, \alpha}\right), V(1)=\oplus_{\alpha} V\left(l, m_{+, \alpha}\right),
$$

where $m_{\bullet}, \alpha$ are the weights. Then the existence of $\lim _{t \rightarrow 0} \lambda_{0}(t) u_{L} \lambda_{-}^{-1}(t)$ implies $m_{-, \alpha}=$ $m_{0, \alpha}$ and this also holds for $u_{R}$, we put $m_{\alpha}:=m_{-, \alpha}=m_{0, \alpha}=m_{+, \alpha}$. We can assume $m_{1} \geq m_{2} \geq \cdots \geq m_{n}$. The existence of $\lim _{t \rightarrow 0} \lambda(t) \cdot(x, z)$ implies the following:

$$
\begin{aligned}
B_{-+}\left(V\left(l, m_{\alpha}\right)\right) & \subset \bigoplus_{\beta \geq \alpha} V\left(-l, m_{\beta}\right), & B_{+-}\left(V\left(-l, m_{\alpha}\right)\right) & \subset \bigoplus_{\beta \geq \alpha} V\left(l, m_{\beta}\right), \\
\eta_{L}\left(V\left(-l, m_{\alpha}\right)\right) & \subset \bigoplus_{\beta \geq \alpha} V\left(-l, m_{\beta}\right), & \eta_{R}\left(V\left(l, m_{\alpha}\right)\right) & \subset \bigoplus_{\beta \geq \alpha} V\left(l, m_{\beta}\right), \\
\operatorname{Im} i & \subset \bigoplus_{\beta \geq 0} V\left(0, m_{\beta}\right), & \operatorname{Ker} j & \supset \bigoplus_{\beta \geq 0} V\left(0, m_{\beta}\right) .
\end{aligned}
$$

Thus $\oplus_{\beta \geq \alpha} V\left(m_{\beta}\right)=\left\{V\left(-l, m_{\beta}\right), V\left(0, m_{\beta}\right), V\left(l, m_{\beta}\right)\right\}$ is an invariant set of subspaces.

When $c<0$, we have $V=\bigoplus_{\beta>0} V\left(m_{\beta}\right)$ by the stability condition. Hence $\operatorname{det}(\lambda(t))=$ $t^{N}$ for some $N \geq 0$. If $N=0, V=V(0)$ and $\lambda(t) \equiv 0$ is against $\lim _{t \rightarrow 0} \lambda(t) \cdot(x, z) \notin$ $G_{\mathbb{C}} \cdot(x, z)$. If $N>0, \chi(\lambda(t))^{-1} z=t^{-c N} z$ is against the existence of $\lim \lambda(t) \cdot(x, z)$. 
When $c>0$, we have $V=\bigoplus_{\beta \leq 0} V\left(m_{\beta}\right)$ by the stability condition. This also leads to a contradiction by the same argument.

When $c=0$, we have $V=V(0)$ by the stability condition. This also leads to a contradiction.

Proposition 4.11. As algebraic varieties, the elemental bow variety is isomorphic to the quiver variety given by $\left\{\left[B_{1}, B_{2}\right]+i j=0\right\} / /{ }_{\chi} G L$.

Proof. We give a map and check the map preserves the complex moment map and the stability condition. We define a map

$$
\left(B_{-+}, B_{+-}, u_{A}, \eta_{A}\right) \mapsto\left(B_{1}, B_{2}\right)=\left(u_{L} B_{-+} u_{R}^{-1}, u_{R} B_{+-} u_{L}^{-1}\right) .
$$

Then by (4.6), $\mu_{\mathbb{C}}=0$ corresponds to $\left[B_{1}, B_{2}\right]+i j=0$. We have to consider the induced (semi)stability condition on $\left\{\left[B_{1}, B_{2}\right]+i j=0\right\} / /{ }_{\chi} G L$. By Theorem 4.10, we have the stability condition for $x=\left(B_{1}, B_{2}\right)$ :

(i) When $c<0,(x, z)$ is semistable if and only if there exists no proper subspace $S(0) \subsetneq V(0)$ such that $S(0)$ is invariant for $x$ and $\operatorname{Im} i \subset S(0)$.

(ii) When $c>0,(x, z)$ is semistable if and only if there exists no nonzero subspace $0 \neq S(0) \subset V(0)$ such that $S(0)$ is invariant for $x$ and $S(0) \subset \operatorname{Ker} j$.

(iii) When $c=0, x$ is stable if and only if there exists no nonzero proper subspace $0 \neq S(0) \subsetneq V(0)$ such that $S(0)$ is invariant for $x$ and $\operatorname{Im} i \subset S(0) \subset \operatorname{Ker} j$.

This is because $u_{L} S(-l)=S(0), u_{R} S(l)=S(0)$. The above condition coincides with the (semi)stability condition of the quiver variety $\left\{\left[B_{1}, B_{2}\right]+i j=0\right\} / /{ }_{\chi} G L$ (c.f. $[\mathbf{N a 2}]$ ).

Corollary 4.12. When $\zeta \neq 0, \mathcal{M}_{\zeta}(n, l)$ is isomorphic to the Hilbert scheme $\left(\mathbb{C}^{2}\right)^{[n]}$, and when $\zeta=0, \mathcal{M}_{0}(n, l)$ is isomorphic to the symmetric product $S^{n}\left(\mathbb{C}^{2}\right)$ as algebraic varieties.

Proof. This follows from Proposition 4.11 and [Na2].

REMARK 4.13. These isomorphisms do not preserve the metrics.

\subsection{Metrics of elemental bow varieties}

First we calculate the metric of $\mathcal{M}_{0}(1, l)$. This is also mentioned by Cherkis [C1, §3.2]. In this case we have $B_{-+}, B_{+-}, i, j \in \mathbb{C}, T_{k}^{A} \in \Gamma\left(\mathcal{I}_{A}, \sqrt{-1} \mathbb{R}\right)$, and the moment map is given as follows:

$$
\begin{aligned}
\mu_{\mathbb{R}}= \begin{cases}\frac{-\sqrt{-1}}{2}\left(B_{-+} B_{-+}^{*}-B_{+-}^{*} B_{+-}\right)+T_{1}^{L}(-l) & \text { at } s=-l \\
\frac{d}{d s} T_{1}^{A} & \text { at } s \in(-l, 0),(0, l) \\
\frac{-\sqrt{-1}}{2}\left(i i^{*}-j^{*} j\right)-T_{1}^{L}(0)+T_{1}^{R}(0) & \text { at } s=0 \\
\frac{-\sqrt{-1}}{2}\left(B_{+-} B_{+-}^{*}-B_{-+}^{*} B_{-+}\right)-T_{1}^{R}(l) & \text { at } s=l,\end{cases} \\
\mu_{\mathbb{C}}= \begin{cases}B_{-+} B_{+-}+\left(T_{2}^{L}+\sqrt{-1} T_{3}^{L}\right)(-l) & \text { at } s=-l \\
\frac{d}{d s}\left(T_{2}^{A}+\sqrt{-1} T_{3}^{A}\right) & \text { at } s \in(-l, 0),(0, l) \\
\frac{\sqrt{-1}}{2} i j-\left(T_{2}^{L}+\sqrt{-1} T_{3}^{L}\right)(0)+\left(T_{2}^{R}+\sqrt{-1} T_{3}^{R}\right)(0) & \text { at } s=0 \\
-B_{+-} B_{-+}-\left(T_{2}^{R}+\sqrt{-1} T_{3}^{R}\right)(l) & \text { at } s=l .\end{cases}
\end{aligned}
$$

The equation $\mu=0$ means $T_{1}^{L} \equiv T_{1}^{R} \equiv \frac{\sqrt{-1}}{2}\left(\left|B_{-+}\right|^{2}-\left|B_{+-}\right|^{2}\right), T_{2}^{L}+\sqrt{-1} T_{3}^{L} \equiv T_{2}^{R}+$ $\sqrt{-1} T_{3}^{R} \equiv B_{+-} B_{-+}$, and $i=j=0$. Put $t_{k}=-\sqrt{-1} T_{k}^{A}, b_{1}=B_{-+}$and $b_{2}=B_{+-}$. We 
apply Proposition 3.13 for the interval $[-l, l]$, then we can conclude

$$
\begin{aligned}
\mathcal{M}_{0}(1, l)=\left\{\left(b_{1}, b_{2}, t_{0}, t_{k}\right) \in\right. & \mathbb{C} \times \mathbb{C} \times\left(\mathbb{R} /\left(l^{-1} \pi\right) \mathbb{Z}\right) \times \mathbb{R}^{3} \\
& \left.\left.|| b_{1}\right|^{2}-\left|b_{2}\right|^{2}=2 t_{1}, b_{1} b_{2}=\sqrt{-1} t_{2}-t_{3}\right\} / S^{1},
\end{aligned}
$$

where $S^{1}=\mathbb{R} / \mathbb{Z}=\{\phi\}$ acts as

$$
e^{\pi \sqrt{-1} \phi s / l}:\left(b_{1}, b_{2}, t_{0}, t_{k}\right) \mapsto\left(e^{-2 \pi \sqrt{-1} \phi} b_{1}, e^{2 \pi \sqrt{-1} \phi} b_{2},\left[t_{0}-l^{-1} \pi \phi\right], t_{k}\right) .
$$

The metric of the whole space $\left(\mathbb{R} /\left(l^{-1} \pi\right) \mathbb{Z}\right) \times \mathbb{R}^{3} \times \mathbb{C} \times \mathbb{C}$ is given by

$$
d s^{2}=d b_{1} d \bar{b}_{1}+d b_{2} d \bar{b}_{2}+2 l\left(d t_{0}^{2}+d t_{1}^{2}+d t_{2}^{2}+d t_{3}^{2}\right) .
$$

On the other hand, the Hopf fibration $\mathbb{C}^{2} \rightarrow \mathbb{R} \times \mathbb{C}$ is given by $\left(z_{1}, z_{2}\right) \mapsto\left(\left|z_{1}\right|^{2}-\right.$ $\left.\left|z_{2}\right|^{2}, 2 z_{1} z_{2}\right)$. If we put $\left(2 x_{1}, 2 \sqrt{-1} x_{2}-2 x_{3}\right)=\left(\left|z_{1}\right|^{2}-\left|z_{2}\right|^{2}, 2 z_{1} z_{2}\right)$, then $\left(x_{1}, x_{2}, x_{3}, \theta\right)$ becomes a new coordinate of $\mathbb{C}^{2}$, where $\theta$ is the coordinate of the $S^{1}$-fibre of the fibration. We have $d z_{1} d \bar{z}_{1}+d z_{2} d \bar{z}_{2}=1 /|x| d x^{2}+|x|(d \theta+\omega)^{2}$, where $d \omega=* d \frac{1}{|x|}$, * denotes the Hodge operator for $\mathbb{R}^{3}$. The $S^{1}$-action on $\{\theta\}$ is given by $\theta \mapsto \theta-2 \pi \sqrt{-1} \phi$. By using the coordinate $\left(x_{1}, x_{2}, x_{3}, \theta\right)$, the above equations are written as $t_{k}=x_{k}$, and the restricted metric is given by

$$
\begin{aligned}
d s^{2} & =\frac{1}{|x|} d x^{2}+|x|(d \theta+\omega)^{2}+2 l\left(d t_{0}^{2}+d x_{k}^{2}\right) \\
& =\left(2 l+\frac{1}{|x|}\right) d x^{2}+|x|(d \theta+\omega)^{2}+2 l d t_{0}^{2} .
\end{aligned}
$$

And $\theta-2 l \sqrt{-1} t_{0}$ is invariant by the $S^{1}$-action, we put $\psi=\theta-2 l \sqrt{-1} t_{0}$. By using $\psi$, we have

$$
d s^{2}=\left(2 l+\frac{1}{|x|}\right) d x^{2}+\left(2 l+\frac{1}{|x|}\right)^{-1}(d \psi+\omega)^{2} .
$$

This coincides with Taub-NUT metric $g_{\mathrm{TN}}(4.2)$, so $\mathcal{M}_{0}(1, l)$ is regarded as Taub-NUT space. Conversely, we can consider that the hyper-Kähler structure of Taub-NUT space is defined by that of $\mathcal{M}_{0}(1, l)$. In this paper TN denotes Taub-NUT space. This metric converges to the normal metric of $\mathbb{C}^{2}$ as $l \rightarrow 0$, and this corresponds to the behaviour of the diagram:

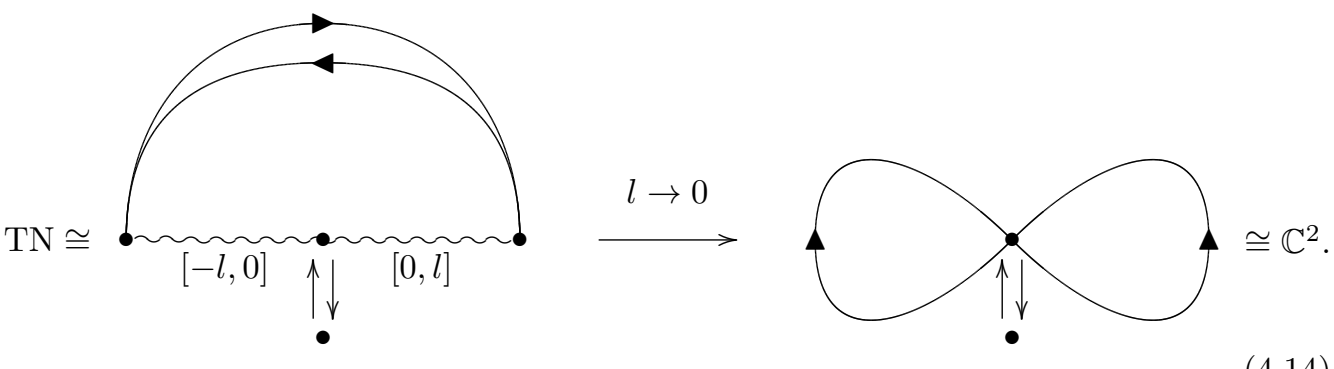

We note the relation between Taub-NUT space and $\mathbb{C}^{2}$ without taking the limit $l \rightarrow 0$. As $\S 4.1$ we apply Kronheimer's theorem to $\mathcal{M}_{0}(1, l)$, and by Proposition 3.13 we have

$$
\begin{array}{ll}
u_{L}=\exp \left\{-l\left(\sqrt{-1} t_{0}-t_{1}\right)\right\}, & \eta_{L}=\left(\sqrt{-1} t_{2}-t_{3}\right) / 2, \\
u_{R}=\exp \left\{l\left(\sqrt{-1} t_{0}-t_{1}\right)\right\}, & \eta_{R}=\left(\sqrt{-1} t_{2}-t_{3}\right) / 2 .
\end{array}
$$


Put $u:=u_{L} u_{R}^{-1}=\exp \left\{-2 l\left(\sqrt{-1} t_{0}-t_{1}\right)\right\}, \eta:=\eta_{L}=\eta_{R}=\left(\sqrt{-1} t_{2}-t_{3}\right) / 2$. Then we have

$$
\begin{aligned}
\mathrm{TN} & =\left\{\left.\left(b_{1}, b_{2}, t_{0}, t_{k}\right)|| b_{1}\right|^{2}-\left|b_{2}\right|^{2}=2 t_{1}, b_{1} b_{2}=\sqrt{-1} t_{2}-t_{3}\right\} / S^{1} \\
& =\left\{\left.\left(b_{1}, b_{2}, u, \eta\right)|| b_{1}\right|^{2}-\left|b_{2}\right|^{2}=l^{-1} \operatorname{Re} \log u, 2 b_{1} b_{2}=\eta\right\} / S^{1}
\end{aligned}
$$

And the Kähler forms of Taub-NUT space are written as

$$
\begin{aligned}
\omega_{I} & =\frac{\sqrt{-1}}{2}\left(d b_{1} \wedge d \bar{b}_{1}+d b_{2} \wedge d \bar{b}_{2}\right)+2 l\left(d t_{0} \wedge d t_{1}+d t_{2} \wedge d t_{3}\right) \\
& =\frac{\sqrt{-1}}{2}\left(d b_{1} \wedge d \bar{b}_{1}+d b_{2} \wedge d \bar{b}_{2}\right)+\frac{\sqrt{-1}}{4 l} d \log u \wedge d \log \bar{u}+\frac{\sqrt{-1} l}{4} d \eta \wedge d \bar{\eta}, \\
\omega_{J}+\sqrt{-1} \omega_{K} & =d b_{1} \wedge d b_{2}+2 l\left(\sqrt{-1} d t_{0}-d t_{1}\right) \wedge\left(-\sqrt{-1} d t_{2}+d t_{3}\right) \\
& =d b_{1} \wedge d b_{2}+2 d \log u \wedge d \eta .
\end{aligned}
$$

Furthermore by Proposition 4.7, we have the following isomorphism as algebraic varieties:

$$
\Phi:\left\{\left(b_{1}, b_{2}, u, \eta\right) \mid \begin{array}{c}
\left|b_{1}\right|^{2}-\left|b_{2}\right|^{2}=l^{-1} \operatorname{Re} \log u, \\
2 b_{1} b_{2}=\eta
\end{array}\right\} / S^{1} \stackrel{\cong}{\longrightarrow}\left\{\left(b_{1}, b_{2}, u, \eta\right) \mid b_{1} b_{2}=2 \eta\right\} / \mathbb{C}^{*} .
$$

$\Phi$ is given by the inclusion map, and $\Phi^{-1}$ is given by the action of $v \in \mathbb{C}^{*}$ such that $\left(v^{-1} b_{1}, v b_{2}, v u, \eta\right)$ satisfies $\mu_{\mathbb{R}}=0$, i.e. $v^{-2}\left|b_{1}\right|^{2}-v^{2}\left|b_{2}\right|^{2}=l^{-1} \operatorname{Re} \log (v u)$. We define $\mathrm{TN}_{\mathbb{C}}:=\left\{\left(b_{1}, b_{2}, u, \eta\right) \mid b_{1} b_{2}=2 \eta\right\} / \mathbb{C}^{*}$.

Proposition 4.16. Taub-NUT space is holomorphic symplectomorphic to $\mathbb{C}^{2}$. And the morphism is explicitly given by

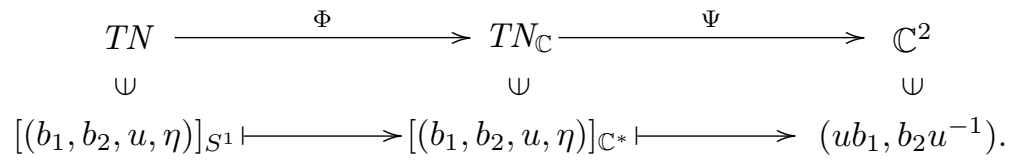

Proof. This is the conclusion of Proposition 4.11 in the case of $n=1$. And we have

$$
\begin{aligned}
d z_{1} \wedge d z_{2} & =d\left(u b_{1}\right) \wedge d\left(b_{2} u^{-1}\right) \\
& =d b_{1} \wedge d b_{2}-b_{1} d b_{2} \wedge u^{-1} d u+b_{2} d b_{1} \wedge u d\left(u^{-1}\right) \\
& =d b_{1} \wedge d b_{2}+2 u^{-1} d u \wedge d \eta
\end{aligned}
$$

REMARK 4.17. $\Psi^{-1}$ is given by $\Psi^{-1}\left(z_{1}, z_{2}\right)=\left[\left(z_{1}, z_{2}, 1, z_{1} z_{2} / 2\right)\right]_{\mathbb{C}^{*}}$.

Next we consider $\mathcal{M}_{0}(n, l)$ in the case of $n \geq 1$.

Theorem 4.18. (i) As algebraic varieties, $\mathcal{M}_{0}(n, l)$ is isomorphic to $S^{n}\left(\mathbb{C}^{2}\right)$ and the holomorphic symplectic form is preserved.

(ii) As hyper-Kähler manifolds, $\mathcal{M}_{0}(n, l)$ is isomorphic to $S^{n}(\mathrm{TN})$.

Proof. (i) follows from Corollary 4.12 and Proposition 4.16. Let us prove (ii). We check how $(B, i, j, T) \in \mu^{-1}(0)$ is described.

Since there exists the isomorphism $\mathcal{M}_{0}(n, l) \cong S^{n}\left(\mathbb{C}^{2}\right)$ given by Proposition 4.11 and 


\section{YUUYA TAKAYAMA}

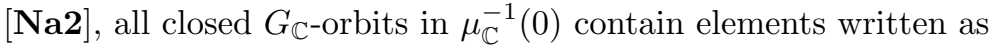

$$
u_{L} B_{-+} u_{R}^{-1}=B_{1}=\left(\begin{array}{ccc}
b_{1}^{1.1} & & 0 \\
0 & \ddots & b_{1}^{n . n}
\end{array}\right), u_{R} B_{+-} u_{L}^{-1}=B_{2}=\left(\begin{array}{ccc}
b_{2}^{1.1} & & 0 \\
0 & \ddots & b_{2}^{n . n}
\end{array}\right), i=j=0 .
$$

Furthermore, by using the action of the subgroup $\left\{\left(g_{-}\right.\right.$, id, $\left.\left.g_{+}\right)\right\} \subset G_{\mathbb{C}}$, we can regard $u_{L}=u_{R}=\mathrm{id}_{n}$, and then we can assume

$$
\left(B_{-+}, B_{+-}, u_{A}, \eta_{A}\right)=\left(\left(\begin{array}{ccc}
b_{1}^{1.1} & & 0 \\
0 & \ddots & b_{1}^{n . n}
\end{array}\right),\left(\begin{array}{ccc}
b_{2}^{1.1} & & 0 \\
0 & \ddots & b_{2}^{n . n}
\end{array}\right), \operatorname{id}_{n},\left(\begin{array}{ccc}
\eta_{A}^{1.1} \cdot & 0 \\
0 & \ddots & \eta_{A}^{n . n}
\end{array}\right)\right),
$$

where $b_{1}^{\bullet} b_{2}^{\bullet}=2 \eta_{L}^{\bullet}=2 \eta_{R}^{\bullet}$ holds (c.f. (4.6)).

Now we consider the action of the subgroup

$$
\left\{\left(\text { id, }\left(\begin{array}{cc|c}
h^{1.1} & 0 \\
& \ddots & 0 \\
0 & & h^{n . n}
\end{array}\right), \text { id }\right) \in G_{\mathbb{C}} \mid h^{\bullet} \in \mathbb{C}^{*}\right\} .
$$

Note that this action preserves the description of (4.19). Each $\left[\left(b_{1}^{\bullet}, b_{2}^{\bullet}, 1, \eta^{\bullet}\right)\right]_{\mathbb{C}^{*}}$ determines a point in $\mathrm{TN}_{\mathbb{C}}$, and each $\Psi^{-1}\left(\left[\left(b_{1}^{\bullet}, b_{2}^{\bullet}, 1, \eta^{\bullet}\right)\right]_{\mathbb{C}^{*}}\right)$ determines a point in TN. Thus all the points in $\mu_{\mathbb{R}}^{-1}(0) \cap \mu_{\mathbb{C}}^{-1}(0) / G$ are represented as

$$
\left(B_{-+}, B_{+-}, u, \eta\right)=\left(\left(\begin{array}{ccc}
b_{1}^{1.1} & & 0 \\
& \ddots & 0 \\
0 & & b_{1}^{n . n}
\end{array}\right),\left(\begin{array}{ccc}
b_{2}^{1.1} & & 0 \\
0 & \ddots & b_{2}^{n . n}
\end{array}\right),\left(\begin{array}{ccc}
u^{1.1} & & 0 \\
& \ddots & \\
0 & & u^{n . n}
\end{array}\right),\left(\begin{array}{ccc}
\eta^{1.1} & 0 \\
0 & \ddots & \eta^{n . n}
\end{array}\right)\right),
$$

where each $\left(b_{1}^{\bullet}, b_{2}^{\bullet}, u^{\bullet}, \eta^{\bullet}\right) \in \mathrm{TN}$.

Therefore we get an isomorphism $\mathcal{M}_{0}(n, l) \cong(\mathrm{TN})^{n} / \mathfrak{S}_{n}$.

REMARK 4.21. As a GIT quotient, we can take a representative $x=\left(B_{-+}, B_{+_{-}}, i, j\right.$, $\left.u_{A}, \eta_{A}\right)$ of $[x]_{G_{\mathbb{C}}} \in \mathcal{M}_{0}(n, l)$ as

$$
x=\left(\left(\begin{array}{ccc}
b_{1}^{1.1} & & 0 \\
0 & \ddots & b_{1}^{n . n}
\end{array}\right),\left(\begin{array}{ccc}
b_{2}^{1.1} & & 0 \\
& \ddots & \\
0 & & b_{2}^{n . n}
\end{array}\right),\left(\begin{array}{c}
1 \\
\vdots \\
1
\end{array}\right), 0,\left(\begin{array}{ccc}
u_{A}^{1.1} \cdot & & 0 \\
0 & & u_{A}^{n . n}
\end{array}\right),\left(\begin{array}{ccc}
\eta_{A}^{1.1} \cdot & 0 \\
0 & \ddots & \eta_{A}^{n . n}
\end{array}\right)\right) .
$$

This is because the closure of $G_{\mathbb{C}} \cdot(B, i, 0, u, \eta)$ contains the closed orbit $G_{\mathbb{C}} \cdot(B, 0,0, u, \eta)$ (see also $\left[\mathbf{N a 2}\right.$, Proposition 2.8.]). Notice that unlike on the representative (4.20), $G_{\mathbb{C}}$ acts freely on this representative.

\section{Construction of other bow varieties}

Let $\Gamma$ be a finite subgroup of $S U(2), Q$ be its canonical 2-dimensional representation, and $R$ be its regular representation. At [Kr2], Kronheimer showed

$$
\mathbb{C}^{2} / \Gamma \cong X_{0}=\left\{(\alpha, \beta) \in(Q \otimes \operatorname{End}(R))^{\Gamma} \mid \mu(\alpha, \beta)=0\right\} / U(R)^{\Gamma},
$$

and $X_{0}$ is a quiver variety corresponding to the extended Dynkin diagram of $\Gamma$.

In this section, we find out bow varieties which are isomorphic to Taub-NUT/ $\Gamma$ as hyper-Kähler manifolds. 


\section{5·1. Automorphisms of Taub-NUT space}

In this subsection, we first discuss about the automorphisms of Taub-NUT space. Then we consider divided Taub-NUT space by finite groups.

TheOREm 5.1. As a hyper-Kähler manifold, the automorphism group preserving the origin of Taub-NUT space is the semi-direct product $\mathbb{Z}_{2} \ltimes S^{1}$ generated by

$$
\begin{aligned}
\omega_{\zeta}:\left[\left(b_{1}, b_{2}, u, \eta\right)\right]_{S^{1}} & \mapsto\left[\left(\zeta b_{1}, \zeta^{-1} b_{2}, u, \eta\right)\right]_{S^{1}}, \\
\iota:\left[\left(b_{1}, b_{2}, u, \eta\right)\right]_{S^{1}} & \mapsto\left[\left(-b_{2}, b_{1}, u^{-1},-\eta\right)\right]_{S^{1}},
\end{aligned}
$$

where $\zeta \in \mathbb{C},|\zeta|=1$ and the origin is $[(0,0,1,0)]_{S^{1}} \in T N$.

We consider what actions preserve the Kähler form $\omega_{I}$ and the holomorphic symplectic form $\omega_{J}+\sqrt{-1} \omega_{K}$. By Proposition 4.16, Taub-NUT space is holomorphic symplectomorphic to $\mathbb{C}^{2}$, and the holomorphic symplectic form is written as $d z_{1} \wedge d z_{2}$. So all the actions which preserve the holomorphic symplectic form are described as $\Phi^{-1} \circ \Psi^{-1} \circ f \circ \Psi \circ \Phi$ by using a biholomorphism $f(\Phi$ and $\Psi$ are given at Proposition 4.16):

$$
f: \mathbb{C}^{2} \rightarrow \mathbb{C}^{2}, f\left(z_{1}, z_{2}\right)=\left(f_{1}\left(z_{1}, z_{2}\right), f_{2}\left(z_{1}, z_{2}\right)\right) .
$$

Take $\left[\left(b_{1}, b_{2}, u, \eta\right)\right]_{S^{1}} \in \mathrm{TN}$ and fix the $S^{1}$-action as $u \in \mathbb{R}$ i.e. $u=\exp \left\{l\left|b_{1}\right|^{2}-l\left|b_{2}\right|^{2}\right\}$. Then the action is written as follows:

$$
\begin{aligned}
& \tilde{f}:=\Phi^{-1} \circ \Psi^{-1} \circ f \circ \Psi \circ \Phi, \\
& \tilde{f}\left(b_{1}, b_{2}, u, b_{1} b_{2} / 2\right)=\left(e^{-r} f_{1}\left(u b_{1}, u^{-1} b_{2}\right), e^{r} f_{2}\left(u b_{1}, u^{-1} b_{2}\right), e^{r}, f_{1} f_{2} / 2\right),
\end{aligned}
$$

where $r \in \mathbb{R}$ satisfies

$$
e^{-2 r}\left|f_{1}\right|^{2}-e^{2 r}\left|f_{2}\right|^{2}=\frac{r}{l}
$$

Needless to say, $r$ depends on $b_{1}, b_{1}, u, f_{1}$ and $f_{2}$.

From now, we consider when this action $\tilde{f}$ preserves $\omega_{I}$. Here, fixing the $S^{1}$-action, we have

$$
-2 \sqrt{-1} \omega_{I}=d b_{1} \wedge d \bar{b}_{1}+d b_{2} \wedge d \bar{b}_{2}+2 l d\left(b_{1} b_{2}\right) \wedge d \overline{\left(b_{1} b_{2}\right)}
$$

Put

$$
\begin{aligned}
& f_{1}\left(z_{1}, z_{2}\right)=p_{10} z_{1}+p_{01} z_{2}+h_{1}\left(z_{1}, z_{2}\right), \\
& f_{2}\left(z_{1}, z_{2}\right)=q_{10} z_{1}+q_{01} z_{2}+h_{2}\left(z_{1}, z_{2}\right),
\end{aligned}
$$

where $h_{k}$ are holomorphic and $h(0,0)=0, \frac{\partial h}{\partial z}(0,0)=0$. Since $\left(f_{1}, f_{2}\right)$ preserves $d z_{1} \wedge d z_{2}$, we get

$$
f_{1,1} f_{2,2}-f_{1,2} f_{2,1}=1 \text {. }
$$

Here, for example, $f_{1,2}$ denotes $\frac{\partial f_{1}}{\partial z_{2}}$.

The exterior derivative of $f_{k}\left(u b_{1}, u^{-1} b_{1}\right)$ and $r$ is written as

$$
\begin{aligned}
d\left(f_{1}\left(u b_{1}, u^{-1} b_{2}\right)\right) & =f_{1,1}\left(u b_{1}, u^{-1} b_{2}\right) d\left(u b_{1}\right)+f_{1,2}\left(u b_{1}, u^{-1} b_{2}\right) d\left(u^{-1} b_{2}\right), \\
d\left(f_{2}\left(u b_{1}, u^{-1} b_{2}\right)\right) & =f_{2,1}\left(u b_{1}, u^{-1} b_{2}\right) d\left(u b_{1}\right)+f_{2,2}\left(u b_{1}, u^{-1} b_{2}\right) d\left(u^{-1} b_{2}\right), \\
d r & =\frac{l\left\{e^{-2 r}\left(d f_{1} \bar{f}_{1}+d \bar{f}_{1} f_{1}\right)-e^{2 r}\left(d f_{2} \bar{f}_{2}+d \bar{f}_{2} f_{2}\right)\right\}}{2 l\left(e^{-2 r}\left|f_{1}\right|^{2}+e^{2 r}\left|f_{2}\right|^{2}\right)+1} .
\end{aligned}
$$


The induced Kähler form by $\tilde{f}$ is

$$
\begin{aligned}
-2 \sqrt{-1} \tilde{f}^{*} \omega_{I}= & d\left(e^{-r} f_{1}\right) \wedge d\left(e^{-r} \bar{f}_{1}\right)+d\left(e^{r} f_{2}\right) \wedge d\left(e^{r} \bar{f}_{2}\right)+2 l d\left(f_{1} f_{2}\right) \wedge d \overline{\left(f_{1} f_{2}\right)} \\
= & e^{-2 r} d f_{1} \wedge d \bar{f}_{1}+e^{2 r} d f_{2} \wedge d \bar{f}_{2}+2 l d\left(f_{1} f_{2}\right) \wedge d \overline{\left(f_{1} f_{2}\right)} \\
& \quad+d r \wedge\left\{e^{-2 r}\left(-f_{1} d \bar{f}_{1}+\bar{f}_{1} d f_{1}\right)+e^{2 r}\left(f_{2} d \bar{f}_{2}-\bar{f}_{2} d f_{2}\right)\right\} \\
= & \frac{1}{2 l\left(e^{-2 r}\left|f_{1}\right|^{2}+e^{2 r}\left|f_{2}\right|^{2}\right)+1}\left\{e^{-2 r} d f_{1} \wedge d \bar{f}_{1}+e^{2 r} d f_{2} \wedge d \bar{f}_{2}\right. \\
& \left.\quad+4 l\left(l e^{-2 r}\left|f_{1}\right|^{2}+l e^{2 r}\left|f_{2}\right|^{2}+1\right) d\left(f_{1} f_{2}\right) \wedge d \overline{\left(f_{1} f_{2}\right)}\right\} .
\end{aligned}
$$

1.

Considering $\tilde{f}^{*} \omega_{I}=\omega_{I}$ at the origin, we have $q_{10}=-\bar{p}_{01}, q_{01}=\bar{p}_{10}$ and $\left|p_{10}\right|^{2}+\left|p_{01}\right|^{2}=$

LEMmA 5.4. When $\tilde{f}^{*} \omega_{I}=\omega_{I}$, we have $h_{1}=h_{2}=0$.

Proof. From $\tilde{f}^{*} \omega_{I}$ we pick up 2-forms whose coefficients are holomorphic functions. Expanding $\tilde{f}^{*} \omega_{I}$ with respect to $b_{1}, \bar{b}_{1}, b_{2}$ and $\bar{b}_{2}$ around the origin, then we collect 2forms whose coefficients consist of products of $b_{1}$ and $b_{2}$ (i.e. we ignore terms containing $\bar{b}_{1}$ and $\left.\bar{b}_{2}\right)$.

First we consider the expansion of $u$ and $r$. We have

$$
\begin{aligned}
u & =e^{l\left|b_{1}\right|^{2}-l\left|b_{2}\right|^{2}} \\
& =1+\left(l\left|b_{1}\right|^{2}-l\left|b_{2}\right|^{2}\right)+\frac{\left(l\left|b_{1}\right|^{2}-l\left|b_{2}\right|^{2}\right)^{2}}{2}+O\left(|b|^{4}\right),
\end{aligned}
$$

and since $r$ is analytic around the origin, from (5.2), we have

$$
\begin{aligned}
r & =\left(l\left|p_{10}\right|^{2}-l\left|q_{10}\right|^{2}\right)\left|b_{1}\right|^{2}+\left(l\left|p_{01}\right|^{2}-l\left|q_{01}\right|^{2}\right)\left|b_{2}\right|^{2}+O\left(|b|^{3}\right) \\
& =\left(l\left|p_{10}\right|^{2}-l\left|p_{01}\right|^{2}\right)\left(\left|b_{1}\right|^{2}-\left|b_{2}\right|^{2}\right)+O\left(|b|^{3}\right) .
\end{aligned}
$$

Especially the holomorphic parts of $u$ and $r$ are respectively 1 and 0 . Then we find that the holomorphic parts of $f_{1}\left(u b_{1}, u^{-1} b_{2}\right), \overline{f_{1}\left(u b_{1}, u^{-1} b_{2}\right)}, f_{1,1}\left(u b_{1}, u^{-1} b_{2}\right)$ and $\overline{f_{1,1}\left(u b_{1}, u^{-1} b_{2}\right)}$ are respectively $f_{1}\left(b_{1}, b_{2}\right), 0, f_{1,1}\left(b_{1}, b_{2}\right)$ and $\bar{p}_{10}$.

Therefore we only consider the term $d f_{1} \wedge d \bar{f}_{1}+d f_{2} \wedge d \bar{f}_{2}$. And we have 1-forms whose coefficients are holomorphic:

$$
\begin{aligned}
d\left(f_{1}\left(u b_{1}, u^{-1} b_{2}\right)\right) \rightsquigarrow & f_{1,1}\left(b_{1}, b_{2}\right)\left\{\left(l b_{1} d \bar{b}_{1}-l b_{2} d \bar{b}_{2}\right) b_{1}+d b_{1}\right\} \\
& +f_{1,2}\left(b_{1}, b_{2}\right)\left\{-\left(l b_{1} d \bar{b}_{1}-l b_{2} d \bar{b}_{2}\right) b_{2}+d b_{2}\right\}, \\
d\left(f_{2}\left(u b_{1}, u^{-1} b_{2}\right)\right) \rightsquigarrow & f_{2,1}\left(b_{1}, b_{2}\right)\left\{\left(l b_{1} d \bar{b}_{1}-l b_{2} d \bar{b}_{2}\right) b_{1}+d b_{1}\right\} \\
& +f_{2,2}\left(b_{1}, b_{2}\right)\left\{-\left(l b_{1} d \bar{b}_{1}-l b_{2} d \bar{b}_{2}\right) b_{2}+d b_{2}\right\}, \\
\overline{d\left(f_{1}\left(u b_{1}, u^{-1} b_{2}\right)\right)} \rightsquigarrow & \bar{p}_{10} d \bar{b}_{1}+\bar{p}_{01} d \bar{b}_{2}, \\
d \overline{\left(f_{2}\left(u b_{1}, u^{-1} b_{2}\right)\right)} \rightsquigarrow & \bar{q}_{10} d \bar{b}_{1}+\bar{q}_{01} d \bar{b}_{2} .
\end{aligned}
$$

Then we get

$$
\begin{aligned}
& d f_{1} \wedge d \bar{f}_{1} \rightsquigarrow l\left(f_{1,1} b_{1}-f_{1,2} b_{2}\right)\left(\bar{p}_{10} b_{2}+\bar{p}_{01} b_{1}\right) d \bar{b}_{1} \wedge d \bar{b}_{2} \\
& +f_{1,1} \bar{p}_{10} d b_{1} \wedge d \bar{b}_{1}+f_{1,1} \bar{p}_{01} d b_{1} \wedge d \bar{b}_{2}+f_{1,2} \bar{p}_{01} d b_{2} \wedge d \bar{b}_{2}+f_{1,2} \bar{p}_{10} d b_{2} \wedge d \bar{b}_{1}, \\
& d f_{2} \wedge d \bar{f}_{2} \rightsquigarrow l\left(f_{2,1} b_{1}-f_{2,2} b_{2}\right)\left(\bar{q}_{10} b_{2}+\bar{q}_{01} b_{1}\right) d \bar{b}_{1} \wedge d \bar{b}_{2} \\
& +f_{2,1} \bar{q}_{10} d b_{1} \wedge d \bar{b}_{1}+f_{2,1} \bar{q}_{01} d b_{1} \wedge d \bar{b}_{2}+f_{2,2} \bar{q}_{01} d b_{2} \wedge d \bar{b}_{2}+f_{2,2} \bar{q}_{10} d b_{2} \wedge d \bar{b}_{1} .
\end{aligned}
$$


On the other hand, the holomorphic part of $-2 \sqrt{-1} \omega_{I}$ is $d b_{1} \wedge d \bar{b}_{1}+d b_{2} \wedge d \bar{b}_{2}$. Therefore when $\tilde{f}^{*} \omega_{I}=\omega_{I}$, we have

$$
\begin{aligned}
& f_{1,1} \bar{p}_{01}+f_{2,1} \bar{q}_{01}=0, \\
& f_{1,2} \bar{p}_{10}+f_{2,2} \bar{q}_{10}=0 .
\end{aligned}
$$

Comparing them with (5.3), we have $f_{1}=p_{10} z_{1}+p_{01} z_{2}$ and $f_{2}=-\bar{p}_{01} z_{1}+\bar{p}_{10} z_{2}$.

LEMMA 5.5. In addition to Lemma 5.4, we have $p_{10} p_{01}=0$.

Proof. Put $P=\left(0, \epsilon, e^{-l \epsilon^{2}}, 0\right),(\epsilon \in \mathbb{R})$. We calculate how $\left.\tilde{f}^{*} \omega_{I}\right|_{P}$ and $\left.\omega_{I}\right|_{P}$ vary as $\epsilon$ varies. Especially we pick up the coefficient of $d b_{1} \wedge d \bar{b}_{1}$-term.

Since $\tilde{f}(P)=\left(e^{-r} p_{01} e^{l \epsilon^{2}} \epsilon, e^{r} p_{10}^{-} e^{l \epsilon^{2}} \epsilon, e^{r}, p_{01} p_{10}^{-} e^{2 l \epsilon^{2}} \epsilon^{2} / 2\right)$, we have

$$
\begin{aligned}
&-\left.2 \sqrt{-1} \omega_{I}\left(\frac{\partial}{\partial b_{1}}, \frac{\partial}{\partial \bar{b}_{1}}\right)\right|_{P}=1+2 l \epsilon^{2}, \\
& d f_{1}\left.\wedge d \bar{f}_{1}\left(\frac{\partial}{\partial b_{1}}, \frac{\partial}{\partial \bar{b}_{1}}\right)\right|_{P}=e^{-2 l \epsilon^{2}}\left|p_{10}\right|^{2}, \\
&\left.d f_{2} \wedge d \bar{f}_{2}\left(\frac{\partial}{\partial b_{1}}, \frac{\partial}{\partial \bar{b}_{1}}\right)\right|_{P}=e^{-2 l \epsilon^{2}}\left|p_{01}\right|^{2}, \\
&\left.d\left(f_{1} f_{2}\right) \wedge d \overline{\left(f_{1} f_{2}\right)}\left(\frac{\partial}{\partial b_{1}}, \frac{\partial}{\partial \bar{b}_{1}}\right)\right|_{P}=\left(\left|p_{10}\right|^{2}-\left|p_{01}\right|^{2}\right)^{2} \epsilon^{2},
\end{aligned}
$$

so if $\tilde{f}^{*} \omega_{I}=\omega_{I}$, we get

$$
\begin{aligned}
& \left\{2 l\left(e^{-2 r}\left|f_{1}\right|^{2}+e^{2 r}\left|f_{2}\right|^{2}\right)+1\right\}\left(1+2 l \epsilon^{2}\right) \\
& \quad=e^{-2 r} e^{-2 l \epsilon^{2}}\left|p_{10}\right|^{2}+e^{2 r} e^{-2 l \epsilon^{2}}\left|p_{01}\right|^{2}+4 l\left(l e^{-2 r}\left|f_{1}\right|^{2}+l e^{2 r}\left|f_{2}\right|^{2}+1\right)\left(\left|p_{10}\right|^{2}-\left|p_{01}\right|^{2}\right)^{2} \epsilon^{2} .
\end{aligned}
$$

Now we expand them by $\epsilon$ around $\epsilon=0$. Since we have

$$
\begin{aligned}
u & =1-l \epsilon^{2}+O\left(\epsilon^{4}\right), & r & =l\left(\left|p_{01}\right|^{2}-\left|p_{10}\right|^{2}\right) \epsilon^{2}+O\left(\epsilon^{3}\right), \\
\left|f_{1}\left(0, e^{l \epsilon^{2}} \epsilon\right)\right| & =\left|p_{01}\right| \epsilon+O\left(\epsilon^{2}\right), & \left|f_{2}\left(0, e^{l \epsilon^{2}} \epsilon\right)\right| & =\left|p_{10}\right| \epsilon+O\left(\epsilon^{2}\right),
\end{aligned}
$$

we get

$$
\begin{aligned}
& \left\{2 l\left|p_{01}\right|^{2} \epsilon^{2}+2 l\left|p_{10}\right|^{2} \epsilon^{2}+1\right\}\left(1+2 l \epsilon^{2}\right)+O\left(\epsilon^{3}\right) \\
& =\left(1-2 l \epsilon^{2}\right)\left(1-2 l\left(\left|p_{01}\right|^{2}-\left|p_{10}\right|^{2}\right) \epsilon^{2}\right)\left|p_{10}\right|^{2}+\left(1-2 l \epsilon^{2}\right)\left(1+2 l\left(\left|p_{01}\right|^{2}-\left|p_{10}\right|^{2}\right) \epsilon^{2}\right)\left|p_{01}\right|^{2} \\
& \quad+4 l\left(l\left|p_{01}\right|^{2} \epsilon^{2}+l\left|p_{10}\right|^{2} \epsilon^{2}+1\right)\left(\left|p_{10}\right|^{2}-\left|p_{01}\right|^{2}\right)^{2} \epsilon^{2}+O\left(\epsilon^{3}\right), \\
& \Leftrightarrow 1+4 l \epsilon^{2}+O\left(\epsilon^{3}\right)=1+4 l \epsilon^{2}-24 l \epsilon^{2}\left|p_{10} p_{01}\right|^{2}+O\left(\epsilon^{3}\right) .
\end{aligned}
$$

Therefore we have $p_{10} p_{01}=0$.

Proof of Theorem 5.1 By Lemma 5.4 and Lemma 5.5, if $\tilde{f}$ preserves the hyper-Kähler structure of Taub-NUT space and the origin, we have

$$
f\left(z_{1}, z_{2}\right)=\left(\begin{array}{cc}
p & 0 \\
0 & \bar{p}
\end{array}\right)\left(\begin{array}{l}
z_{1} \\
z_{2}
\end{array}\right),\left(\begin{array}{cc}
0 & q \\
-\bar{q} & 0
\end{array}\right)\left(\begin{array}{l}
z_{1} \\
z_{2}
\end{array}\right), \quad|p|=|q|=1 .
$$

The former leads to $\tilde{f}\left(b_{1}, b_{2}, u, \eta\right)=\left[\left(p b_{1}, \bar{p} b_{2}, u, \eta\right)\right]$ and the latter leads to $\tilde{f}\left(b_{1}, b_{2}, u, \eta\right)=$ $\left[\left(-\bar{q} b_{2}, q b_{1}, u^{-1}, \eta\right)\right]$. 


\section{YUUYA TAKAYAMA}

By Theorem 5.1, finite groups acting on Taub-NUT space which preserve the origin are generated by

$$
\begin{aligned}
\omega_{m}:\left[\left(b_{1}, b_{2}, u, \eta\right)\right]_{S^{1}} & \mapsto\left[\left(\zeta_{m} b_{1}, \zeta_{m}^{-1} b_{2}, u, \eta\right)\right]_{S^{1}}, \\
\iota:\left[\left(b_{1}, b_{2}, u, \eta\right)\right]_{S^{1}} & \mapsto\left[\left(-b_{2}, b_{1}, u^{-1},-\eta\right)\right]_{S^{1}},
\end{aligned}
$$

where $\zeta_{m}^{m}=1$. We define

$$
\begin{aligned}
& A_{n}=\left\langle\omega_{n+1}\right\rangle \quad(n \geq 1), \\
& D_{n}=\left\langle\omega_{2 n-4}, \iota\right\rangle \quad(n \geq 3) .
\end{aligned}
$$

In fact they correspond to the finite subgroups of $S U(2)$ by $\Phi \circ \Psi: \mathrm{TN} \rightarrow \mathbb{C}^{2}$.

Corollary 5.6. $E_{n} \subset S U(2)$ does not act on Taub-NUT space in such a way as to preserve the hyper-Kähler structure and the origin.

REMARK 5.7. As the subgroups of $S U(2), A_{3}$ and $D_{3}$ are identified because $\left(\begin{array}{cc}0 & 1 \\ -1 & 0\end{array}\right)$ and $\left(\begin{array}{cc}\sqrt{-1} & 0 \\ 0 & -\sqrt{-1}\end{array}\right)$ are conjugate. However as the actions on Taub-NUT space, $A_{3}$ and $D_{3}$ are different.

Let $\Gamma$ be $A_{n}$ or $D_{n}$. The $\Gamma$-action on Taub-NUT space induces it on $S^{n}(\mathrm{TN})$.

Proposition 5.8. Put $m=\sharp \Gamma$. $\left(S^{m}(T N)\right)^{\Gamma}$ and $T N / \Gamma$ are isomorphic as hyperKähler manifolds.

Proof. By definition of the symmetric product, $\Gamma$-fixed points in $S^{m}(\mathrm{TN})$ consist of $m$-points in Taub-NUT space all of which are in the same $\Gamma$-orbit. And these $\Gamma$-orbits correspond to elements of $\mathrm{TN} / \Gamma$.

\subsection{Decomposition of elemental bows}

In the last subsection, we constructed $\mathrm{TN} / \Gamma$ as $\Gamma$-fixed points. In this subsection, we introduce the way to construct $\mathrm{TN} / \Gamma$ by a hyper-Kähler quotient. To do this, we first consider the way to construct $\mathrm{TN} / \Gamma$ by an affine algebro-geometric quotient.

First we consider a general case. Let $M$ be an affine variety and $G$ be an algebraic group acting on $M$. Put $M^{\text {free }}=\{x \in M \mid g x \neq x$ for all $g \in G \backslash$ id $\}$. And suppose a finite group $\Gamma$ acts on $M$ in such a way as to commute with the $G$-action. Then the $\Gamma$-action descends to the affine algebro-geometric quotient $M / / 0 G$. When we distinguish these two actions, we describe the $G$-action as $g \cdot x$ and the $\Gamma$-action as $\gamma \star x$, for $x \in M, g \in G, \gamma \in \Gamma$.

Assume we can take a representative $x \in M^{\text {free }}$ for any $[x]_{G} \in\left(M / /{ }_{0} G\right)^{\Gamma}$. Then by the freeness, for any $\gamma \in \Gamma$ there exists unique $\rho_{x}(\gamma) \in G$ which satisfies $\gamma \star x=\rho_{x}(\gamma)^{-1} \cdot x$.

Lemma 5.9. This $\rho_{x}$ is a homomorphism from $\Gamma$ to $G$.

Proof. We get $\rho_{x}\left(\gamma_{1} \gamma_{2}\right)^{-1} \cdot x=\left(\gamma_{1} \gamma_{2}\right) \star x=\gamma_{1} \star\left(\rho_{x}\left(\gamma_{2}\right)^{-1} \cdot x\right)=\rho_{x}\left(\gamma_{2}\right)^{-1} \cdot\left(\gamma_{1} \star x\right)=$ $\rho_{x}\left(\gamma_{2}\right)^{-1} \rho_{x}\left(\gamma_{1}\right)^{-1} \cdot x$. Since $x \in M^{\text {free }}$, we have $\rho_{x}\left(\gamma_{1} \gamma_{2}\right)=\rho_{x}\left(\gamma_{1}\right) \rho_{x}\left(\gamma_{2}\right)$.

Lemma 5.10. For any $x^{\prime} \in M^{\text {free }}$, if $\left[x^{\prime}\right]_{G}=[x]_{G}$, then $\rho_{x^{\prime}}$ is conjugate with $\rho_{x}$.

Proof. There exists $g \in G$ such that $x^{\prime}=g x$. Then we have

$$
\rho_{x^{\prime}}(\gamma)^{-1} x^{\prime}=\gamma \star(g x)=g(\gamma \star x)=g \rho_{x}(\gamma)^{-1} x=g \rho_{x}(\gamma)^{-1} g^{-1} x^{\prime} .
$$

By the freeness, we have $\rho_{x^{\prime}}=g \rho_{x} g^{-1}$. 
For a fixed homomorphism $\rho: \Gamma \rightarrow G$, put $\left(M /{ }_{0} G\right)_{\rho}^{\Gamma}:=\left\{[x]_{G} \mid \rho_{x}\right.$ is conjugate with $\left.\rho\right\}$.

On the other hand, we can define a new $\Gamma$-action on $M$ by $x \mapsto \rho(\gamma) \gamma x$ for a given homomorphism $\rho: \Gamma \rightarrow G$. Needless to say when $x \in M$ is fixed by the $(\Gamma, \rho)$-action, $x$ satisfies $\gamma \star x=\rho(\gamma)^{-1} \cdot x$. We write these fixed points as $M^{\Gamma, \rho}$. Put $G^{\Gamma, \rho}:=\{g \in G \mid$ $\rho(\gamma) g \rho(\gamma)^{-1}=g$ for all $\left.\gamma \in \Gamma\right\}$.

Proposition 5.11. $\left(M / /{ }_{0} G\right)_{\rho}^{\Gamma}$ is isomorphic to $M^{\Gamma, \rho} / /{ }_{0} G^{\Gamma, \rho}$.

Proof. Since $x \in M^{\Gamma, \rho}$ satisfies $\gamma x=\rho(\gamma)^{-1} x$, we can regard that $\rho$ is $\rho_{x}$ and $x$ represents a point in $\left(M / /{ }_{0} G\right)_{\rho}^{\Gamma}$. Thus we have a map $M^{\Gamma, \rho} \rightarrow\left(M / /{ }_{0} G\right)_{\rho}^{\Gamma}$.

First we claim this map is surjective from $\left(M^{\text {free }}\right)^{\Gamma, \rho}$ to $\left(M^{\text {free }} / /_{0} G\right)_{\rho}^{\Gamma}$. Take an element $x \in M^{\text {free }}$ which represents a point in $\left(M^{\text {free }} / /{ }_{0} G\right)_{\rho}^{\Gamma}$. Since $\rho_{x}$ is conjugate with $\rho$, there exists $g \in G$ which satisfies $\rho_{x}=g \rho g^{-1}$. Put $x^{\prime}:=g^{-1} x$, we have

$$
\gamma x^{\prime}=g^{-1} \gamma x=g^{-1} \rho_{x}(\gamma)^{-1} x=\rho(\gamma)^{-1} g^{-1} x=\rho(\gamma)^{-1} x^{\prime} .
$$

Thus $x^{\prime}$ enters in $\left(M^{\text {free }}\right)^{\Gamma, \rho}$ and it means the surjectivity.

Suppose $x, x^{\prime} \in\left(M^{\text {free }}\right)^{\Gamma, \rho}$ and there exists $g \in G$ such that $x=g x^{\prime}$. Then we have

$$
x=\rho(\gamma) \gamma x=\rho(\gamma) \gamma g x^{\prime}=\rho(\gamma) g \rho(\gamma)^{-1} \rho(\gamma) \gamma x^{\prime}=\rho(\gamma) g \rho(\gamma)^{-1} x^{\prime},
$$

so we get $g=\rho(\gamma) g \rho(\gamma)^{-1}$ by the freeness. This induces an isomorphism $\left(M^{\text {free }}\right)^{\Gamma, \rho}$ $/ /{ }_{0} G^{\Gamma, \rho} \rightarrow\left(M^{\text {free }} / /{ }_{0} G\right)_{\rho}^{\Gamma}$.

For $x \in M \backslash M^{\text {free }}$, it is enough to consider a sequence $\left\{x_{n}\right\} \subset M^{\text {free }}$ such that $x_{n}$ converges to $x$ as $n \rightarrow \infty$. Therefore $\left(M / /{ }_{0} G\right)_{\rho}^{\Gamma}$ and $M^{\Gamma, \rho} / /{ }_{0} G^{\Gamma, \rho}$ are isomorphic.

From now we apply Proposition 5.11 to $\left(\mu_{\mathbb{C}}^{-1}(0) / /{ }_{0} G_{\mathbb{C}}\right)^{\Gamma}$. Take $[x]_{G_{\mathbb{C}}} \in\left(\mu_{\mathbb{C}}^{-1}(0) / /{ }_{0} G_{\mathbb{C}}\right)^{\Gamma}$ $\cong S^{m}(\mathrm{TN})^{\Gamma}$. By Remark 4.21 and Proposition 5.8, $x$ is represented as

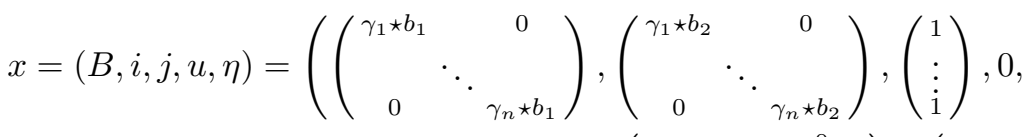

$$
\begin{aligned}
& \left.\left(\begin{array}{ccc}
\gamma_{1} \star u_{A} & & 0 \\
& \ddots & \\
0 & & \gamma_{n} \star u_{A}
\end{array}\right),\left(\begin{array}{ccc}
\gamma_{1} \star \eta_{A} & & 0 \\
& \ddots & \\
0 & & \gamma_{n} \star \eta_{A}
\end{array}\right)\right) \text {, }
\end{aligned}
$$

where $\left\{\gamma_{1}, \cdots, \gamma_{n}\right\}=\Gamma$ and we write $\gamma_{k} \star\left(b_{1}, b_{2}, u, \eta\right)$ as $\left(\gamma_{k} \star b_{1}, \gamma_{k} \star b_{2}, \gamma_{k} \star u, \gamma_{k} \star \eta\right)$. Here the $\Gamma$-action is defined on such $\{x\}$, and in fact we can extend the $\Gamma$-action on $\mathbb{M}_{\mathbb{C}}$ in the case appearing later.

$G_{\mathbb{C}}$ acts freely on above $\{x\}$, so $\rho(\gamma)=\left(\rho_{-}(\gamma)^{-1}, \rho_{0}(\gamma)^{-1}, \rho_{+}(\gamma)^{-1}\right) \in G_{\mathbb{C}}$ is uniquely determined. In order to know $\rho(\gamma)$ explicitly, we have to solve the following equations:

$$
\begin{aligned}
\gamma \star B_{-+} & =\rho_{-}(\gamma)^{-1} B_{-+} \rho_{+}(\gamma), & \gamma \star B_{+-} & =\rho_{+}(\gamma)^{-1} B_{+-} \rho_{-}(\gamma), \\
\gamma \star i & =\rho_{0}(\gamma)^{-1} i, & \gamma \star j & =j \rho_{0}(\gamma), \\
\gamma \star u_{L} & =\rho_{+}(\gamma)^{-1} u_{L} \rho_{-}(\gamma), & \gamma \star u_{R} & =\rho_{+}(\gamma)^{-1} u_{R} \rho_{0}(\gamma), \\
\gamma \star \eta_{L} & =\rho_{-}(\gamma)^{-1} \eta_{L} \rho_{-}(\gamma), & \gamma \star \eta_{R} & =\rho_{0}(\gamma)^{-1} \eta_{R} \rho_{0}(\gamma) .
\end{aligned}
$$

The following subsection we will solve them particularly in the case of $A_{n}$ and $D_{n}$. Note that there are two reasons why we chose $i$ as ${ }^{t}(1, \cdots, 1)$. The first is that $G_{\mathbb{C}}$ acts freely for such $i$, as we mentioned in Remark 4.21. The second is that the solution $\rho$ of the equations (5.12) enters $G$ not only $G_{\mathbb{C}}$. This is cleared up by the later calculation.

Therefore by Proposition 5.11, we get $\mathrm{TN} / \Gamma \cong\left(\mu_{\mathbb{C}}^{-1}(0) /{ }_{0} G_{\mathbb{C}}\right)^{\Gamma} \cong\left(\mu_{\mathbb{C}}^{-1}(0)\right)^{\Gamma} / /_{0} G_{\mathbb{C}}^{\Gamma}$ 
as algebraic varieties. And we consider the way to get a similar isomorphism as hyperKähler manifolds, that is, an isomorphism between $\left(\mu_{\mathbb{R}}^{-1}(0) \cap \mu_{\mathbb{C}}^{-1}(0) / G\right)^{\Gamma}$ and $\left(\mu_{\mathbb{R}}^{-1}(0) \cap\right.$ $\left.\mu_{\mathbb{C}}^{-1}(0)\right)^{\Gamma} / G^{\Gamma}$. Furthermore, we consider how to construct $\left(\mu_{\mathbb{R}}^{-1}(0) \cap \mu_{\mathbb{C}}^{-1}(0)\right)^{\Gamma} / G^{\Gamma}$ as a hyper-Kähler quotient.

First we recall that there exists the biholomorphism $\left(\mu_{\mathbb{R}}^{-1}(0) \cap \mu_{\mathbb{C}}^{-1}(0)\right) / G \cong \mu_{\mathbb{C}}^{-1}(0)$ $/ /{ }_{0} G_{\mathbb{C}}$ by Proposition 4.7. By this biholomorphism we can take a representative $[x]_{G} \in$ $\left.\left(\mu_{\mathbb{R}}^{-1}(0) \cap \mu_{\mathbb{C}}^{-1}(0)\right) / G\right)^{\Gamma}$ as

$$
x=\left(\left(\begin{array}{ccc}
\gamma_{1} \star b_{1} & & 0 \\
& \ddots & \\
0 & & \gamma_{n} \star b_{1}
\end{array}\right),\left(\begin{array}{ccc}
\gamma_{1} \star b_{2} & & 0 \\
& \ddots & \\
0 & & \gamma_{n} \star b_{2}
\end{array}\right), 0,0,\left(\begin{array}{ccc}
\gamma_{1} \star u & & 0 \\
& \ddots & \\
0 & & \gamma_{n} \star u
\end{array}\right),\left(\begin{array}{ccc}
\gamma_{1} \star \eta & & 0 \\
& \ddots & \\
0 & & \gamma_{n} \star \eta
\end{array}\right)\right) \text {, }
$$

where $\left[\left(b_{1}, b_{2}, u, \eta\right)\right]_{S^{1}} \in \mathrm{TN}$. Since the above solution $\rho$ is in $G$, we can define the same $\Gamma$-action on $\mathbb{M}_{\mathbb{C}}$ as before. It is clear that $\left\{x \in \mu^{-1}(0) \mid \gamma x=\rho(\gamma)^{-1} x\right\}=\left\{x \in \mathbb{M}_{\mathbb{C}}^{\Gamma} \mid\right.$ $\mu(x)=0\}$ holds.

Then we get a map from $\mu_{\mathbb{R}}^{-1}(0) \cap \mu_{\mathbb{C}}^{-1}(0) \cap \mathbb{M}_{\mathbb{C}}^{\Gamma}$ to $\left(\mu_{\mathbb{R}}^{-1}(0) \cap \mu_{\mathbb{C}}^{-1}(0) / G\right)^{\Gamma}$ like Proposition 5.11. However we cannot make the same argument to show the injiectivity of the map $\mu^{-1}(0) \cap \mathbb{M}_{\mathbb{C}}^{\Gamma} / G^{\Gamma} \rightarrow\left(\mu^{-1}(0) / G\right)^{\Gamma}$ as Proposition 5.11 because there is no point in $\mu^{-1}(0)$ on which $G$ acts freely. To clear up this problem, it is enough to check $\operatorname{Stab}(x) \cap G^{\Gamma}$ acts trivially on $\mu^{-1}(0) \cap \mathbb{M}_{\mathbb{C}}^{\Gamma}$ for any $x \in \mu^{-1}(0)$.

Last we check $\mu_{\mathbb{R}}^{-1}(0) \cap \mu_{\mathbb{C}}^{-1}(0) \cap \mathbb{M}_{\mathbb{C}}^{\Gamma} / G^{\Gamma}$ is obtained by a hyper-Kähler quotient of $\mathbb{M}_{\mathbb{C}}^{\Gamma}$ by $G^{\Gamma}$. Let $\mu^{\Gamma}$ be the moment map defined by the $G^{\Gamma}$-action. Since $G^{\Gamma}$ is a (closed) subgroup of $G$, the moment map $\mu^{\Gamma}$ is given by the orthogonal projection of $\mu$ on $\left(\operatorname{Lie} G^{\Gamma}\right)^{*}$. Then it is enough to check that the image of the restriction map $\left.\mu\right|_{\mathbb{M}_{C}^{\Gamma}}$ is included in $\left(\operatorname{Lie} G^{\Gamma}\right)^{*}$.

Therefore we can get an isomorphism

$$
\mathrm{TN} / \Gamma \cong\left(\mu_{\mathbb{R}}^{-1}(0) \cap \mu_{\mathbb{C}}^{-1}(0) / G\right)^{\Gamma} \cong \mu_{\mathbb{R}}^{\Gamma^{-1}}(0) \cap \mu_{\mathbb{C}}^{\Gamma^{-1}}(0) / G^{\Gamma},
$$

as hyper-Kähler manifolds, if the following condition hold:

\section{CONDITION 5.13}

(1) If $\rho(\gamma) \in G_{\mathbb{C}}$ is the solution of (5.12), it is in $G$.

(2) $\operatorname{Stab}(x) \cap G^{\Gamma}$ acts trivially on $\mu^{-1}(0) \cap \mathbb{M}_{\mathbb{C}}^{\Gamma}$ for any $x \in \mu^{-1}(0)$.

(3) The image of the restriction map $\left.\mu\right|_{\mathbb{M}_{C}^{\Gamma}}$ is included in $\left(\operatorname{Lie} G^{\Gamma}\right)^{*}$.

In the following subsections, we calculate $\mathbb{M}_{\mathbb{C}}^{\Gamma}$ and $G^{\Gamma}$ for $\Gamma=A_{n}, D_{n}$ cases.

EXAMPLE 5.14. In Kronheimer's situation [Kr2], one can check that the homomorphism $\rho$ and $M_{\mathbb{C}}^{\Gamma}$ coincide with the regular representation of the finite groups of $S U(2)$ and $(Q \otimes \operatorname{End}(R))^{\Gamma}$ respectively.

\section{3. $\Gamma=A_{n}$ case}

By using the results in $\S 5.2$, we get TN $/ A_{n}$ as a hyper-Kähler quotient. Because $A_{n}=$ $\left\langle\omega_{n+1}\right\rangle(n \geq 0)$, so we can take $(B, i, j, u, \eta)$ as

$$
\left(\left(\begin{array}{cccc}
b_{1} & 0 & \cdots & 0 \\
0 & \zeta b_{1} & \cdots & 0 \\
\vdots & \vdots & \ddots & \vdots \\
0 & 0 & \cdots & \zeta^{n} b_{1}
\end{array}\right),\left(\begin{array}{cccc}
b_{2} & 0 & \cdots & 0 \\
0 & \zeta^{-1} b_{2} & \cdots & 0 \\
\vdots & \vdots & \ddots & \vdots \\
0 & 0 & \cdots & \zeta^{-n} b_{2}
\end{array}\right),\left(\begin{array}{c}
1 \\
\vdots \\
\vdots \\
1
\end{array}\right), 0,\left(\begin{array}{cccc}
u_{A} & 0 & \cdots & 0 \\
0 & u_{A} & \cdots & 0 \\
\vdots & \vdots & \ddots & \vdots \\
0 & 0 & \cdots & u_{A}
\end{array}\right),\left(\begin{array}{cccc}
\eta_{A} & 0 & \cdots & 0 \\
0 & \eta_{A} & \cdots & 0 \\
\vdots & \vdots & \ddots & \vdots \\
0 & 0 & \cdots & \eta_{A}
\end{array}\right)\right),
$$


where $\zeta=\zeta_{n+1}$. And by definition of the $\Gamma$-action on Taub-NUT space, we have

$\omega \star(B, i, j, u, \eta)=$

$$
\left(\left(\begin{array}{cccc}
\zeta b_{1} & 0 & \cdots & 0 \\
0 & \zeta^{2} b_{1} & \cdots & 0 \\
\vdots & \vdots & \ddots & \vdots \\
0 & 0 & \cdots & b_{1}
\end{array}\right),\left(\begin{array}{cccc}
\zeta^{-1} b_{2} & 0 & \cdots & 0 \\
0 & \zeta^{-2} b_{2} & \cdots & 0 \\
\vdots & \vdots & \ddots & \vdots \\
0 & 0 & \cdots & b_{2}
\end{array}\right),\left(\begin{array}{c}
1 \\
\vdots \\
\vdots \\
1
\end{array}\right), 0,\left(\begin{array}{cccc}
u_{A} & 0 & \cdots & 0 \\
0 & u_{A} & \cdots & 0 \\
\vdots & \vdots & \ddots & \vdots \\
0 & 0 & \cdots & u_{A}
\end{array}\right),\left(\begin{array}{cccc}
\eta_{A} & 0 & \cdots & 0 \\
0 & \eta_{A} & \cdots & 0 \\
\vdots & \vdots & \ddots & \vdots \\
0 & 0 & \cdots & \eta_{A}
\end{array}\right)\right) .
$$

This action is extended on $\mathbb{M}_{\mathbb{C}}$ as $\omega \star(B, i, j, u, \eta)=\left(\zeta B_{-+}, \zeta^{-1} B_{+-}, i, j, u, \eta\right)$. And the

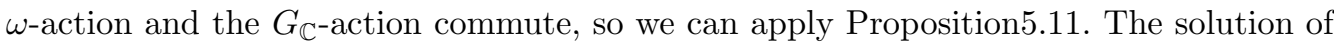
(5.12) is given by

$$
\rho_{-}(\omega)^{-1}=\rho_{0}(\omega)^{-1}=\rho_{+}(\omega)^{-1}=\left(\begin{array}{cccccc}
0 & 1 & 0 & 0 & \cdots & 0 \\
0 & 0 & 1 & 0 & \cdots & 0 \\
0 & 0 & 0 & 1 & 0 \\
0 & 0 & \vdots & \ddots & \ddots & 0 \\
0 & 0 & 0 & 0 & 0 \\
1 & 0 & 0 & 0 & \cdots & 0 \\
0
\end{array}\right) .
$$

In this case each $\rho_{\bullet}$ is a homomorphism from $A_{n}$ to $U(n+1)$. By a change of basis, $\rho$ can be described as

$$
\rho_{-}(\omega)=\rho_{0}(\omega)=\rho_{+}(\omega)=\operatorname{diag}\left(1, \zeta, \zeta^{2}, \cdots, \zeta^{n}\right) .
$$

This means that each $\rho_{\bullet}$ is actually the regular representation of $A_{n}$. For this $\rho$, we apply Proposition5.11. A point in $\mathbb{M}_{\mathbb{C}}^{\Gamma, \rho}=\mathbb{M}_{\mathbb{C}}^{\Gamma}$ is represented as

$$
\begin{aligned}
& \left(\left(\begin{array}{cccccc}
0 & b_{01} & 0 & 0 & \cdots & 0 \\
0 & 0 & b_{12} & 0 & \cdots & 0 \\
0 & 0 & 0 & b_{23} & & 0 \\
\vdots & \vdots & \vdots & & \ddots & \\
0 & 0 & 0 & 0 & \ddots & b_{n-1, n} \\
b_{n 0} & 0 & 0 & 0 & \cdots & 0
\end{array}\right),\left(\begin{array}{cccccc}
0 & 0 & 0 & \cdots & 0 & b_{0 n} \\
b_{10} & 0 & 0 & \cdots & 0 & 0 \\
0 & b_{21} & 0 & \cdots & 0 & 0 \\
0 & 0 & b_{32} & & 0 & 0 \\
\vdots & \vdots & & \ddots & & \\
0 & 0 & 0 & & b_{n, n-1} & 0
\end{array}\right),\right.
\end{aligned}
$$

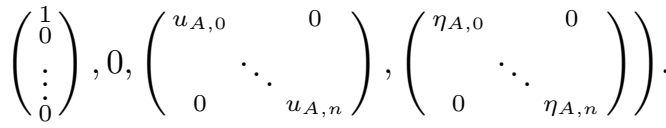

It satisfies $\mu_{\mathbb{C}}=0$ if and only if

$$
\left\{\begin{array}{l}
b_{k, k+1} b_{k+1, k}+2 \eta_{L, k}=0 \\
-2 u_{L, k} \eta_{L, k} u_{L, k}^{-1}+2 u_{R, k} \eta_{R, k} u_{R, k}^{-1}=0 \\
-2 \eta_{R, k}-b_{k, k-1} b_{k-1, k}=0
\end{array}\right.
$$

And $G^{\Gamma} \subset G$ is given by

$$
G^{\Gamma}=\left\{\left(\operatorname{diag}\left(g_{-, 0}, \cdots, g_{-, n}\right), \operatorname{diag}\left(g_{0,0}, \cdots, g_{0, n}\right), \operatorname{diag}\left(g_{+, 0}, \cdots, g_{+, n}\right)\right) \in G\right\} .
$$

These data are described as the following diagram:

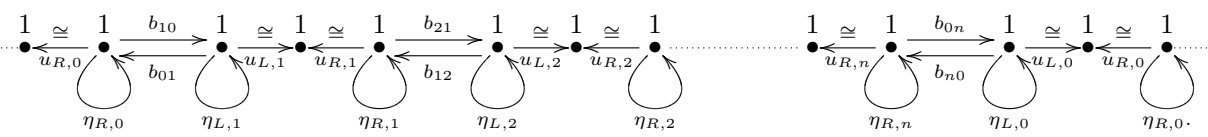

Notice that the both ends of the above diagram are connected. And by Kronheimer's theorem, each $\{(u, \eta)\}$ is regarded as the moduli space of the solutions of 1-dimensional Nahm's equation, so we can get a new bow by replacing

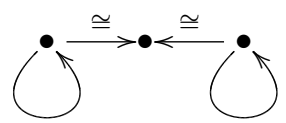

with 


\section{YUUYA TAKAYAMA}

It is clear that Condition 5.13 holds in this case, so $\mathrm{TN} / A_{n}$ is constructed by a hyperKähler quotient corresponding to the above diagram. In $\S 6$, we study such a hyper-Kähler quotient.

5.4. $\Gamma=D_{n}$ case

By using the results in $\S 5.2$, we get $\mathrm{TN} / D_{n}$ as a hyper-Kähler quotient. Because $D_{n}=$ $\left\langle\omega_{m}, \iota\right\rangle(m=2 n-4, n \geq 3)$, and we order them as $\left\{1, \omega, \omega^{2}, \cdots, \omega^{m-1}, \iota, \iota \omega, \iota \omega^{2}, \cdots\right.$, $\left.\iota \omega^{m-1}\right\}$, where $\omega$ denotes $\omega_{m}$. Then we can take $(B, i, j, u, \eta)$ as

$$
\begin{aligned}
B_{-+} & =\operatorname{diag}\left(b_{1}, \zeta b_{1}, \cdots, \zeta^{m-1} b_{1},-b_{2},-\zeta^{-1} b_{2}, \cdots,-\zeta^{-(m-1)} b_{2}\right), \\
B_{+-} & =\operatorname{diag}\left(b_{2}, \zeta^{-1} b_{2}, \cdots, \zeta^{-(m-1)} b_{2}, b_{1}, \zeta b_{1}, \cdots, \zeta^{m-1} b_{1}\right), \\
i & =\operatorname{diag}(1,1, \cdots, 1,1,1, \cdots, 1), \quad j=0, \\
u_{L} & =\operatorname{diag}\left(u_{L}, u_{L}, \cdots, u_{L}, u_{R}, u_{R}, \cdots, u_{R}\right), \\
u_{R} & =\operatorname{diag}\left(u_{R}, u_{R}, \cdots, u_{R}, u_{L}, u_{L}, \cdots, u_{L}\right), \\
\eta_{L} & =\operatorname{diag}\left(\eta_{L}, \eta_{L}, \cdots, \eta_{L},-\eta_{R},-\eta_{R}, \cdots,-\eta_{R}\right), \\
\eta_{R} & =\operatorname{diag}\left(\eta_{R}, \eta_{R}, \cdots, \eta_{R},-\eta_{L},-\eta_{L}, \cdots,-\eta_{L}\right) .
\end{aligned}
$$

And the $\Gamma$-action can be extended on $\mathbb{M}_{\mathbb{C}}$ as

$$
\begin{aligned}
\omega \star(B, i, j, u, \eta) & =\left(\zeta B_{-+}, \zeta^{-1} B_{+-}, i, j, u_{L}, u_{R}, \eta_{L}, \eta_{R}\right), \\
\iota \star(B, i, j, u, \eta) & =\left(B_{+-},-B_{-+}, i, j, u_{R}, u_{L},-\eta_{R},-\eta_{L}\right) .
\end{aligned}
$$

But the $\iota$-action does not commute with the $G_{\mathbb{C}}$-action. In order to decompose the elemental bow, we modify Proposition5.11. For $g=\left(g_{-}, g_{0}, g_{+}\right) \in G_{\mathbb{C}}$, put $g^{\iota}:=\left(g_{+}, g_{0}, g_{-}\right)$. Then we have

$$
\iota \star(g \cdot(B, i, j, u, \eta))=g^{\iota} \cdot(\iota \star(B, i, j, u, \eta)) .
$$

Thus rewriting $g$ by $g^{\iota}$ if necessary, we have the same result as Proposition5.11.

Hence the solution of (5.12) is given by

$$
\begin{gathered}
\rho_{-}(\omega)^{-1}=\rho_{0}(\omega)^{-1}=\rho_{+}(\omega)^{-1}=\left(\begin{array}{cc}
P_{m} & 0 \\
0 & { }^{t} P_{m}
\end{array}\right), \\
\rho_{-}(\iota)^{-1}=\rho_{0}(\iota)^{-1}=\rho_{+}(\iota)^{-1}=\left(\begin{array}{cc}
0 & \operatorname{id}_{m} \\
Q_{m} & 0
\end{array}\right),
\end{gathered}
$$

where

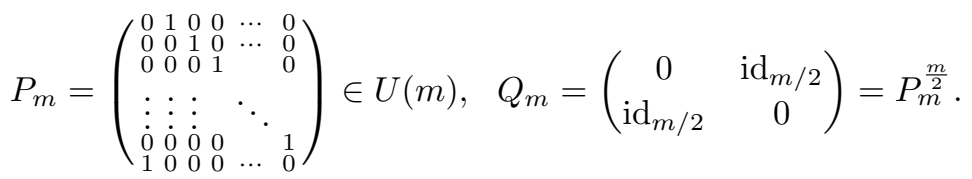

Here $\rho_{0}$ is a homomorphism from $D_{n}$ to $U(2 m)$. Each $\rho_{ \pm}$is not a homomorphism but

$$
\omega \mapsto\left(\begin{array}{cc}
\rho_{-}(\omega) & 0 \\
0 & \rho_{+}(\omega)
\end{array}\right), \quad \iota \mapsto\left(\begin{array}{cc}
0 & \rho_{-}(\iota) \\
\rho_{+}(\iota) & 0
\end{array}\right)
$$

is a homomorphism from $D_{n}$ to $U(4 m)$. By using this representation, a change of basis is written as

$$
\begin{aligned}
\rho_{0}(\omega) & \mapsto g_{0} \rho_{0}(\omega) g_{0}^{-1}, & \left(\begin{array}{cc}
\rho_{-}(\omega) & 0 \\
0 & \rho_{+}(\omega)
\end{array}\right) & \mapsto\left(\begin{array}{cc}
g_{-} & 0 \\
0 & g_{+}
\end{array}\right)\left(\begin{array}{cc}
\rho_{-}(\omega) & 0 \\
0 & \rho_{+}(\omega)
\end{array}\right)\left(\begin{array}{cc}
g_{-} & 0 \\
0 & g_{+}
\end{array}\right)^{-1}, \\
\rho_{0}(\iota) & \mapsto g_{0} \rho_{0}(\iota) g_{0}^{-1}, & \left(\begin{array}{cc}
0 & \rho_{-}(\iota) \\
\rho_{+}(\iota) & 0
\end{array}\right) & \mapsto\left(\begin{array}{cc}
g_{-} & 0 \\
0 & g_{+}
\end{array}\right)\left(\begin{array}{cc}
0 & \rho_{-}(\iota) \\
\rho_{+}(\iota) & 0
\end{array}\right)\left(\begin{array}{cc}
g_{-} & 0 \\
0 & g_{+}
\end{array}\right)^{-1} .
\end{aligned}
$$


Then $\rho_{\bullet}$ can be described as

$$
\begin{aligned}
& \rho_{-}(\omega)=\rho_{0}(\omega)=\rho_{+}(\omega)=\operatorname{diag}\left(\mathrm{id}_{2}, \zeta^{-1} \mathrm{id}_{2}, \zeta \mathrm{id}_{2}, \cdots, \zeta^{-m} \operatorname{id}_{2}, \zeta^{m} \operatorname{id}_{2},-\mathrm{id}_{2}\right)
\end{aligned}
$$

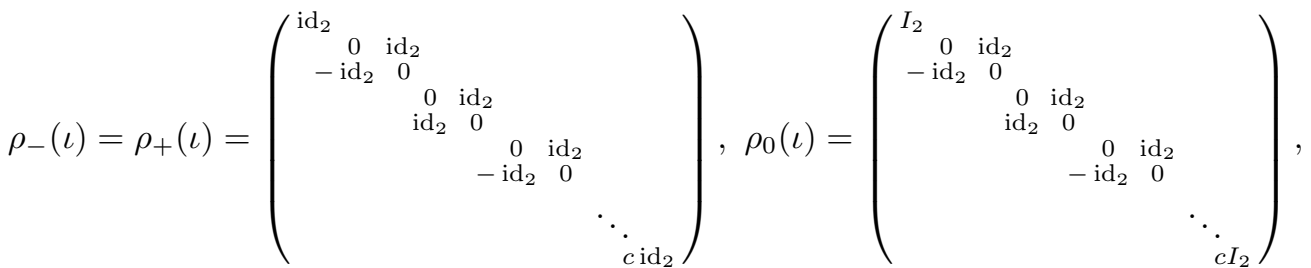

where $c=\sqrt{-1}$ (resp. 1) when $n$ is odd (resp. even) and $I_{2}=\operatorname{diag}(1,-1)$. This means that $\rho_{0}$ is the regular representation of $D_{n}$. A point in $\mathbb{M}^{\Gamma}$ is represented as

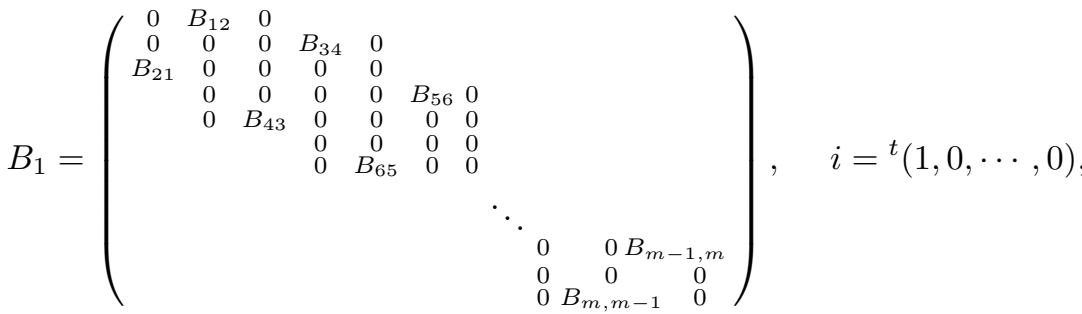

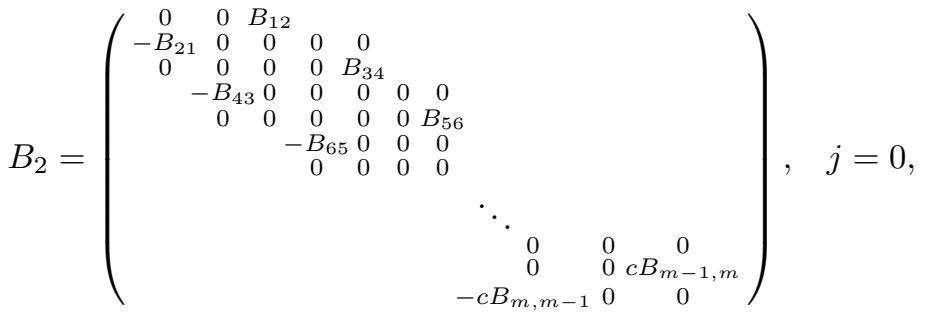

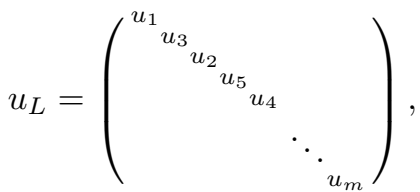

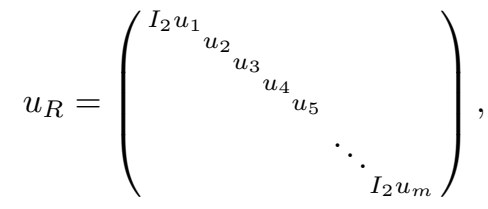

$\eta_{L}=\left(\begin{array}{ccccc}\eta_{1} & & & & \\ { }^{\eta_{3}} & \eta_{2} & & & \\ & \eta_{2} & \eta_{5} & & \\ & & \eta_{4} & & \\ & & & \ddots & \\ & & & \eta_{m}\end{array}\right)$,

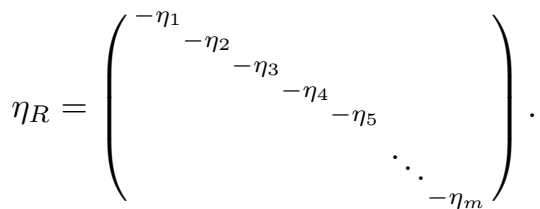

It satisfies $\mu_{\mathbb{C}}=0$ if and only if

$$
\left\{\begin{array}{l}
-B_{k, k+1} B_{k+1, k}+2 u_{k}=0 \\
-2 u_{1} \eta_{1} u_{1}^{-1}-2 I_{2} u_{1} \eta_{1} u_{1}^{-1} I_{2}=0 \\
-2 u_{k} \eta_{k} u_{k}^{-1}-2 u_{k-1} \eta_{k-1} u_{k-1}^{-1}=0 \\
2 \eta_{k}-B_{k, k+1} B_{k+1, k}=0 \\
B_{k+1, k} B_{k, k+1}-2 \eta_{k+1}=0 \\
2 u_{m} \eta_{m} u_{m}^{-1}+2 I_{2} u_{m} \eta_{m} u_{m}^{-1} I_{2}=0 .
\end{array}\right.
$$


And $G^{\Gamma}=\left\{\left(g_{-}, g_{0}, g_{+}\right)\right\} \subset G$ is given by

$$
\begin{aligned}
g_{-} & =\operatorname{diag}\left(g_{-, 1}, g_{-, 3}, g_{-, 2}, g_{-, 5}, g_{-, 4}, \cdots, g_{-, m}\right), \\
g_{0} & =\operatorname{diag}\left(g_{0,1}, g_{0,2}, g_{0,3}, g_{0,4}, g_{0,5}, \cdots, g_{0, m}\right), \\
g_{+} & =\operatorname{diag}\left(g_{+, 1}, g_{+, 2}, g_{+, 3}, g_{+, 4}, g_{+, 5}, \cdots, g_{+, m}\right),
\end{aligned}
$$

where $g_{\bullet}, \bullet \in U(2)$ and $g_{0,1}, g_{0, m}$ satisfy $g_{0, \bullet}=I_{2} g_{0, \bullet} I_{2}$. Here for $A=\left(\begin{array}{ll}a & b \\ c & d\end{array}\right)$, we have $\left(\begin{array}{cc}1 & 0 \\ 0 & -1\end{array}\right)\left(\begin{array}{ll}a & b \\ c & d\end{array}\right)\left(\begin{array}{cc}1 & 0 \\ 0 & -1\end{array}\right)=\left(\begin{array}{cc}a & -b \\ -c & d\end{array}\right)$, so $A+I_{2} A I_{2}=0$ means diagonal entries are 0 and $A=I_{2} A I_{2}$ means off diagonal entries are 0 . Especially $g_{0, \bullet}=\left(\begin{array}{cc}g_{0}^{1,1}, & 0 \\ 0 & g_{0, \boldsymbol{\bullet}}^{2,2}\end{array}\right) \in U(1) \times$ $U(1),(\bullet=1, m)$. Then we get a new diagram:

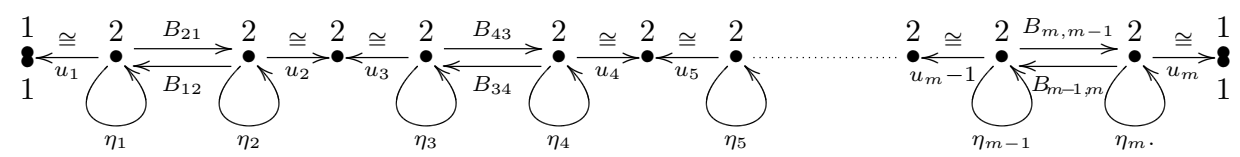

Here means that the acting group on it is $U(1) \times U(1) \subset U(2)$ (diagonal subgroup).

In the same way as the previous subsection, we can get a new bow by Kronheimer's theorem. And it is also clear that Condition 5.13 holds in this case, so $\mathrm{TN} / D_{n}$ is constructed by a hyper-Kähler quotient corresponding to the above diagram. In $\S 7$, we study such a hyper-Kähler quotient.

\section{6. $A_{n}$-type bow varieties}

In this section, we study the properties of $A_{n}$-type bow varieties (see also [C2, $\left.§ 4.2\right]$ ).

6.1. An-type bow

We consider the following diagram:

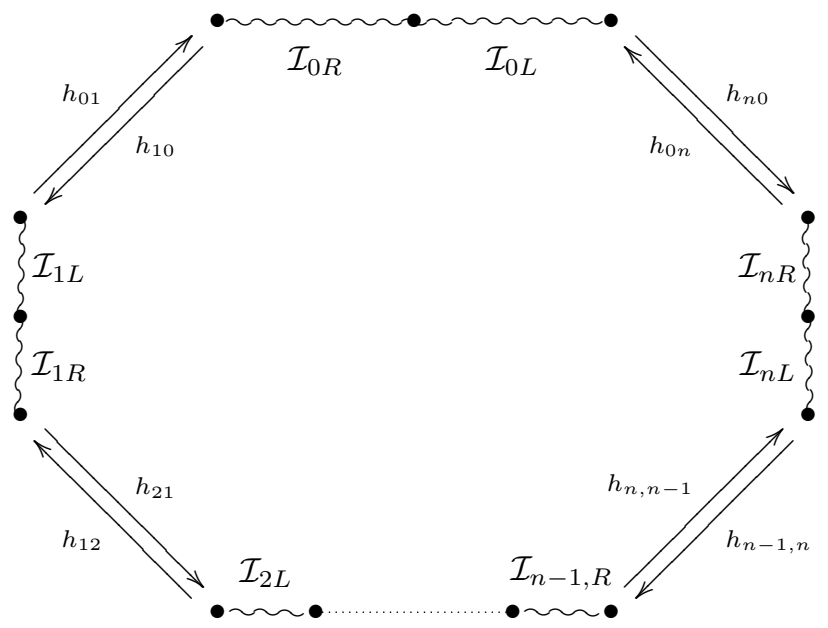

where $\mathcal{I}_{\sigma L}=\left\{-l_{\sigma} \leq s_{\sigma} \leq 0\right\}$ and $\mathcal{I}_{\sigma R}=\left\{0 \leq s_{\sigma} \leq l_{\sigma}\right\}$. For $\vec{l}=\left(l_{0}, \cdots, l_{n}\right)$, we assume $|\vec{l}|=\sum l_{\sigma}>0$. Put $\Omega=\left\{h_{\sigma+1, \sigma}\right\}$ and $\bar{\Omega}=\left\{h_{\sigma, \sigma+1}\right\}$.

We give rank-1 trivial hermitian vector bundles $\pi_{\sigma}: V_{\sigma} \rightarrow \mathcal{I}_{\sigma L} \cup \mathcal{I}_{\sigma R}$. We describe $\pi_{\sigma}^{-1}\left(s_{\sigma}\right)$ as $V_{\sigma}\left(s_{\sigma}\right)$. In the same way as $\S 4.1$., let us define complex vector spaces $\mathbb{M}_{\Omega}^{A_{n}}$, 
$\mathbb{M}_{\bar{\Omega}}^{A_{n}}$ and $\mathbb{M}^{A_{n}}$ respectively by

$$
\begin{aligned}
\mathbb{M}_{\Omega}^{A_{n}} & :=\bigoplus_{h \in \Omega} \operatorname{Hom}\left(V(o(h)), V(i(h)) \cong \bigoplus_{\sigma}\left\{b_{\sigma+1, \sigma} \in \mathbb{C}\right\},\right. \\
\mathbb{M}_{\bar{\Omega}}^{A_{n}} & :=\bigoplus_{h \in \bar{\Omega}} \operatorname{Hom}\left(V(o(h)), V(i(h)) \cong \bigoplus_{\sigma}\left\{b_{\sigma, \sigma+1} \in \mathbb{C}\right\},\right. \\
\mathbb{M}^{A_{n}}(\vec{l}) & :=\mathbb{M}_{\Omega}^{A_{n}} \oplus \mathbb{M}_{\bar{\Omega}}^{A_{n}} \oplus \bigoplus_{\sigma=0}^{n} \mathcal{H}_{\sigma L} \oplus \bigoplus_{\sigma=0}^{n} \mathcal{H}_{\sigma R} .
\end{aligned}
$$

When $l_{\sigma}=0$ for some $\sigma$, we replace the vector bundle $\pi_{\sigma}: V_{\sigma} \rightarrow \mathcal{I}_{\sigma L} \cup \mathcal{I}_{\sigma R}$ with a 1-dimensional vector space $V_{\sigma}^{\prime}$ and remove $\mathcal{H}_{\sigma L} \oplus \mathcal{H}_{\sigma R}$ from $\mathbb{M}^{A_{n}}(\vec{l})$. This corresponds to taking the limit $l_{\sigma} \rightarrow 0$ as (4.14).

A group $\mathcal{G}^{A_{n}}=\prod_{\sigma} \mathcal{G}_{\sigma}=\prod\left\{g_{\sigma}=\left(g_{\sigma L}, g_{\sigma R}\right) \in L_{2}^{2}\left(\mathcal{I}_{\sigma L}, U(1)\right) \times L_{2}^{2}\left(\mathcal{I}_{\sigma R}, U(1)\right)\right.$ $\left.g_{\sigma L}(0)=g_{\sigma R}(0)\right\}$ acts on $\mathbb{M}^{A_{n}}(\vec{l})$ as $\S 4$. We have a hyper-Kähler moment map $\mu$ and the explicit form is as follows:

$$
\begin{aligned}
& \mu_{\mathbb{R}}= \begin{cases}\frac{-\sqrt{-1}}{2}\left(b_{\sigma, \sigma-1} b_{\sigma, \sigma-1}^{*}-b_{\sigma-1, \sigma}^{*} b_{\sigma-1, \sigma}\right)+T_{1}^{\sigma L}\left(-l_{\sigma}\right) & \text { at } s_{\sigma}=-l_{\sigma} \\
\frac{d}{d s} T_{1}^{\sigma A} & \text { at } s_{\sigma} \in\left(-l_{\sigma}, 0\right),\left(0, l_{\sigma}\right) \\
-T_{1}^{\sigma L}(0)+T_{1}^{\sigma R}(0) & \text { at } s_{\sigma}=0 \\
\frac{-\sqrt{-1}}{2}\left(b_{\sigma, \sigma+1} b_{\sigma, \sigma+1}^{*}-b_{\sigma+1, \sigma}^{*} b_{\sigma+1, \sigma}\right)-T_{1}^{\sigma R}\left(l_{\sigma}\right) & \text { at } s_{\sigma}=l_{\sigma},\end{cases} \\
& \mu_{\mathbb{C}}= \begin{cases}b_{\sigma, \sigma-1} b_{\sigma-1, \sigma}+\left(T_{2}^{\sigma L}+\sqrt{-1} T_{3}^{\sigma L}\right)\left(-l_{\sigma}\right) & \text { at } s_{\sigma}=-l_{\sigma} \\
\frac{d}{d s}\left(T_{2}^{\sigma A}+\sqrt{-1} T_{3}^{\sigma A}\right) & \text { at } s_{\sigma} \in\left(-l_{\sigma}, 0\right),\left(0, l_{\sigma}\right) \\
-\left(T_{2}^{\sigma L}+\sqrt{-1} T_{3}^{\sigma L}\right)(0)+\left(T_{2}^{\sigma R}+\sqrt{-1} T_{3}^{\sigma R}\right)(0) & \text { at } s_{\sigma}=0 \\
-b_{\sigma, \sigma+1} b_{\sigma+1, \sigma}-\left(T_{2}^{\sigma R}+\sqrt{-1} T_{3}^{\sigma R}\right)\left(l_{\sigma}\right) & \text { at } s_{\sigma}=l_{\sigma} .\end{cases}
\end{aligned}
$$

Put $Z^{A_{n}}=\left\{\zeta=\left(\zeta_{\sigma}\right) \in\left(\operatorname{Lie} U(1)^{n+1}\right)^{*} \otimes(\mathbb{R} \oplus \mathbb{C})\right\}$. Choose an element $\zeta=\left(\zeta_{\mathbb{R}}, \zeta_{\mathbb{C}}\right) \in Z^{A_{n}}$, and define a hyper-Kähler quotient $\mathcal{M}_{\zeta}^{A_{n}}(\vec{l})$ of $\mathbb{M}^{A_{n}}(\vec{l})$ by $\mathcal{G}^{A_{n}}$ as follows:

$$
\mathcal{M}_{\zeta}^{A_{n}}(\vec{l}):=\left\{(b, T) \in \mathbb{M}^{A_{n}}(\vec{l}) \mid \mu(b, T)=\zeta \delta_{s_{\sigma}=0}\right\} / \mathcal{G}^{A_{n}} .
$$

We call this hyper-Kähler manifold an $A_{n}$-type bow variety.

Remark 6.2. The subgroup $\left\{(c, \cdots, c) \in \mathcal{G}^{A_{n}} \mid c \in U(1)\right\}$ acts on $\mathbb{M}^{A_{n}}(\vec{l})$ trivially, so when $\sum_{\sigma} \zeta_{\sigma} \neq 0, \mathcal{M}_{\zeta}^{A_{n}}$ is empty. Afterward we only consider the case $\sum_{\sigma} \zeta_{\sigma}=0$.

By Proposition 3.13, we can rewrite these data as

$$
\begin{aligned}
\mathbb{M}_{\mathbb{C}}^{A_{n}}(\vec{l}) & =\bigoplus_{\sigma}\left\{\left(b_{\sigma, \sigma-1}, b_{\sigma-1, \sigma}, u_{\sigma L}, \eta_{\sigma L}, u_{\sigma R}, \eta_{\sigma R}\right) \in \mathbb{C} \times \mathbb{C} \times \mathbb{C}^{*} \times \mathbb{C} \times \mathbb{C}^{*} \times \mathbb{C}\right\}, \\
G^{A_{n}} & =\prod_{\sigma}\left\{\left(g_{-}^{\sigma}, g_{0}^{\sigma}, g_{+}^{\sigma}\right) \in U(1) \times U(1) \times U(1)\right\} .
\end{aligned}
$$

When all the $l_{\sigma}$ is equal and $\zeta=0$, the $A_{n}$-type bow variety $\mathcal{M}_{0}^{A_{n}}(\vec{l})$ coincides with $\mathrm{TN} / A_{n}$ (c.f. $\S 5.3$ ).

\subsection{General properties of $A_{n}$-type bow varieties}

For the $A_{n}$-type bow variety, we can make the same arguments in $\S 4.2$.

Proposition 6.3. The $A_{n}$-type bow variety $\mathcal{M}_{\zeta}^{A_{n}}$ is algebraic. 
Proof. It is enough to define

$$
\chi(g)^{-1}=\prod\left(g_{0}^{\sigma}\right)^{\zeta \sigma / 2 \sqrt{-1}},
$$

instead of (4.8), then actually we have

$$
\mu_{\chi}(b, u, \eta)= \begin{cases}\mu_{\mathbb{R}} & \text { at } s_{\sigma}=-l_{\sigma} \\ \mu_{\mathbb{R}}+\zeta_{\sigma} & \text { at } s_{\sigma}=0 \\ \mu_{\mathbb{R}} & \text { at } s_{\sigma}=l_{\sigma} .\end{cases}
$$

Proposition 6.4. As algebraic varieties, $\mathcal{M}_{\zeta}^{A_{n}}(\vec{l})$ is isomorphic to an $A_{n}$-type quiver variety constructed by Kronheimer $[\mathbf{K r 2}]$.

Corollary 6.5. If $\zeta=\left(\zeta_{\sigma}\right)$ satisfies the following condition, $\mathcal{M}_{\zeta}^{A_{n}}(\vec{l})$ is smooth. Moreover the projection $\mathcal{M}_{\zeta}^{A_{n}} \rightarrow \mathcal{M}_{0}^{A_{n}}$ induced by (1.7) is the resolution of singularities.

(*) There does not exist $(j, k)$ which satisfies $j<k$ and $\sum_{j \leq \sigma<k} \zeta_{\sigma}=0$.

Proof. This follows immediately from Proposition 6.4 and [Kr2]. We write down the condition in $\left[\mathbf{K r} \mathbf{2}\right.$, Proposition 2.8.] in the case of $A_{n}$, then we have the condition (*).

6.3. Metrics of $A_{n}$-type bow varieties

In order to study the metric of the $A_{n}$-type bow variety, we construct $\mathcal{M}_{\zeta}^{A_{n}}$ explicitly like $\S 4.3$ (see also $[\mathbf{C 2}, \S 4.3]$ ).

First we solve the equations at $s_{\sigma}=l_{\sigma}$ and $s_{\sigma+1}=-l_{\sigma+1}$ :

$$
\begin{aligned}
& \left\{\begin{array}{l}
-\frac{1}{2}\left(\left|b_{\sigma, \sigma+1}\right|^{2}-\left|b_{\sigma+1, \sigma}\right|^{2}\right)-t_{1}^{\sigma R}=0 \\
-b_{\sigma, \sigma+1} b_{\sigma+1, \sigma}-\left(\sqrt{-1} t_{2}^{\sigma R}-t_{3}^{\sigma R}\right)=0,
\end{array}\right. \\
& \left\{\begin{array}{l}
-\frac{1}{2}\left(\left|b_{\sigma+1, \sigma}\right|^{2}-\left|b_{\sigma, \sigma+1}\right|^{2}\right)+t_{1}^{\sigma+1 L}=0 \\
b_{\sigma+1, \sigma} b_{\sigma, \sigma+1}+\left(\sqrt{-1} t_{2}^{\sigma+1 L}-t_{3}^{\sigma+1 L}\right)=0 .
\end{array}\right.
\end{aligned}
$$

Put $\left(2 x_{\sigma 1},-\sqrt{-1} x_{\sigma 2}+x_{\sigma 3}\right)=\left(\left|b_{\sigma+1, \sigma}\right|^{2}-\left|b_{\sigma, \sigma+1}\right|^{2}, b_{\sigma, \sigma+1} b_{\sigma+1, \sigma}\right)$ as $\S 4.3$. By using the new coordinate $\left(x_{\sigma 1}, x_{\sigma 2}, x_{\sigma 3}, \theta_{\sigma}\right)$, the above equations are written as $t_{k}^{\sigma R}=x_{\sigma k}=t_{k}^{\sigma+1 L}$, and the metric is given by

$$
d s^{2}=\sum_{\sigma}\left\{\left(l_{\sigma}+l_{\sigma+1}+\frac{1}{\left|x_{\sigma}\right|}\right) d x_{\sigma}^{2}+\left(l_{\sigma}+l_{\sigma+1}+\frac{1}{\left|x_{\sigma}\right|}\right)^{-1}\left(d \psi_{\sigma}+\omega_{\sigma}\right)^{2}\right\} .
$$

Then the equations at $s_{\sigma}=0$ are

$$
-x_{\sigma k}+x_{\sigma+1 k}=-\zeta_{\sigma+1 k} .
$$

Put $x:=x_{0}=x_{1}+\zeta_{1}=x_{2}+\left(\zeta_{1}+\zeta_{2}\right)=\cdots=x_{\sigma}+\sum_{k=1}^{\sigma} \zeta_{k}$, and $\tilde{\zeta}_{\sigma}=\sum_{k=1}^{\sigma} \zeta_{k}$. Then we can write $x_{\sigma}=x-\tilde{\zeta}_{\sigma}$. Note that $\tilde{\zeta}_{0}=\sum_{k=1}^{n+1=0} \zeta_{k}=0$ by Remark 6.2. Hence we have

$$
d s^{2}=\left(2|\vec{l}|+\sum_{\sigma} \frac{1}{\left|x-\bar{\zeta}_{\sigma}\right|}\right) d x^{2}+\left(2|\vec{l}|+\sum_{\sigma} \frac{1}{\left|x-\bar{\zeta}_{\sigma}\right|}\right)^{-1}\left(\sum_{\sigma} d \psi_{\sigma}+\omega_{\sigma}\right)^{2} .
$$

This metric is a so-called multi-Taub-NUT metric. Summarising this section, we have the following theorem: 
Theorem 6.6. When $\zeta=0$, we have $\mathcal{M}_{0}^{A_{n}}(\vec{l}) \cong T N / A_{n}$ as hyper-Kähler manifolds. In general, $\mathcal{M}_{\zeta}^{A_{n}}(\vec{l})$ is a multi-Taub-NUT space and an ALF space.

Proof. The metric of $\mathcal{M}_{0}^{A_{n}}(\vec{l})$ depends only on $|\vec{l}|$. So it is enough to consider the case which all the $l_{\sigma}$ is equal, then the first assertion holds.

\section{7. $D_{n}$-type bow varieties}

In this section we consider the case of $D_{n}$ in the same way as $\S 6$.

7.1. $D_{n}$-type bow

We consider the following diagram $(n \geq 2)$ :

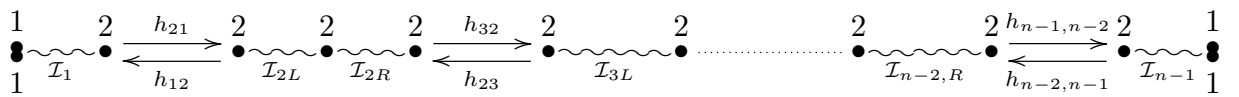

where $\mathcal{I}_{1}=\left\{0 \leq s_{1} \leq l_{1}\right\}, \mathcal{I}_{n-1}=\left\{-l_{n-1} \leq s_{n-1} \leq 0\right\}, \mathcal{I}_{\sigma L}=\left\{-l_{\sigma} \leq s_{\sigma} \leq 0\right\}$ and $\mathcal{I}_{\sigma R}=\left\{0 \leq s_{\sigma} \leq l_{\sigma}\right\}$. For $\vec{l}=\left(l_{1}, \cdots, l_{n-1}\right)$, we assume $|\vec{l}|:=l_{1}+l_{n-1}+\sum_{\sigma=2}^{n-2} 2 l_{\sigma}>0$. Put $\Omega=\left\{h_{\sigma+1, \sigma}\right\}$ and $\bar{\Omega}=\left\{h_{\sigma, \sigma+1}\right\}$.

We give rank-2 trivial hermitian vector bundles $\pi_{\sigma}: V_{\sigma} \rightarrow \mathcal{I}_{\sigma}$. In the same way as $\S 4.1 .$, let us define complex vector spaces $\mathbb{M}_{\Omega}^{D_{n}}, \mathbb{M}_{\Omega}^{D_{n}}$ and $\mathbb{M}^{D_{n}}$ respectively by

$$
\begin{aligned}
\mathbb{M}_{\Omega}^{D_{n}} & :=\bigoplus_{h \in \Omega} \operatorname{Hom}\left(V(o(h)), V(i(h)) \cong \bigoplus_{\sigma}\left\{B_{\sigma+1, \sigma} \in M(2,2 ; \mathbb{C})\right\},\right. \\
\mathbb{M}_{\bar{\Omega}}^{D_{n}} & :=\bigoplus_{h \in \bar{\Omega}} \operatorname{Hom}\left(V(o(h)), V(i(h)) \cong \bigoplus_{\sigma}\left\{B_{\sigma, \sigma+1} \in M(2,2 ; \mathbb{C})\right\},\right. \\
\mathbb{M}^{D_{n}}(\vec{l}) & :=\mathbb{M}_{\Omega}^{D_{n}} \oplus \mathbb{M}_{\bar{\Omega}}^{D_{n}} \oplus \bigoplus_{\sigma=2}^{n-1} \mathcal{H}_{\sigma L} \oplus \bigoplus_{\sigma=1}^{n-2} \mathcal{H}_{\sigma R} .
\end{aligned}
$$

When $l_{\sigma}=0$ for some $\sigma$, we treat $\mathbb{M}^{D_{n}}(\vec{l})$ in the same way as the case of the $A_{n}$-type, that is, we take the limit $l_{\sigma} \rightarrow 0$.

A group $\mathcal{G}^{D_{n}}=\mathcal{G}_{1} \times \mathcal{G}_{n-1} \times \prod_{\sigma \neq 1, n-1} \mathcal{G}_{\sigma}$ acts on $\mathbb{M}^{D_{n}}$, where

$$
\begin{aligned}
\mathcal{G}_{1} & =\left\{g_{1} \in L_{2}^{2}\left(\mathcal{I}_{1}, U(2)\right) \mid g_{1}(0) \in U(1) \times U(1) \subset U(2) \text { (diagonal) }\right\}, \\
\mathcal{G}_{n-1} & =\left\{g_{n-1} \in L_{2}^{2}\left(\mathcal{I}_{n-1}, U(2)\right) \mid g_{n-1}(0) \in U(1) \times U(1) \subset U(2) \text { (diagonal) }\right\}, \\
\mathcal{G}_{\sigma} & =\left\{g_{\sigma}=\left(g_{\sigma L}, g_{\sigma R}\right) \in L_{2}^{2}\left(\mathcal{I}_{\sigma L}, U(2)\right) \times L_{2}^{2}\left(\mathcal{I}_{\sigma L}, U(2)\right) \mid g_{\sigma L}(0)=g_{\sigma R}(0)\right\} .
\end{aligned}
$$

We have a hyper-Kähler moment map $\mu$ and the explicit form is as follows:

$$
\mu_{\mathbb{R}}= \begin{cases}\frac{-\sqrt{-1}}{2}\left(B_{\sigma, \sigma-1} B_{\sigma, \sigma-1}^{*}-B_{\sigma-1, \sigma}^{*} B_{\sigma-1, \sigma}\right)+T_{1}^{\sigma L}\left(-l_{\sigma}\right) & \text { at } s_{\sigma}=-l_{\sigma} \\ \frac{d}{d s} T_{1}^{\sigma A}+\left[T_{0}^{\sigma A}, T_{1}^{\sigma A}\right]+\left[T_{2}^{\sigma A}, T_{3}^{\sigma A}\right] & \text { at } s_{\sigma} \in\left(-l_{\sigma}, 0\right),\left(0, l_{\sigma}\right) \\ \frac{-\sqrt{-1}}{2}\left(B_{\sigma, \sigma+1} B_{\sigma, \sigma+1}^{*}-B_{\sigma+1, \sigma}^{*} B_{\sigma+1, \sigma}\right)-T_{1}^{\sigma R}\left(l_{\sigma}\right) & \text { at } s_{\sigma}=l_{\sigma} \\ -T^{\sigma L}(0)+T^{\sigma R}(0) & \text { at } s_{\sigma}=0, \text { for } \sigma \neq 1, n-1 \\ \pm T_{1}^{\sigma A}(0)^{1,1}, \pm T_{1}^{\sigma A}(0)^{2,2} & \text { at } s_{\sigma}=0, \text { for }\left(s_{\sigma}, A, \pm\right)=\left(s_{1}, R,+\right),\left(s_{n-1}, L,-\right),\end{cases}
$$


42

\section{YUUYA TAKAYAMA}

$$
\mu_{\mathbb{C}}=\left\{\begin{array}{lc}
B_{\sigma, \sigma-1} B_{\sigma-1, \sigma}+\left(T_{2}^{\sigma L}+\sqrt{-1} T_{3}^{\sigma L}\right)\left(-l_{\sigma}\right) & \text { at } s_{\sigma}=-l_{\sigma} \\
\frac{d}{d s}\left(T_{2}^{\sigma A}+\sqrt{-1} T_{3}^{\sigma A}\right)+\left[T_{0}^{\sigma A}+\sqrt{-1} T_{1}^{\sigma A}, T_{2}^{\sigma A}+\sqrt{-1} T_{3}^{\sigma A}\right] & \text { at } s_{\sigma} \in\left(-l_{\sigma}, 0\right),\left(0, l_{\sigma}\right) \\
-B_{\sigma, \sigma+1} B_{\sigma+1, \sigma}-\left(T_{2}^{\sigma}+\sqrt{-1} T_{3}^{\sigma}\right)\left(l_{\sigma}\right) & \text { at } s_{\sigma}=l_{\sigma} \\
-\left(T_{2}^{\sigma L}+\sqrt{-1} T_{3}^{\sigma L}\right)(0)+\left(T_{2}^{\sigma R}+\sqrt{-1} T_{3}^{\sigma L}\right)(0) & \text { at } s_{\sigma}=0, \text { for } \sigma \neq 1, n-1 \\
\pm\left(T_{2}^{\sigma A}+\sqrt{-1} T_{3}^{\sigma A}\right)(0)^{1,1}, \pm\left(T_{2}^{\sigma A}+\sqrt{-1} T_{3}^{\sigma A}\right)(0)^{2,2} & \text { at } s_{\sigma}=0, \text { for }\left(s_{\sigma}, A, \pm\right)=\left(s_{1}, R,+\right),\left(s_{n-1}, L,-\right) .
\end{array}\right.
$$

Put $Z^{D_{n}}=\left\{\zeta=\left(\zeta_{\sigma}\right) \in\left(\operatorname{Lie} U(2)^{n-1}\right)^{*} \otimes(\mathbb{R} \oplus \mathbb{C}) \mid \operatorname{Ad}_{g}^{*}(\zeta)=\zeta\right.$ for all $\left.g \in \mathcal{G}^{D_{n}}\right\}$. Choose an element $\zeta=\left(\zeta_{\mathbb{R}}, \zeta_{\mathbb{C}}\right) \in Z^{D_{n}}$, and define a hyper-Kähler quotient $\mathcal{M}_{\zeta}^{D_{n}}$ of $\mathbb{M}^{D_{n}}$ by $\mathcal{G}^{D_{n}}$ as follows:

$$
\mathcal{M}_{\zeta}^{D_{n}}(\vec{l}):=\left\{(B, T) \in \mathbb{M}^{D_{n}} \mid \mu(B, T)=\zeta \delta_{s_{\sigma}=0}\right\} / \mathcal{G}^{D_{n}} .
$$

We call this hyper-Kähler manifold a $D_{n}$-type bow variety.

REMARK 7.2. (i) In the case of $\vec{l}=(0,1,0, \cdots, 0)$, the hyper-Kähler manifold coincides with what Dancer constructed in $[\mathbf{D a}]$.

(ii) Put $\mathcal{G}_{\star 0}=\left\{g \in L_{2}^{2}(\mathcal{I}, U(2)) \mid g(l)=0, g(0) \in U(1) \times U(1) \subset U(2)\right.$ (diagonal) $\}$. A hyper-Kähler quotient of $\mathcal{H}$ by $\mathcal{G}_{\star 0}$ is holomorphic symplectomorphic to the cotangent bundle of $G L(n, \mathbb{C}) / \mathbb{C}^{*} \times \mathbb{C}^{*}$. This follows from $[\mathbf{D S}]$ and is considered as a generalisation of Kronheimer's theorem.

(iii) About the ends of the above diagram, one may think that (as appearing in [C2]) it is more natural to replace
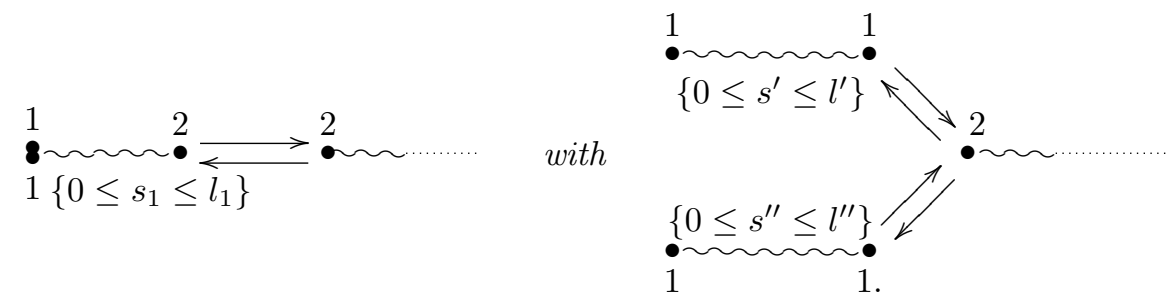

But in fact the former diagram contains the latter diagram.

First we consider the hyper-Kähler quotient $\mu^{-1}(\zeta) / \mathcal{G}^{D_{n}}$ of the latter diagram. Then we have

$$
\left\{\begin{array} { l | l } 
{ T _ { 1 } ^ { 1 } ( 0 ) = \zeta _ { \mathbb { R } } ^ { 1 } } & { ( T _ { 2 } ^ { 1 } + \sqrt { - 1 } T _ { 3 } ^ { 1 } ) ( 0 ) = \zeta _ { \mathbb { C } } ^ { 1 } } \\
{ T _ { 1 } ^ { 2 } ( 0 ) = \zeta _ { \mathbb { R } } ^ { 2 } } & { \frac { d } { d s } T _ { 1 } ^ { 1 } = 0 } \\
{ \frac { d } { d s } T _ { 1 } ^ { 2 } = 0 } & { ( T _ { 2 } ^ { 2 } + \sqrt { - 1 } T _ { 3 } ^ { 2 } ) ( 0 ) = \zeta _ { \mathbb { C } } ^ { 2 } } \\
{ \frac { - \sqrt { - 1 } } { 2 } ( B _ { 0 2 } B _ { 0 2 } ^ { * } - B _ { 2 0 } ^ { * } B _ { 2 0 } ) - T _ { 1 } ^ { 1 } ( l ^ { \prime } ) = 0 } \\
{ \frac { - \sqrt { - 1 } } { 2 } ( B _ { 1 2 } B _ { 1 2 } ^ { * } - B _ { 2 1 } ^ { * } B _ { 2 1 } ) - T _ { 1 } ^ { 2 } ( l ^ { \prime \prime } ) = 0 , }
\end{array} \quad \left\{\begin{array}{l}
\frac{d}{d s}\left(T_{2}^{2}+\sqrt{-1} T_{3}^{2}\right)=0 \\
-B_{02} B_{20}-\left(T_{2}^{1}+\sqrt{-1} T_{3}^{1}\right)\left(l^{\prime}\right)=0 \\
-B_{12} B_{21}-\left(T_{2}^{2}+\sqrt{-1} T_{3}^{2}\right)\left(l^{\prime \prime}\right)=0,
\end{array}\right.\right.
$$

for $T_{k}^{1}, T_{k}^{2} \in L_{1}^{2}(\mathcal{I}, \sqrt{-1} \mathbb{R})$. And we get

$$
\left\{\begin{array} { l } 
{ \frac { - \sqrt { - 1 } } { 2 } ( B _ { 0 2 } B _ { 0 2 } ^ { * } - B _ { 2 0 } ^ { * } B _ { 2 0 } ) = \zeta _ { \mathbb { R } } ^ { 1 } } \\
{ \frac { - \sqrt { - 1 } } { 2 } ( B _ { 1 2 } B _ { 1 2 } ^ { * } - B _ { 2 1 } ^ { * } B _ { 2 1 } ) = \zeta _ { \mathbb { R } } ^ { 2 } , }
\end{array} \quad \left\{\begin{array}{l}
-B_{02} B_{20}=\zeta_{\mathbb{C}}^{1} \\
-B_{12} B_{21}=\zeta_{\mathbb{C}}^{2}
\end{array}\right.\right.
$$

These equations also appear in the case of $l_{1}=0$ at the former diagram. In other words, 
even if we take $l^{\prime}, l^{\prime \prime} \neq 0$ at the latter diagram, the hyper-Kähler quotient $\mu^{-1}(\zeta) / \mathcal{G}^{D_{n}}$ does not depend on $l^{\prime}$ and $l^{\prime \prime}$.

On the other hand, from the former diagram we get the hyper-Kähler quotient depending on $l_{1}$ (see also Theorem 7.6).

7·2. General properties of $D_{n}$-type bow varieties

We can make the same arguments as the case of $\S 4.2$ and $\S 6.2$.

Proposition 7.3. The $D_{n}$-type bow variety $\mathcal{M}_{\zeta}^{D_{n}}$ is algebraic.

Proposition 7.4. As algebraic varieties, $\mathcal{M}_{\zeta}^{D_{n}}$ is isomorphic to a $D_{n}$-type quiver variety constructed by Kronheimer $[\mathbf{K r 2}]$ for $n \geq 3$.

It is well-known that $\mathbb{C}^{2} / D_{n}$ is isomorphic to an affine variety $\left\{(x, y, z) \in \mathbb{C}^{3}\right.$ | $\left.x^{2}-z y^{2}=z^{n-1}\right\}$ for $n \geq 3$. Combining this fact with Proposition 7.4, we have the isomorphism $\mathcal{M}_{0}^{D_{n}} \cong\left\{(x, y, z) \in \mathbb{C}^{3} \mid x^{2}-z y^{2}=z^{n-1}\right\}$ for $n \geq 3$. In fact for $n=2$, this is true $[\mathbf{D a}]$.

Proposition 7.5. $\mathcal{M}_{0}^{D_{2}}$ is isomorphic to $\left\{(x, y, z) \in \mathbb{C}^{3} \mid x^{2}-z y^{2}=z\right\}$.

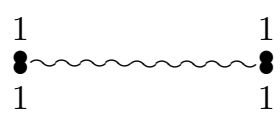

$D_{2}$-type bow.

Proof. We sketch the proof by Dancer. We consider $\mathcal{M}_{0}^{D_{2}}$ as an affine algebro-geometric quotient.

It is clear that the quotient of $G L(2) \times \mathfrak{g l}(2)$ by $(U(1) \times U(1)) \times(U(1) \times U(1))$ coincides with the quotient of $S L(2) \times \mathfrak{s l}(2)$ by $\mathbb{C}^{*} \times \mathbb{C}^{*}$. Here $\mathbb{C}^{*} \times \mathbb{C}^{*} \ni(a, b)$ acts as

$$
(a, b) \cdot(u, \eta):=\left(\left(\begin{array}{cc}
b & 0 \\
0 & b^{-1}
\end{array}\right) u\left(\begin{array}{cc}
a & 0 \\
0 & a^{-1}
\end{array}\right)^{-1},\left(\begin{array}{cc}
a & 0 \\
0 & a^{-1}
\end{array}\right) \eta\left(\begin{array}{cc}
a & 0 \\
0 & a^{-1}
\end{array}\right)^{-1}\right), \quad u \in S L(2), \eta \in \mathfrak{s l}(2) .
$$

Then the moment map is given by

$$
\mu_{\mathbb{C}}=\left\{\begin{array}{l}
\eta^{1,1}-\eta^{2,2} \\
\left(u \eta u^{-1}\right)^{1,1}-\left(u \eta u^{-1}\right)^{2,2} .
\end{array}\right.
$$

Therefore we consider $\mu_{\mathbb{C}}^{-1}(0) / \mathbb{C}^{*} \times \mathbb{C}^{*}$. Put $u=\left(\begin{array}{cc}x & y \\ z & w\end{array}\right), \eta=\left(\begin{array}{ll}0 & s \\ t & 0\end{array}\right)$. Since $u \in S L(2)$, we have $x w-y z=1$. And by $\mu_{\mathbb{C}}=0$, we get $t y w-s x z=0$. On the other hand $\mathbb{C}^{*} \times \mathbb{C}^{*}$ invariant polynomials are generated by $\{s t, s x z, t y w, x w, y z\}$. Put $X=s x z, \sqrt{-1} Y=$ $2 x w-1, Z=-\frac{s t}{4}$. By using the above equations, we have

$$
\begin{aligned}
X^{2} & =\operatorname{stxyzw} \\
& =-4 Z x w(x w-1)=-Z\left(-Y^{2}-1\right) .
\end{aligned}
$$

Thus $\mathcal{M}_{0}^{D_{2}} \cong\left\{(X, Y, Z) \in \mathbb{C}^{3} \mid X^{2}-Z Y^{2}=Z\right\}$ holds.

7·3. Metrics of $D_{n}$-type bow varieties

Let us consider the metric of $\mathcal{M}_{0}^{D_{n}}$.

Theorem 7.6. For $n \geq 3$, the $D_{n}$-type bow variety $\mathcal{M}_{0}^{D_{n}}(\vec{l})$ is isomorphic to $T N / D_{n}$ as hyper-Kähler manifolds. 


\section{YUUYA TAKAYAMA}

Proof. As we proved in $\S 5$, the $D_{n}$-type bow variety $\mathcal{M}_{0}^{D_{n}}$ is isomorphic to TN/ $D_{n}$ when $l_{1}=l_{2}=l_{n-2}=l_{n-1}$ and the morphism is given by the $\mathcal{G}$-action. TN/ $D_{n}$ is described by the explicit coordinate, and the coordinate does not depend on the length $l$, so for any $l_{1}, \cdots, l_{n-1}$ we can take the same coordinate. Thus all the $D_{n}$-type bow varieties with $\zeta=0$ are biholomorphic to each other and their metric depend only on $|\vec{l}|$. So, taking $l_{0}:=\frac{1}{2 n-4}|\vec{l}|$, we have

$$
\mathcal{M}_{0}^{D_{n}}\left(l_{1}, l_{2}, \cdots, l_{n-2}, l_{n-1}\right) \cong \mathcal{M}_{0}^{D_{n}}\left(l_{0}, l_{0}, \cdots, l_{0}, l_{0}\right) \cong \mathrm{TN}\left(l_{0}\right) / D_{n},
$$

as hyper-Kähler manifolds.

Corollary 7.7. For $n \geq 3$, the hyper-Kähler 4-manifold constructed by Dancer [Da] is $A L F$ when $\zeta=0$.

By Remark 5.7, we have the following proposition.

Proposition 7.8. $\mathcal{M}_{0}^{A_{3}}$ and $\mathcal{M}_{0}^{D_{3}}$ are isomorphic as algebraic varieties, but are not as hyper-Kähler manifolds.

REMARK 7.9. It is well-known that a $D_{n}$-type ALF space is obtained by dividing ( $2 n-$ 3)-centred multi-Taub-NUT space by $\iota$. By using our notation, this is written as follows.

Take $\vec{l}=\left(l_{1}, \cdots, l_{n-1}\right)$ and $\zeta=\left(\zeta_{1}, \cdots, \zeta_{n-1}\right) \in Z^{D_{n}}$ as $\zeta_{1}=c_{1} \mathrm{id}_{2}, \zeta_{n-1}=c_{n-1} \mathrm{id}_{2}$. Since for $\sigma \neq 1, n-1$ we also have $\zeta_{\sigma}=c_{\sigma} \mathrm{id}_{2}$, we get $\sum_{\sigma=1}^{n-1} c_{\sigma}=0$. This implies $\mathcal{M}_{\zeta}^{D_{n}}$ has an $A_{1}$-type singular point.

On the other hand, we construct an $A_{2 n-3}$-type bow variety with

$$
\begin{aligned}
& \vec{l}_{0}=\left(l_{1}, l_{2}, \cdots, l_{n-2}, l_{n-1}, l_{n-2}, \cdots, l_{2}\right), \\
& \zeta_{0}=\left(2 c_{1}, c_{2}, \cdots, c_{n-2}, 2 c_{n-1}, c_{n-2}, \cdots, c_{2}\right) .
\end{aligned}
$$

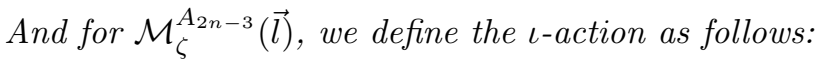

$$
\begin{aligned}
\left(u_{0}^{L}, \eta_{0}^{L}, u_{0}^{R}, \eta_{0}^{R}\right) & \mapsto\left(u_{0}^{R},-\eta_{0}^{R}, u_{0}^{L},-\eta_{0}^{L}\right), \\
\left(b_{\sigma, \sigma+1}, b_{\sigma+1, \sigma}\right) & \mapsto\left(-b_{2 n-2-\sigma, 2 n-3-\sigma}, b_{2 n-3-\sigma, 2 n-2-\sigma}\right), \\
\left(u_{\sigma}^{L}, \eta_{\sigma}^{L}, u_{\sigma}^{R}, \eta_{\sigma}^{R}\right) & \mapsto\left(u_{2 n-4-\sigma}^{R},-\eta_{2 n-4-\sigma}^{R}, u_{2 n-4-\sigma}^{L},-\eta_{2 n-4-\sigma}^{L}\right), \\
\left(u_{n-2}^{L}, \eta_{n-2}^{L}, u_{n-2}^{R}, \eta_{n-2}^{R}\right) & \mapsto\left(u_{n-2}^{R},-\eta_{n-2}^{R}, u_{n-2}^{L},-\eta_{n-2}^{L}\right) .
\end{aligned}
$$

Then for these $\vec{l}, \zeta$ and $\iota$, we have $\mathcal{M}_{\zeta}^{D_{n}}(\vec{l}) \cong \mathcal{M}_{\zeta_{0}}^{A_{2 n-3}}\left(\vec{l}_{0}\right) / \iota$.

All $D_{n}$-type bow varieties which we discussed in this subsection are ALF spaces and have singular points. According to these examples, we can conjecture that smooth $D_{n^{-}}$ type bow varieties are also ALF spaces and their metric depend not on each $l_{\sigma}$ but on the sum $|\vec{l}|$. But the author could not know how to prove these conjectures.

Acknowledgements. This work was prepared under the supervision of Professor Hiraku Nakajima, to whom the author is particularly grateful for all the help and advice he has received. And the author gratefully acknowledges valuable discussion of this work with Professor Sergey Cherkis.

\section{REFERENCES}

[Bi] R. Bielawski. Hyperkähler structure and group actions. J. Lond. Math. Soc. (2). 55 (1997), 400-414. 
[Br] J. BRYAN. Symplectic geometry and the relative Donaldson invariants of $\overline{\mathbb{C P}}^{2}$. Forum. Math. 9 (1997), 325-365.

[C1] S. Cherkis. Instantons on the Taub-NUT space. Adv. Theor. Math. Phys. 14 (2010), 609-641.

[C2] S. CheRKIs. Instantons on gravitons. Commun. Math. Phys. 306 (2011), 449-483.

[Da] A. DAncer. Dihedral singularities and gravitational instantons. J. Geom. Phys. 12 (1993), 77-91.

[DS] A. Dancer And A. Swann. Hyper-Kähler metrics associated to compact Lie groups. Math. Proc. Camb. Phil. Soc. 120 (1996), 61-69.

[Do] S. Donaldson. Nahm's equations and the classification of monopoles. Commun. Math. Phys. 96 (1984), 387-40\%.

[GN] T. Gocho AND H. NAKAJima. Einstein-Hermitian connections on hyper-Kähler quotients. J. Math. Soc. 44-1 (1992), 43-51.

[HKLR] N. Hitchin, A. Karlhede, U. Lindström And M. RoceK. Hyper-kähler metrics and supersymmetry. Commun. Math. Phys. 108 (1987), 535-589.

[Kin] A. KInG. Moduli of representations of finite dimensional algebras. Quarterly J. of Math. 45 (1994), 515-530.

[Ki] F. KIRWAN. Cohomology of quotients in symplectic and algebraic geometry. (Princeton University Press, 1984).

[Kr1] P. KRonheImer. A hyper-kähler structure on the cotangent bundle of a complex Lie group. arXiv:math/0409253v1.

[Kr2] P. Kronheimer. The construction of ALE spaces as hyper-Kähler quotients. J. Diff. Geom. 29 (1989), 665-683.

[KN] P. KRonheimer and H. NAKajima. Yang-Mills instantons on ALE Gravitational instantons. Math. Ann. 288-2 (1990), 263-30\%.

[M] V. MinerBe. On some asymptotically flat manifolds with non maximal volume growth. arXiv:0709.1084v1.

[MFK] D. Mumford, J. Fogarty and F. Kirwan. Geometric invariant theory. (SpringerVerlag, 1994).

[Na1] H. NAKAJImA. Instantons on ALE spaces, quiver varieties, and Kac-Moody algebras. Duke Math. J. 76-2 (1994), 365-416.

[Na2] H. NAKAJima. Lectures on Hilbert Schemes of Points on Surfaces. (American Mathematical Society, 1999).

[Ne] A. NeEman. The topology of quotient varieties. Ann. of Math. 103-2 (1985), 419459.

[V] V. VAradarajan. Lie groups, Lie algebras, and their representations. (Prentice-Hall, inc). 